University of Tennessee Health Science Center UTHSC Digital Commons

\title{
The Effect of Timbre and Vibrato on Vocal Pitch Matching Accuracy
}

Sirisha Duvvuru

University of Tennessee Health Science Center

Follow this and additional works at: https://dc.uthsc.edu/dissertations

Part of the Speech and Hearing Science Commons, and the Speech Pathology and Audiology Commons

\section{Recommended Citation}

Duvvuru, Sirisha, "The Effect of Timbre and Vibrato on Vocal Pitch Matching Accuracy" (2012). Theses and Dissertations (ETD). Paper 71. http://dx.doi.org/10.21007/etd.cghs.2012.0076.

This Dissertation is brought to you for free and open access by the College of Graduate Health Sciences at UTHSC Digital Commons. It has been accepted for inclusion in Theses and Dissertations (ETD) by an authorized administrator of UTHSC Digital Commons. For more information, please contact jwelch30@uthsc.edu. 


\title{
The Effect of Timbre and Vibrato on Vocal Pitch Matching Accuracy
}

\begin{abstract}
Research has shown that singers are better able to match pitch when the target stimulus has a timbre close to their own voice. This study seeks to answer the following questions: 1 . Do classically trained female singers more accurately match pitch when the target stimulus is more similar to their own timbre? 2. Does the ability to match pitch vary with increasing pitch? 3. Does the ability to match pitch differ depending on whether the target stimulus is produced with or without vibrato? 4. Are mezzo sopranos less accurate than sopranos? Stimuli: Source signals were synthesized with a source slope of $-12 \mathrm{~dB} /$ octave using vibrato and without vibrato at each of the frequencies, C4, B4 and F5. These source signals were filtered using 5 formant patterns (A-E) of vowel /a/ constituting a total of 30 stimuli ( 5 formant patterns $* 3$ pitches $* 2$ vibrato conditions). Procedure: Ten sopranos and 10 mezzo-sopranos with at least 3 years of individual voice training were recruited from the University Of Tennessee School Of Music and the Knoxville Opera Company. Each singer attempted to vocally match the pitch of all 30 stimuli presented twice in a random order. Analysis and results: Pitch matching accuracy was measured in terms of the difference in cents between the target and the experimental productions at two locations, (1) prephonatory set (2) mid-point of the vowel. Accuracy of pitch matching was compared across vibrato and nonvibrato conditions. Results indicated that there was no significant effect of formant pattern on pitch matching accuracy. With increasing pitch from C4 to F5, pitch matching accuracy increased in mid-point of the vowel condition but not in pre-phonatory set condition. Mezzosopranos moved towards being in tune from pre-phonatory to mid-point of the vowel. However, sopranos at $\mathrm{C} 4$, sang closer to being in tune at pre-phonatory, but lowered the pitch at the midpoint of the vowel. Presence or absence of vibrato did not affect the pitch matching accuracy. However, the interesting finding of the study was that singers attempted to match the timbre of stimuli with vibrato. Results are discussed in terms of interactions between pitch and timbre from auditory perceptual as well as physiological point of view and how current theories of pitch perception relate to this phenomenon. Neither physiological nor auditory perceptual mechanisms provide complete explanations for the results obtained in the study. From a perceptual point of view, an interaction between pitch and timbre seems to be more complex, for spectral and temporal theories are limited in explaining these interactions. Also, possible explanations for the phenomenon of timbre matching are provided.
\end{abstract}

\section{Document Type}

Dissertation

Degree Name

Doctor of Philosophy (PhD)

Program

Speech and Hearing Science

Research Advisor

Mary L. Erickson, Ph.D.

\section{Keywords}

centroid, mezzo-soprano, pitch, soprano, timbre, vibrato

\section{Subject Categories}

Communication Sciences and Disorders | Medicine and Health Sciences | Speech and Hearing Science | Speech Pathology and Audiology 
The Effect of Timbre and Vibrato on Vocal Pitch Matching Accuracy

\author{
A Dissertation \\ Presented for \\ The Graduate Studies Council \\ The University of Tennessee \\ Health Science Center
}

In Partial Fulfillment

Of the Requirements for the Degree

Doctor of Philosophy

From The University of Tennessee

By

Sirisha Duvvuru

December 2012 
Copyright (C) 2012 by Sirisha Duvvuru. All rights reserved. 


\section{DEDICATION}

To my mom, Late. Shanthi, my dad, Sudhakar Duvvuru, my brother and sister in law, Suman and Spandana, and my grandmom, Adilakshmi Duvvuru. 


\section{ACKNOWLEDGEMENTS}

I would like to express my deepest gratitude to my advisor, Dr. Molly Erickson, for her guidance, caring, support, and providing me with an excellent learning experience for doing research. I would like to thank Dr. Kristen King, who always provided valuble suggestions and ideas for improving research in all my projects as well as the dissertation. I would also like to thank Dr. Mark Hedrick for his contribution to my works and a friendly support throughout. I would also like to thank Dr.Daniela Corbetta for actively involving and guiding my research and help me learn to collaborate with allied fields.

Special thanks to all the singers who participated in the study by volunteering and helping me finish my dissertation on time. I would also thank my co-workers, Sukhada vaidya and Daniela Oliveira who were always involved in sharing ideas and providing personal support. Iam extremely grateful to my family members, friends and all the loved ones for their encouragement. 


\begin{abstract}
Research has shown that singers are better able to match pitch when the target stimulus has a timbre close to their own voice. This study seeks to answer the following questions: 1 . Do classically trained female singers more accurately match pitch when the target stimulus is more similar to their own timbre? 2. Does the ability to match pitch vary with increasing pitch? 3 . Does the ability to match pitch differ depending on whether the target stimulus is produced with or without vibrato? 4. Are mezzo sopranos less accurate than sopranos?
\end{abstract}

Stimuli: Source signals were synthesized with a source slope of $-12 \mathrm{~dB} /$ octave using vibrato and without vibrato at each of the frequencies, C4, B4 and F5. These source signals were filtered using 5 formant patterns (A-E) of vowel /a/ constituting a total of 30 stimuli (5 formant patterns*3pitches*2 vibrato conditions).

Procedure: Ten sopranos and 10 mezzo-sopranos with at least 3 years of individual voice training were recruited from the University Of Tennessee School Of Music and the Knoxville Opera Company. Each singer attempted to vocally match the pitch of all 30 stimuli presented twice in a random order.

Analysis and results: Pitch matching accuracy was measured in terms of the difference in cents between the target and the experimental productions at two locations, (1) pre-phonatory set (2) mid-point of the vowel. Accuracy of pitch matching was compared across vibrato and non-vibrato conditions. Results indicated that there was no significant effect of formant pattern on pitch matching accuracy. With increasing pitch from $\mathrm{C} 4$ to $\mathrm{F} 5$, pitch matching accuracy increased in mid-point of the vowel condition but not in pre-phonatory set condition. Mezzo-sopranos moved towards being in tune from pre-phonatory to mid-point of the vowel. However, sopranos at C4, sang closer to being in tune at pre-phonatory, but lowered the pitch at the mid-point of the vowel. Presence or absence of vibrato did not affect the pitch matching accuracy. However, the interesting finding of the study was that singers attempted to match the timbre of stimuli with vibrato.

Results are discussed in terms of interactions between pitch and timbre from auditory perceptual as well as physiological point of view and how current theories of pitch perception relate to this phenomenon. Neither physiological nor auditory perceptual mechanisms provide complete explanations for the results obtained in the study. From a perceptual point of view, an interaction between pitch and timbre seems to be more complex, for spectral and temporal theories are limited in explaining these interactions. Also, possible explanations for the phenomenon of timbre matching are provided. 


\section{TABLE OF CONTENTS}

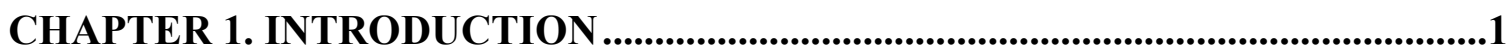

What Is Pitch and What Kind of Information Does It Carry ..........................................

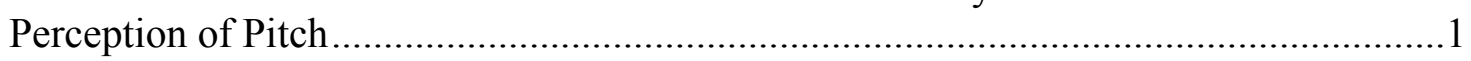

The Importance of Pitch Perception and Production .........................................................

The Role of Timbre in Pitch Perception and Its Relevance to the Present Study............2

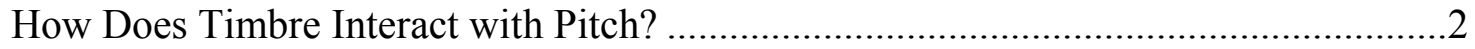

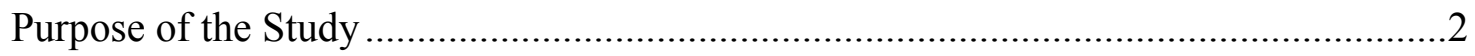

CHAPTER 2. REVIEW OF THE LITERATURE......................................................

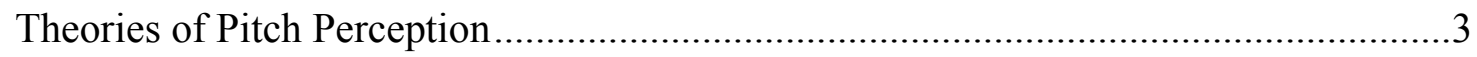

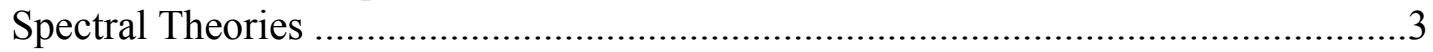

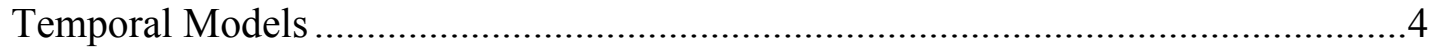

Spectral or Temporal Pitch Extraction.....................................................................

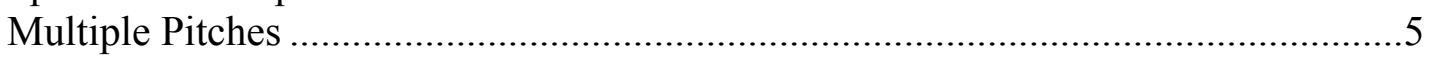

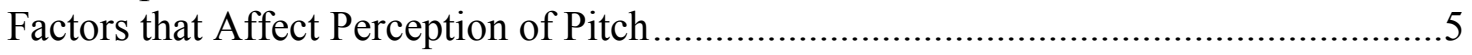

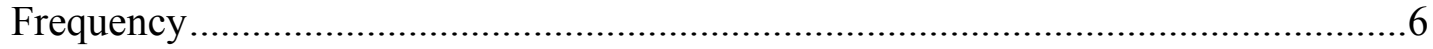

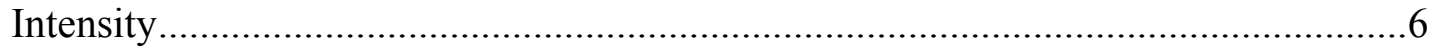

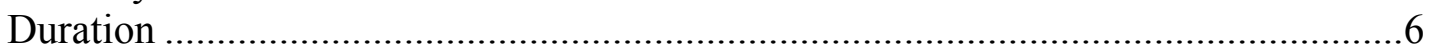

Timbre

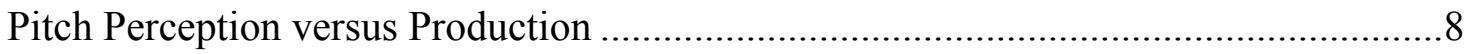

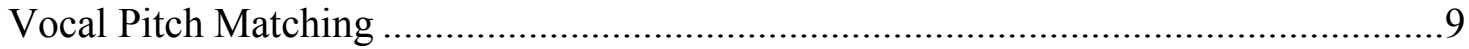

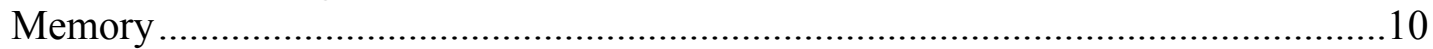

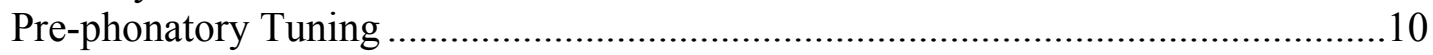

Auditory Feedback ……………………......................................................11

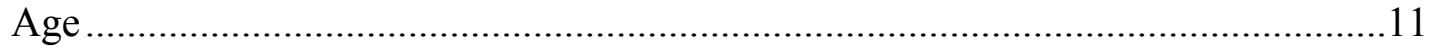

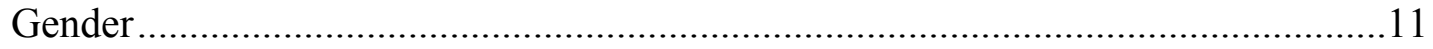

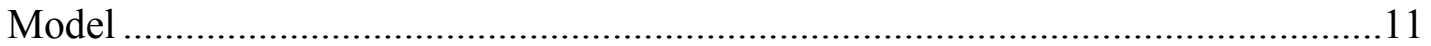

Spectral Composition and Voice Classification ………...............................................14

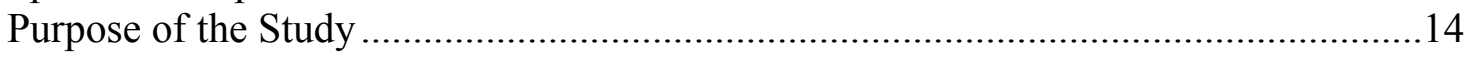

CHAPTER 3. METHOD .................................................................................................17

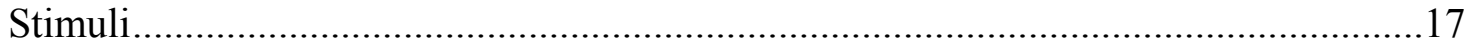

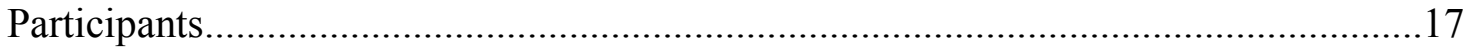

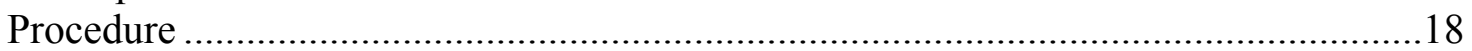

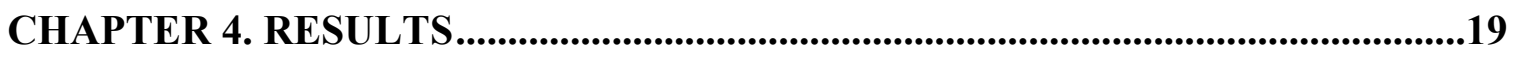

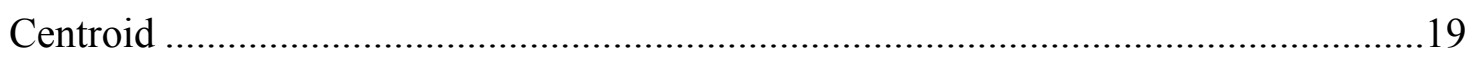

Omnibus Analysis.........................................................................................19

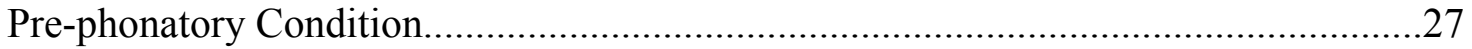

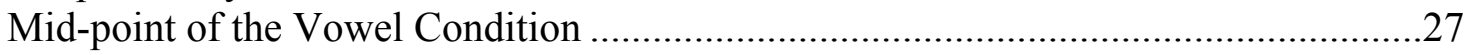

Difference between Pre-phonatory and Mid-point of the Vowel Condition ..................41 


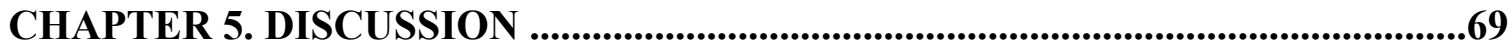

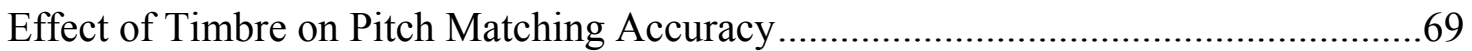

Effect of Target Pitch on the Pitch Matching Accuracy .................................................70

Effect of Vibrato on Pitch Matching Accuracy ..........................................................71

Effect of Category on Pitch Matching Accuracy ……….............................................73

The Effect of Target Timbre on Spectral Centroid.......................................................74

Limitations of the Study .....................................................................................

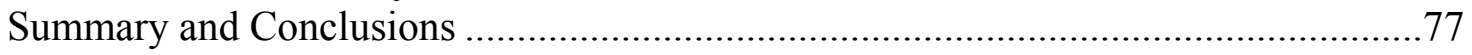

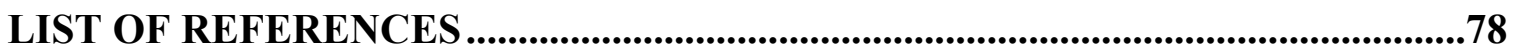

VITA 


\section{LIST OF TABLES}

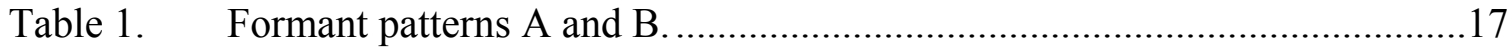

Table 2. Results of $2 \times 3 \times 5 \times 2$ ANOVA for mean centroid in semitones. ....................20

Table 3. Results of post-hoc ANOVA for mean centroid as a function of pitch and pattern in vibrato condition.

Table 4. Results of post-hoc ANOVA for mean centroid as a function of pitch and pattern in no-vibrato condition.

Table 5. Results of $2 \times 2 \times 2 \times 3 \times 5$ repeated measures ANOVA for difference in cents between the target stimuli and the experimental productions.

Table 6. Results of post-hoc ANOVA for mean difference in cents between target

stimuli and experimental productions at pitch $\mathrm{C} 4$.

Table 7. Results of post-hoc ANOVA for mean difference in cents between target stimuli and experimental productions at pitch B4.

Table 8. Results of post-hoc ANOVA for mean difference in cents between target stimuli and experimental productions at pitch F5.

Table 9. Results of $2 \times 3 \times 5 \times 2$ ANOVA for mean difference in cents between the target and the experimental productions in pre-phonatory condition.

Table 10. Results of $2 \times 3 \times 5 \times 2$ ANOVA for mean difference in cents between the target and the experimental productions in mid-point of the vowel condition.

Table 11. Results post-hoc ANOVA for mean difference in cents as a function of pitch and pattern in vibrato condition for the mid-point of the vowel.

Table 12. Results post-hoc ANOVA for mean difference in cents as a function of pitch and pattern in no-vibrato condition for the mid-point of the vowel....42

Table 13. Results of $2 \times 3 \times 5 \times 2$ ANOVA for mean difference in cents from prephonatory to mid-point of the vowel condition.

Table 14. Results post-hoc ANOVA for mean difference in cents from prephonatory to mid-point of the vowel as a function of pitch and pattern in vibrato condition.

Table 15. Results post-hoc ANOVA for mean difference in cents from prephonatory to mid-point of the vowel as a function of pitch and pattern in no-vibrato condition. 


\section{LIST OF FIGURES}

Figure 1. Hypothetical representation showing enhanced pitch matching ability from formant pattern A to formant pattern $\mathrm{E}$.

Figure 2. Overview of statistical analysis including Bonferroni corrections at each level of analysis

Figure 3. Mean centroid in semitones for mezzo-sopranos and sopranos as a function of formant pattern and vibrato at pitch $\mathrm{C} 4$.

Figure 4. Mean centroid in semitones for mezzo-sopranos and sopranos as a function of formant pattern and vibrato at pitch B4.

Figure 5. Mean centroid in semitones for mezzo-sopranos and sopranos as a function of formant pattern and vibrato at pitch F5.

Figure 6. Mean centroid in semitones as a function of formant pattern in vibrato condition.

Figure 7. Mean centroid in semitones as a function of formant pattern in novibrato condition.

Figure 8. Mean difference in cents between the target and the experimental productions as a function of formant pattern and vibrato at pitch $\mathrm{C} 4$ in pre-phonatory condition.

Figure 9. Mean difference in cents between the target and the experimental productions as a function of formant pattern and vibrato at pitch B4 in pre-phonatory condition.

Figure 10. Mean difference in cents between the target and the experimental productions as a function of formant pattern and vibrato at pitch F5 in pre-phonatory condition.

Figure 11. Mean difference in cents between the target and the experimental productions as a function of formant pattern and vibrato at pitch $\mathrm{C} 4$ in mid-point of the vowel condition.

Figure 12. Mean difference in cents between the target and the experimental productions as a function of formant pattern and vibrato at pitch B4 in mid-point of the vowel condition.

Figure 13. Mean difference in cents between the target and the experimental productions as a function of formant pattern and vibrato at pitch F5 in mid-point of the vowel condition. 
Figure 14. Mean difference in cents as a function of pitch $\mathrm{C} 4$ for mezzo-sopranos and sopranos in vibrato condition for the mid-point of the vowel.

Figure 15. Mean difference in cents as a function of pitch B4 for mezzo-sopranos and sopranos in vibrato condition for the mid-point of the vowel.

Figure 16. Mean difference in cents as a function of pitch F5 for mezzo-sopranos and sopranos in vibrato condition for the mid-point of the vowel.

Figure 17. Mean difference in cents as a function of pitch $\mathrm{C} 4$ for mezzo-sopranos and sopranos in no-vibrato condition for the mid-point of the vowel.

Figure 18. Mean difference in cents as a function of pitch B4 for mezzo-sopranos and sopranos in no-vibrato condition for the mid-point of the vowel.

Figure 19. Mean difference in cents as a function of pitch F5 for mezzo-sopranos and sopranos in no-vibrato condition for the mid-point of the vowel

Figure 20. Mean difference in cents as a function of pitch for vibrato and no-vibrato conditions for the mid-point of the vowel.

Figure 21. Mean difference in cents between pre-phonatory and mid-point of the vowel conditions as a function of formant pattern and vibrato at pitch C4.

Figure 22. Mean difference in cents between pre-phonatory and mid-point of the vowel conditions as a function of formant pattern and vibrato at pitch B4.

Figure 23. Mean difference in cents between pre-phonatory and mid-point of the vowel conditions as a function of formant pattern and vibrato at pitch F5.

Figure 24. Mean difference in cents between pre-phonatory and mid-point of vowel in vibrato condition at pitch $\mathrm{C} 4$.....

Figure 25. Mean difference in cents between pre-phonatory and mid-point of vowel in vibrato condition at pitch $\mathrm{B} 4$.

Figure 26. Mean difference in cents between pre-phonatory and mid-point of vowel in vibrato condition at pitch F5.

Figure 27. Mean difference in cents between pre-phonatory and mid-point of vowel in no-vibrato condition at pitch $\mathrm{C} 4$.

Figure 28. Mean difference in cents between pre-phonatory and mid-point of vowel in no-vibrato condition at pitch B4. 
Figure 29. Mean difference in cents between pre-phonatory and mid-point of vowel in no-vibrato condition at pitch F5.

Figure 30. Vector plot representing mean difference in cents from pre-phonatory phase to mid-point of the vowel in vibrato condition at pitch $\mathrm{C} 4$.

Figure 31. Vector plot representing mean difference in cents from pre-phonatory phase to mid-point of the vowel in vibrato condition at pitch B4.

Figure 32. Vector plot representing mean difference in cents from pre-phonatory phase to mid-point of the vowel in vibrato condition at pitch F5.

Figure 33. Vector plot representing mean difference in cents from pre-phonatory phase to mid-point of the vowel in no-vibrato condition at pitch $\mathrm{C} 4$.

Figure 34. Vector plot representing mean difference in cents from pre-phonatory phase to mid-point of the vowel in no-vibrato condition at pitch B4.

Figure 35. Vector plot representing mean difference in cents from pre-phonatory phase to mid-point of the vowel in no-vibrato condition at pitch F5.

Figure 36. Mean difference in cents between pre-phonatory and mid-point of the vowel as a function of pitch for vibrato and no-vibrato conditions.

Figure 37. Change in pitch matching accuracy as a function of target pitch for mezzo-sopranos and sopranos averaged across all patterns and vibrato conditions.

Figure 38. Change in pitch matching accuracy between pre-phonatory set to midpoint of the vowel averaged over all the pitches. 


\section{CHAPTER 1. INTRODUCTION}

\section{What Is Pitch and What Kind of Information Does It Carry}

The American National Standard Institute (ANSI, 1973) defines pitch as that attribute of auditory sensation in terms of which sounds may be ordered from low to high. Variations in pitch create a sense of melody and harmony in music and also carry the bulk of prosodic information in speech. Pitch is the perceptual interpretation of frequency. Typically, human hearing ranges from 20 to $20,000 \mathrm{~Hz}$, however, we have our greatest sensitivity to frequencies which lie within 500 to 2000 .

\section{Perception of Pitch}

The smallest detectable frequency difference between two tones is often referred to as the difference limen for frequency (DLF) (Gelfand, 1998). The difference limen can be measured by asking listeners to judge whether the second tone in a pair is higher/lower in pitch than the first. The difference limen is calculated as the frequency difference between tones for which subjects provide a specific percentage of correct responses, commonly $75 \%$. Using an adaptive procedure, the frequency difference between two tones is gradually reduced as the listener makes correct responses and increased as the listener makes incorrect responses. Alternately, the method of constant stimuli can also be used to measure DLF.

\section{The Importance of Pitch Perception and Production}

The accuracy of pitch perception is important for clinical management purposes and influences both the monitoring of one's fundamental frequency $\left(\mathrm{F}_{0}\right)$ during speaking and how accurately an individual maintains a desired pitch or volitionally changes pitch. Accurate perception of pitch precedes accurate production and therefore affects the ability to produce a desired $\mathrm{F}_{0}$, to produce prosodic changes in speaking, and to sing in tune.

Pitch perception is critical for ability to sing. Singers often have to discriminate and produce pitches accurately. A survey on 1000 music educators showed that pitch intonation was found to be the most important factor for determining singing ability followed by timbre and musicality (melodic connection between the notes) (Watts et al, 2003). It is logical to assume that pitch intonation is directly related to accuracy of pitch matching. 


\section{The Role of Timbre in Pitch Perception and Its Relevance to the Present Study}

There are several factors which affect vocal pitch matching ability such as age, gender, and vocal model, as well as stimulus properties such as frequency, intensity and timbre. Though there are a myriad of factors that influence pitch matching ability, the present study is being carried out to explore pitch matching ability as the function of timbre. Timbre is defined as that property of sound which makes two tones sound different, even though they have same loudness and pitch (ANSI, 1973). In the field of vocal pedagogy, timbre plays a crucial role as it forms a basis for voice classification in singers. Each singer has their own timbre which is a product of the laryngeal voice source and vocal tract resonances (standing wave frequencies of vocal tract). The hypothesis that singers with similar timbres fall into one common voice category led to the traditional classification of singing voices into bass, baritone tenor, alto, mezzo-soprano and soprano.

\section{How Does Timbre Interact with Pitch?}

From several studies in the literature (Crowder, 1989; Green, 1990; Platt and Racine, 1985; Watts and Hall, 2005), we know that listeners can resolve pitch quite well when the target model is a similar timbre to their own. However, when listeners need to make pitch judgments across the timbres, they may need to translate the target timbre into the timbre of their own voice (Crowder, 1989). The accuracy of pitch matching seems to be reduced in this case. One of the acoustic properties that has been shown to affect perception of pitch across different timbre is spectral composition, i.e., energy distribution in harmonics (Moore et.al, 1992; Patterson, 1990, Singh and Hirsh, 1992). Stimuli with greater energy in higher harmonics can shift pitch perception upwards (Worthy, 2000). According to Singh and Hirsh (1992), when the $F_{0}$ change is small, less than $2 \%$, spectral centroid change is the primary predictor of perceived pitch change. If this is true, in the case of singers who exhibit high pitch deviations (poor singers), timbre differences might have very little influence on the perception and production of pitch. However, in the case of singers whose pitch deviations are less than $2 \%$, it is possible the timbre differences between the model and the singer's own timbre might affect both the perception and production of pitch.

\section{Purpose of the Study}

Most studies in the current literature focus on the pitch perception and production abilities of poor singers (Demorest, 2001; Lévêque, Giovanni, \& Schön, 2011; Watts et al, 2005; Yarbrough et al, 1992). However, minor pitch perception and production problems can have a devastating effect on highly-trained professional singers who must compete against intense competition for jobs. The present investigation focuses on welltrained singers who are likely to produce small errors in pitch (either slightly "flat" or "sharp) to determine if the timbre of the vocal model influences their vocal pitch matching accuracy. 


\section{CHAPTER 2. REVIEW OF THE LITERATURE}

\section{Theories of Pitch Perception}

There are several theories that have been proposed that attempt to explain how human's perceive pitch. Some of the theories are based on spectral analysis where a complex sound is decomposed into individual sinusoidal components via the cochlea. Other theories are based on temporal analysis where fundamental frequency is extracted from time-based events.

\section{Spectral Theories}

Resonance-place theory. According to resonance-place theory (Helmholtz, 1954), the inner ear acts like a mechanical frequency analyzer, similar to a series of Helmholtz resonators, hence the name. Helmholtz characterized the filters of the cochlea as resonators each tuned to a single harmonic. These resonators then decompose complex waveforms into their component sine waves. He believed it was the perception of the frequency of the first harmonic, or fundamental, that produced the sensation of pitch. However, it is well known that pitch of a complex sound can be perceived even when energy at its fundamental frequency has been removed. This phenomenon, called the missing fundamental, cannot be explained by resonance-place theory. To address this shortcoming, Helmholtz suggested several processes whereby the fundamental frequency could be extracted: nonlinear distortion, unconscious interference, and the functioning of the cochlea as a system of string resonators. Experiments by Schouten (1938) and Licklider (1954) showed that removing or masking the distortion product at the fundamental frequency did not prevent the perception of pitch, suggesting that non-linear distortion did not fully account for the missing fundamental problem. Helmhotz's unconscious interference was a more likely proposal and foreshadows later pattern matching solutions.

The residue. Schouten (1940) developed "the residue" hypothesis to account for the perception of the missing fundamental. Originally, the residue pitch was thought to be due to extraction of the missing fundamental from beating of higher, unresolved harmonics. However, Ritsma (1967) and Plomp (1967) showed that resolved harmonics dominate pitch perception. For this reason, de Boer (1976) expanded Schouten's model to include all harmonics, resolved and unresolved, thereby implying that the residue is, or at least arises from, the entire sound, not part of the sound.

Pattern matching models. In general, pattern matching models are based on the idea that the human brain perceives pitch by extracting the fundamental frequency based on the pattern of component harmonics, even when the fundamental is missing. Pattern matching models assume that a first step in pitch perception is decomposition of a complex wave into its component harmonics, and therefore, these types of models fall into the category of spectral models. The second step in the process is to match the 
pattern of harmonics to best fitting template pattern that may either be pre-existing in memory (Terhardt, 1974) or may somehow be derived from processing in the early stages of the auditory system (Shamma \& Klein, 2000). The fundamental of the best fitting pattern is the fundamental frequency of the complex sound. Three of the best known pattern matching models were proposed by Goldstein (1973), Terhardt (1974), and Wightman (1973).

Goldstein (1973) proposed a probabilistic model in which the pattern is modeled as a series of harmonics that are transformed into Gaussian distributions in an attempt to represent noise present in the auditory system. A central processor then estimates the fundamental frequency using a maximum likelihood estimation of the harmonic sets providing the best match.

Wightman (1973) formalized a mathematical model called "the pattern transformation model" of pitch, wherein pitch perception is based on a sequence of transformations which produce different patterns of neural activity. Wightman used the term pattern to refer to a two dimensional distribution of neural activity, place and amplitude. Different places in the pattern represented individual or groups of neurons, and the amplitude indicated the activity of these neurons. In his model, the peripheral activity pattern undergoes a Fourier transformation to produce an autocorrelation function. Finally, pitch is derived from the peak in the transformed pattern using a pattern matching strategy.

Terhardt (1974a) suggested that the essential principle in explaining the phenomena of pitch perception is the distinction between spectral pitch and virtual pitch. He suggested that the pitch of a pure tone is derived from spectral decomposition and is therefore termed spectral pitch; however, the pitch of a complex tone is a virtual pitch because it does not depend on energy being present at the fundamental. Although these are two distinct kinds of pitch perception, both are derived from spectral cues. Virtual pitch is derived through a pattern matching process wherein the subharmonic structures of each harmonic are determined. The most frequently occurring subharmonic is perceived as the virtual pitch.

All the models described above depend on the spectral resolution of individual components in the stimulus, and therefore, fail to identify the pitch of complex tones whose harmonics are too close to be resolved and also fail when there is no well-defined structure in the stimulus such as the case of interrupted noise.

\section{Temporal Models}

Temporal models assume a time-domain mechanism which is event-based; i.e., it tries to detect the time interval between events. The most likely event would be the timing of neural firings that occur in response to vibration of basilar membrane. Typically, distinct patterns of neural firings occur only at particular locations along the basilar membrane. The vibrations create synchronous firing of the tonotopically 
organized auditory nerve fibers, a phenomenon called phase locking. Because of phase locking, time intervals between successive firings occur at approximate integral multiples of the period of the waveform. However, the relation between the neural firing and a particular phase is diminished in its precision at frequencies above approximately $5 \mathrm{kHz}$ (Johnson, 1980). Thus this theory cannot explain the ability to perceive pitches at frequencies higher than $5 \mathrm{kHz}$. Unlike Helmholtz's theory, temporal theory can explain the phenomena of the missing fundamental.

Extraction of a pitch period based on events is most accurately performed via autocorrelation analysis. Licklider (1951) was the first person to introduce a method of autocorrelation analyses in his duplex theory of pitch perception, which states that our auditory system employs both frequency (spectral) analyses and autocorrelation analyses (temporal) for sensation of pitch. Licklider proposed that after a frequency analysis is performed on the complex signal, the hair cells of the cochlea send the original signal to a series of neurons that in effect combine the original signal with a time-delayed signal, essentially producing an autocorrelation function at each frequency.

\section{Spectral or Temporal Pitch Extraction}

Much recent research has focused on the idea that in certain circumstances listeners may employ spectral pitch extraction via pattern matching, deriving the fundamental frequency from analysis of resolved partials; whereas in other situations, listeners may use temporal pitch extraction, deriving the fundamental frequency from autocorrelation of the phase-locked neuronal firing along the basal membrane (Grimault, Micheyl, Carylon, \& Collet, 2002; Terhardt, Stoll, \& Seewann, 1982).

\section{Multiple Pitches}

Narrowly tuned formants have been shown to induce the perception of a second pitch by greatly amplifying a single harmonic. This is the manner in which Tibetan "throat" singing is produced. In cases like this, the perception of the formant as a pitch is often said to be a spectral pitch, since it arises from the spectrum. However, de Cheviené (2005) points out that such a strong harmonic would be of sufficiently high amplitude that it would introduce a quasi-periodicity into the time-domain neural signal, suggesting that it may also be extracted through temporal means.

\section{Factors that Affect Perception of Pitch}

There are numerous factors that can influence the perception of pitch. Four of the main factors are (1) frequency, (2) intensity, (3) duration, and (4) timbre. 


\section{Frequency}

Frequency is rate of vibration per second. While there is a monotonic relationship between frequency and pitch, the relationship is neither linear nor logarithmic, although it is much closer to logarithmic. Generally a doubling of frequency results in the perception of an increase of one octave, or 12 semitones. This means that a specific change in frequency at lower frequencies results in a greater perceived change in pitch than the same change in frequency will at higher frequencies. But the relationship is more complex than just this logarithmic conversion. Just as the sone scale relates intensity and loudness, the complex relationship between pitch and frequency is depicted by Mel scale in which the unit of pitch is Mel (Stevens, 1975). Mel scale is the result of classic frequency halving/doubling experiments in which listeners were asked to adjust the frequency of complex tone until its pitch was twice or half of that of the test tone. From Stevens' data, it was found that halving/doubling the pitches is not equal to halving/doubling of frequency.

\section{Intensity}

Stevens (1935) studied the relationship of pitch and intensity and reported that increases in intensity result in a perception of increased pitch and decreases in intensity result in a perception of decreased pitch with the maximum pitch shifts on the order of $5 \%$ to $10 \%$. However, later studies showed much a smaller effect of intensity on pitch perception (Terhardt, 1974b; Verchure \& Van Meeteren, 1975).

\section{Duration}

The quality of a pure tone changes as a function of its duration. A sinusoid will sound like a click at very short durations, but as its duration is increased perception of pitch becomes clearer. The point where the perception changes from a click to a tone is dependent on frequency (Doughty \& Garner, 1947). They revealed three stages of pitch perception as a function of duration. In the first stage, a click is heard. In the second stage a click with some pitch character is heard. In the third stage a tone with definite pitch character is heard. It has been hypothesized that perception of pitch will be different for short versus long duration tones. Doughty and Garner (1948) presented listeners with a standard tone of $500 \mathrm{~ms}$ and comparison tones of six different durations : 6, 12, 25, 50, 100 , or $200 \mathrm{~ms}$ and instructed them to adjust the frequency of the comparison tone to that of the standard tone until they were perceived equal in pitch. The results of the study showed that very short tones (6 or $12 \mathrm{~ms}$ ) were heard lower in pitch when compared to a $50-\mathrm{ms}$ tone with the same frequency. This effect was most prominent at $250 \mathrm{~Hz}$ and did not affect tones with durations of $25 \mathrm{~ms}$ or longer. However, the results were observed to be inconsistent across the subjects and revealed an order effect. Similar results were reported by Hartmann, Rakerd, and Packard (1985). 


\section{Timbre}

Numerous studies have found that timbre influences efficiency and accuracy of pitch perception (Houtsma, 1997; Schubert, Wolfe, Tarnopolsky, 2004; Watts, Moore, \& McCaghren, 2005). Timbre, sometimes referred to as sound quality or sound color, is defined as the way in which sounds differ once they are equated for pitch, loudness, and duration. Seebeck (as cited in Boring, 1942) reported that fundamental frequency is not the only determinant of pitch but the upper harmonics also contribute to subjective pitch sensation. Beal (1985) suggested that it is difficult to filter out variations in timbre when judging pitch and also variations in pitch when judging timbre, especially for nonmusicians. Moore and Glasberg (1990) and Moore, Glasberg and Proctor (1992) measured thresholds for frequency discrimination using pairs of periodic sounds with the same or different spectral compositions. Higher thresholds were found when two sounds to be compared had different spectral compositions. This finding was interpreted as implying that pitch is not completely dissociable from timbre in the perception of sound. Watts et.al (2005) investigated the ability of accurate and inaccurate singers to categorize sounds that varied along pitch and timbre dimensions. They found differences between both groups' abilities to perceive disparities or similarities in stimulus pitch when there were timbre differences between the paired stimuli.

Platt and Racine (1985) investigated the ability of musicians to tune their instruments, i.e., to set the pitch of a sound, to match that of another reference sound. They concluded that this ability deteriorated when the sounds involved possessed different timbres. Crowder (1989), in his experiments on auditory imagery, used a task in which the subjects had to imagine tones played by different instruments. Initially, subjects were presented with a sine wave and asked to imagine this tone played by guitar, flute and trumpet. Then, they were presented with a second tone played by one of these instruments. The subjects' task was to judge whether the imagined tone and actual tones matched in pitch, as quickly as possible. He found that the judgments were faster and more accurate when the tones were played on the same instrument as the imagined tone (i.e., had the same timbre) than when played on different instruments from the imagined tone. He suggested that timbre variation interfered with the memory for pitch.

While there are many studies that have shown that differences in timbre can influence pitch perception, these studies have not employed experimental designs that allow us to determine, based on current theories of pitch perception, why timbre influences pitch perception. However, one study does examine the role of spectral centroid, or energy concentration, in pitch perception. Worthy (2000) investigated the effects of tone quality conditions on pitch perception and performance in 64 high school and college students. Results showed that subjects perceived tones with bright timbre (more energy at higher harmonics) as being sharper and tones with dark timbres (less energy at higher harmonics) as being flatter in pitch.

To further investigate the idea that spectral energy concentration affects pitch perception, Singh and Hirsh (1992) generated six types of stimuli with slightly different spectral energy concentrations. Each stimulus consisted of 4 harmonics, the first of 
harmonics 1-4, the second of harmonics 2-5, the third of harmonics 3-6, the fourth of harmonics 4-7, the fifth of harmonics 5-8, and the sixth of harmonics 6-9, thereby creating 6 complex stimuli with increasing spectral centroid. They also varied pitch. Generally, listeners perceived changes in spectral locus as changes in timbre and changes in $\mathrm{F}_{0}$ as changes in pitch. However, when the $\mathrm{F}_{0}$ change was small, less than $2 \%$, spectral centroid change was the primary predictor of pitch change. They interpreted this to mean that when $\mathrm{f}_{0}$ changes by less than $2 \%$, pitch and timbre are integrally perceived. When $\mathrm{f}_{0}$ changes by greater than $2 \%$, pitch and timbre are separable.

Russo and Thompson (2005) investigated the role of timbre on the perception of interval size. They created complex stimuli with a concentration of energy in the lower harmonics (dull) and stimuli with a concentration of energy in the higher harmonics (bright). They produced all possible pairs of stimuli at several pitch intervals and found that when the direction of the interval change was congruent to the pitch change (i.e., when the pitch went up, the timbre changed from dull to bright or when the pitch went down, the timbre changed from bright to dull) listeners heard larger pitch intervals than when the direction of the interval change was incongruent (when the pitch went up, the timbre changed from bright to dull or when the pitch went down the timbre changed from dull to bright). These results show that the concentration of spectral energy can interact with pitch interval perception in a manner consistent with the location of the spectral energy.

\section{Pitch Perception versus Production}

Vocal communication involves a complex interplay of perception and production systems that allow auditory-motor interaction to achieve sound targets. Among the brain regions intended for simple singing, the anterior cingulate cortex (ACC), superior temporal gyrus (STG), and insula may be candidates for vocal integration (Perry et.al, 1999). Research on the relationship between pitch perception and production has yielded contradictory results. Pitch discrimination and vocal pitch control have been proposed as independent abilities between which a relationship develops with training or maturation (Geringer, 1983; Goetze, Cooper, \& Brown, 1990; Yarbrough et.al, 1991).

In an attempt to determine the effects of maturation, Geringer (1983) investigated the interrelationship between pitch discrimination and vocal pitch matching ability among

preschool and fourth-grade students. He found that fourth-graders performed better on the pitch-matching task, but not on the pitch discrimination task. Also, there was no significant relationship between pitch discrimination scores and pitch-matching performance for either group, suggesting that while pitch discrimination develops early in life, pitch motor control develops at a slower rate.

Yarbrough and colleagues have investigated the possible effects of training on pitch perception and production. Two of their recent studies have compared the tuning accuracy of wind players using pitch perception and pitch production tasks (Yarbrough, Karrick, \& Morrison, 1995; Yarbrough, Morrison, \& Karrick, 1997). Subjects with 1-3 
years of training participated in the earlier study and subjects with 5, 6, and 7 years of training participated in the latter study. Students were asked to tune a target pitch using either their own instrument (production task) or a tuning knob on a variable-pitch keyboard (perception task). In both studies, years of experience significantly affected the tuning accuracy on both production and perception tasks. However, subjects did not show significant improvement in either of the tasks after 5 years of training. Also, there was no significant relationship between a subject's performance on the perception task and their performance on the production task.

However, other studies have suggested a relationship between production and perception. Pederson and Pederson (1970) used single pitches, melodic intervals, and three pitch sequences to examine the relationship between pitch discrimination and pitch matching skills among sixth grade students. They presented a series of standard and comparison stimuli and asked the subjects to judge if they were same or different. The study also included a vocal production task in which the subjects had to vocally produce a pitch that was played on a musical instrument. A moderate correlation was found to exist between pitch discrimination skills and vocal pitch matching ability.

Demorest (2001) further explored the relationship between perception and production by comparing junior high school boys' performance on a two-pitch matching tasks involving singing and tuning a knob. Results indicated a significant difference in perceptual task performance between singers who had been classified as certain in their pitch control and singers who had been classified as uncertain in their pitch control. In agreement with previous studies, Amir et al. (2003) also found that trained musicians demonstrated greater accuracy in pitch discrimination tasks and more accurate pitch matching ability than non-musicians.

\section{Vocal Pitch Matching}

The ability to match pitch is fundamental to the production of vocal music. In order to accurately match pitch, co-ordination of both sensory and motor systems are required. In a sequential pitch matching task, subjects must listen to the pitch and then store it in memory to reproduce it accurately. Then they must attempt to produce the pitch before either external or internal feedback can be utilized. It is at this point when motor programs are activated and an initial pitch is produced. Shortly after this initial pitch is produced, feedback, both auditory and kinesthetic, is available for more precise control of the pitch. Problems with pitch matching may arise due to problems in any of these areas. Other factors known to influence pitch matching ability are age, gender, and model characteristics. Factors known to influence pitch perception were discussed in a previous section. This section will focus on factors that may influence pitch matching from the memory stage to final production. 


\section{Memory}

In a sequential pitch matching task, listeners must store the target pitch in working memory. There are several competing theories of working memory. One model, proposed by Baddeley and Hitch (1974), assumes memory is a dynamic process involving two short-term storage systems, the phonological loop and the visuospatial sketchpad, and a higher level central executive control system that integrates information from both storage systems. The phonological loop is described as involving two subcomponents: a phonological store and an articulatory rehearsal component. The phonological store is hypothesized as acting somewhat like a "mind's ear" whereas the articulatory rehearsal is hypothesized as a mental rehearsal of the stimulus acting more as a "mind's voice" (Smith \& Kosslyn, 2008). Such a system would function equally well for short-term storage and processing of both phonological and musical information, so the name "phonological loop" might not apply literally; however, it is not clear whether similar parts of the brain would be activated in these two types of tasks.

Research in both humans and monkeys has supported the idea that working memory involves activation of the prefrontal cortex. While research in monkeys has suggested activation of the dorsal prefrontal cortex for spatial working memory and activation of the ventral prefrontal cortex for verbal and auditory tasks, studies in humans have suggested activation of the dorsal prefrontal cortex when working memory tasks require manipulation and activation of the ventral prefrontal cortex when working memory tasks require only maintenance.

Damage to the right frontal lobe has been shown to significantly affect pitch memory in humans and in animals (Gross \& Weiskrantz, 1962; Iversen \& Mishkin, 1973; Zatorre \& Samson, 1991). Using cerebral blood flow in passive listening and active task involving pitch memory, Zatorre and colleagues found increased activation of the right front lobe during task requiring use of working memory (Zatorre, et al, 1992; Zatorre, Evans, \& Meyer, 1994).

\section{Pre-phonatory Tuning}

In pitch matching tasks, an auditory stimulus is presented to participants and they are asked to vocally reproduce the fundamental frequency $\left(\mathrm{F}_{0}\right)$ of the tone as accurately as possible. This occurs during the few milliseconds at the beginning of vocalization. Leading up to this point in time, motor planning and programming has occurred that will govern the activation and position of laryngeal muscles and structures. The positioning of laryngeal structures prior to initiation of vocalization has been referred to in literature as pre-phonatory set or pre-phonatory tuning. The accuracy of pre-pre-phonatory tuning is measured in pitch matching paradigms by calculating the period of first measurable waveform of vocalization, converting this value to a corresponding frequency and comparing it against a target frequency. It has been found that singers are more accurate at pre-phonatory tuning than are non-singers (Leonard \& Ringel, 1979; Murry, 1990). 


\section{Auditory Feedback}

After the first few milliseconds of the pre-phonatory set phase of phonation, auditory feedback is available for use in modulating pitch control. It has been shown that it takes a minimum of 100 milliseconds of auditory feedback before a modulation occurs at the production level, rendering auditory feedback useless as a control mechanism in rapid speech (Fairbanks, 1955). However, because the durations of tones in singing can be much longer than 100 milliseconds, auditory feedback is likely an important control system for modulating pitch in singing. It has been found that when auditory feedback is masked, vocal $\mathrm{F}_{0}$ control becomes less accurate, reflecting the important interaction between ongoing auditory perception and vocal $\mathrm{F}_{0}$ accuracy (Elman, 1981; Hain et.al, 2000; Jones \& Munhall, 2000; Leydon, Bauer, \& Larson, 2003; Watts, Moore \& McCaghren, 2005).

\section{Age}

Most of the research on pitch-matching has been done with children in elementary school settings. Results from these studies have indicated that age is an important variable affecting pitch accuracy. Research studies show that pitch matching accuracy is enhanced with increasing age in the early elementary grades (Green, 1990; Klemish, 1974; Petzold, 1966).

\section{Gender}

Research on the effect of gender on pitch matching accuracy yielded inconsistent results (Yarbrough, Bowers \& Benson, 1992). Prominent among this research are findings showing that girls sing more on pitch than boys (Goetze, 1986; Green, 1993; Stauffer, 1985; Wilson, 1971). Remedial and instructional approaches to the improvement of vocal pitch matching accuracy have included those which use operant conditioning techniques such as reinforcement and successive approximation. Results of this research indicated that use of a vertical keyboard as reinforcement for correct pitch matching was helpful in pitch matching skills (Jones, 1979). However, the gain appears to be small and age appears to be a factor which may have a stronger influence on pitch matching ability than training.

\section{Model}

Even though pitch matching accuracy is considered a part of physical development and requires maturity, these skills are also influenced by the environment. Modeling is one of the important environmental variables that can affect pitch matching accuracy, although the research results are too varied to generalize the extent of the influence. Generally, there are two hypothesized mechanisms whereby the model might affect pitch matching. The first mechanism consists of low level processing of acoustic 
differences between the models. The second mechanism consists of higher level processing involving sensori-motor integration that is hypothesized to involve a possible mirror system in humans (Lévêque, Giovanni, \& Schön, 2011). Low level processes suggest that it is the difference in timbre between the model and the desired production that influence pitch matching, whereas, higher level processes, possibly involving mirror systems, might suggest that human pitch matching is optimized when the model is human.

Vocal compared to non-vocal stimuli. Some studies compare a vocal stimulus to a non-vocal stimulus. Weiner et.al (1996) demonstrated that the pitch matching abilities of graduate speech pathology students were more accurate when matching a human vocal stimulus compared to a pitch pipe. Lévêque, Giovanni, \& Schön (2011) tested whether auditory stimuli could be more accurately reproduced when the timbre is human than when the timbre is synthetic. Eighteen participants judged as poor singers and 14 controls were presented with vocal and synthetic singing models and had to reproduce them. Results showed that poor singers were significantly helped by human vocal model. The authors suggested that the effect of a human model on production might be linked to preactivation of motor representations during voice perception which may, in turn, facilitate the imitative vocal gesture. However, the effect of timbre difference cannot be excluded as a factor in either of these studies.

Vocal compared to a second vocal of differing timbre. Some studies compare vocal stimuli of different timbres. Green (1990) reported that the type of vocal modeling had an effect on pitch matching accuracy. He found that there were more correct responses to a child model followed by a female model and a male model, suggesting that timbre differences between the subject and the model influence pitch matching accuracy. However, he also found that the accuracy of pitch matching gradually increased with age in the case of a female model. He suggested that as listeners mature, they rely less on timbre in pitch matching.

Mixed models. However, most studies examining the effect of the model on pitch matching accuracy include human stimuli of differing timbres and instruments or synthetic stimuli. Depending on the design of the study, it is sometimes difficult to determine the cause of any resulting pitch matching errors, be it low level spectral processing or higher level sensori-motor integration.

One such study, Watts \& Hall (2008), investigated how the vocal fundamental frequency control of female undergraduate voice majors was influenced by the timbre of target auditory stimuli. They reported that participants were more accurate at matching the pitch of female target tones, followed by a male tone, a violin, and a clarinet. However, no direction correlation between spectral centroid difference and pitch matching ability was observed, although the two tones with the most different spectral centroid values, the violin and the clarinet, were also the two tones that were matched significantly less accurately than the female tones. From this study, it is not possible to determine whether timbre differences are responsible for inaccurate pitch matching or whether preference for a human model is responsible. 
Another study that employed vocal and non-vocal stimuli was conducted by Clegg (1966). She showed that the female voice was easier than a child's to match, followed by piano, auto harp and pitch pipe. The male voice, recorder, flutophone and song bells were more difficult for subjects to match.

A third study mixing vocal and non-vocal models was conducted by Hermanson (1972). He employed a slightly different methodology from others presented here. In his study, kindergarten and third grade children attempted to pitch match to a simultaneously-produced stimulus. He showed significant differences among responses to an oscillator, women's voice, piano and an 8-year old girl's voice. When singing simultaneously with different prerecorded pitch timbres, the subjects produced the most accurate pitches when singing with women's voice and the least accurate when singing with piano. He also reported that subjects were not significantly better at matching pitch with another child's voice than they were with stimuli with other timbres. Hermanson's findings do not support the idea that there is a preference for a human model, a suggestion made by the proponents of higher-level processing, and also do not support the idea that a stimulus with a timbre closer to the subject's timbre results in increased pitch matching, since the timbre of an 8 year old girl is likely to most closely match the timbre of kindergarten and third grade children. However, it may be that during simultaneous pitch matching, other factors become more important and so these findings may not be generalizable to sequential pitch matching tasks.

Vibrato. Stimulus models with vibrato have been shown to influence pitch matching. Yarbrough, Bowers \& Benson (1992) studied the effect of vibrato on the pitch matching accuracy in singers who were classified as more accurate (certain singers) and singers who were classified as less accurate (uncertain singers). Children from kindergarten through grade 6 were included in the study. Each subject had to match the pitch of target tones of three different models: child, female with vibrato and female without vibrato. In certain singers, there was no significant difference in pitch matching ability based on whether the model was a child model, a female singing with vibrato, or a female singing without vibrato. However, in uncertain singers, there were significant differences in pitch matching ability between these three models. Post-hoc analyses revealed that the non-vibrato model yielded a significantly greater number of correct responses than did the vibrato model for the uncertain singers. This suggests that while presence of vibrato may not be a factor in the pitch discrimination of singers whose intonation is more accurate, it may be a factor in the pitch matching of singers who have some difficulties with precise control of intonation.

Sundberg (1972) tested the effect of vibrato on pitch perception accuracy. In his experiment using pure tones, a stimulus tone and a response tone, both having vibrato modulation at the rate of 6.5 cycles per second and an extent of $1.7 \%$, were presented to the listeners. The listener's task was to adjust the response tone to match the pitch of the stimulus tone. Results of the experiment showed that the accuracy of pitch perception was not affected by the presence of vibrato. They also ran the experiment on one of the subjects using a vibrato extent of $\pm 3 \%$ as these higher vibrato rates occur commonly in singing. Even at a higher vibrato rate of $3 \%$, they did not find any significant alteration in 
pitch perception accuracy. Sundberg found that the perceived pitch was related to averaged fundamental frequency. However, this study employed sinusoidal stimuli only and may not generalize to complex sounds.

\section{Spectral Composition and Voice Classification}

Traditionally, singing voices have been divided into categories based on the classifications of bass, baritone, tenor, contralto, mezzo-soprano, and soprano. While these categories are typically defined according to pitch range, they are also defined by specific vocal timbres. Thus, a soprano and mezzo-soprano singing the same pitch will sound different based on timbre differences. Researchers have suggested that vocal tract length is one of the primary physiological predictors of voice category (Dmitriev \& Kiselev, 1979; Titze, 1994). Since formant frequency is dependent on vocal tract length, differences in vocal tract length will result in differences in timbre. Numerous researchers have demonstrated that formant frequency differences exist between the vocal categories (Berndtsson \& Sundberg, 1995; Cleveland, 1977; Dmitriev \& Kiselev, 1979; Erickson, 2004; Sundberg, 1973; Sundberg, 1994), with formant frequency values increasing in the order of bass, baritone, tenor, contralto, mezzo-soprano, and soprano.

\section{Purpose of the Study}

Based on numerous research studies, there is ample evidence that timbre perception has an influence on pitch matching ability. Singh and Hirsh (1992) demonstrated that when the $\mathrm{F}_{0}$ difference between 2 stimuli is small, less than $2 \%$, spectral centroid change is the primary predictor of perceived pitch change. Given this, it might be reasonable to assume that trained singers, whose internal pitch models likely will not differ from the target by more than $2 \%$ will be able to more accurately match pitch if the vocal model presented has a timbre closer to their voice type. It is a commonly held belief that mezzo-sopranos are sometimes judged as being "flat" compared to sopranos. Based on the work of Worthy (2000), it might be expected that mezzo-sopranos, with lower formant frequencies than sopranos, would sing sharp when imitating a soprano's voice and sopranos would sing flat when imitating a mezzosoprano's voice.

Pitch is also likely to be a factor in a pitch matching task. Because the timbre of a singing voice is an interaction between the source slope and the formant frequencies, spectral centroid differences between target stimuli and experimental stimuli will likely vary with pitch, since at higher pitches, harmonics often do not align with formants, thereby reducing the spectral information related to their location. In such cases, the spectral centroid difference between target and experimental production may be reduced compared to lower pitches.

The effect of vibrato on a pitch matching task in adult trained singers is less certain. Using pure tones only, Sundberg (1972) found no effect of vibrato on pitch 
matching ability; however, in young singers with poor pitch matching skills, Yarbrough and colleagues (1992) found that the ability to pitch match was reduced when the target stimulus was produced with vibrato.

If accuracy of pitch matching is affected by a small change in spectral composition or change in timbre, we can say that singers are extracting pitch from a representation of spectral profile of the model, supporting spectral theories of pitch perception. If the accuracy of pitch matching does not change with a small change in spectral composition, then it might be the temporal analysis that hold true in terms of pitch perception.

The present study seeks to answer the following questions:

1) Do classically trained female singers more accurately match pitch when the target stimulus is more similar to their own timbre?

2) Does the ability to match pitch vary with increasing pitch?

3) Does pitch matching ability differ depending on whether the target stimulus is produced with or without vibrato?

4) Are mezzo-sopranos less accurate than sopranos at (1) pre-phonatory set or (2) the mid-point of the vowel?

A hypothetical representation of the proposed phenomenon is presented graphically in Figure 1 where singers are more in tune when the target formant pattern is close to their own vocal timbre. In Figure 1, X-axis represents formant pattern, a measure of target timbre. Y-axis represents mean difference in cents reflecting pitch matching accuracy. If the hypotheses was true, we expect that mezzo-sopranos are most in tune at pattern $\mathrm{A}$ and are sharp at pattern $\mathrm{E}$ and sopranos are flat at pattern $\mathrm{A}$ and most in tune at pattern $\mathrm{E}$. 


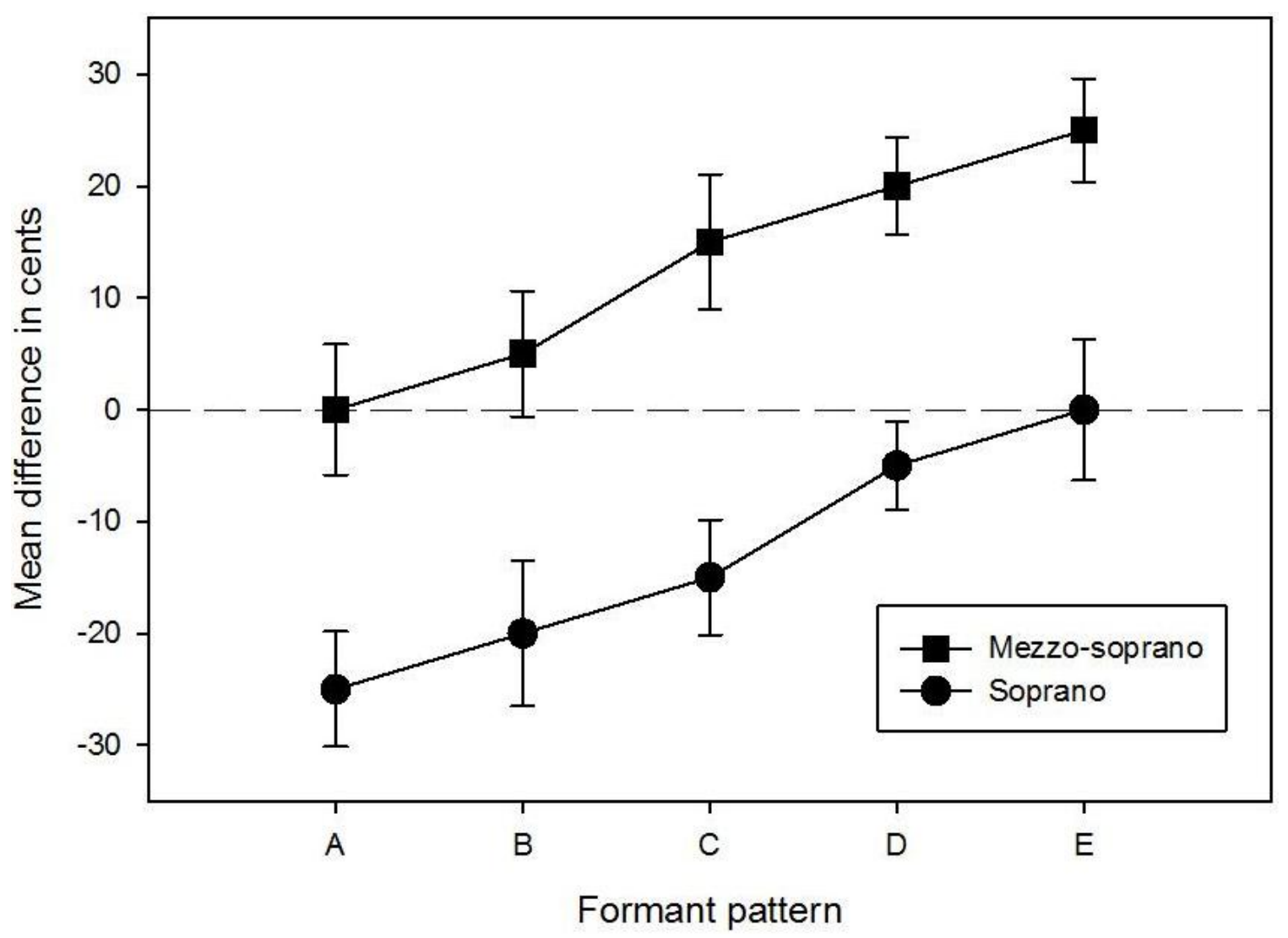

Figure 1. Hypothetical representation showing enhanced pitch matching ability from formant pattern A to formant pattern $E$. 


\section{CHAPTER 3. METHOD}

\section{Stimuli}

The stimuli were previously generated synthetically using an analogue digital synthesizer. The synthesis model was built using Aladdin Interactive DSP work bench (Hi-tech development, Stockholm, Sweden). The Aladdin synthesizer produces synthetic singing voice stimuli that are extremely realistic and are not easily identified as synthetic. For the pitches C4, B4, and F5, source signals were synthesized with a slope of -12 $\mathrm{dB} /$ octave using vibrato and no-vibrato, for a total of 6 source signals. For the vibrato source signals, a frequency vibrato rate of $5.6 \mathrm{~Hz}$ and a frequency vibrato extent of 50 cents were used. At each pitch, source signals were filtered using 5 formant patterns (pattern A through E) for the vowel/a/. Pattern A is representative of that typically seen in a mezzo-soprano. Pattern $\mathrm{E}$ is representative of a formant pattern typically seen in a soprano. Patterns B-D were interpolated using a linear frequency scale to fall at equal intervals between patterns A and E. Formant patterns A-E are displayed in Table 1. This resulted in 5 stimuli for each of the 3 pitches in both vibrato and no-vibrato conditions, for a total of 30 stimuli. Using Adobe Audition (Salt Lake City, Utah), each stimulus was edited to one second in duration, smoothed using spline curves applied to the onset and offset, and normalized in RMS amplitude.

\section{Participants}

Twelve sopranos and 11 mezzo-soprano participated in the study $(\mathrm{n}=23)$. Listeners were recruited from the University Of Tennessee School Of Music and the Knoxville Opera Company. All of them had at least 3 years of formal training in singing. Each singer was in the age range of 20-55 years. They did not have any history of vocal problems and passed a hearing screening.

Table 1. Formant patterns A and B.

\begin{tabular}{ccccc}
\hline Pattern & F1 & F2 & F3 & F4 \\
\hline A & 625 & 1074 & 3027 & 3600 \\
B & 680 & 1141 & 3098 & 3674 \\
C & 741 & 1212 & 3170 & 3749 \\
D & 806 & 1287 & 3244 & 3827 \\
E & 878 & 1367 & 3320 & 3906 \\
\hline
\end{tabular}




\section{Procedure}

Participants provided informed consent using a procedure that has been approved by institutional review board at University of Tennessee Health Science Center. For each subject, audiometric screening was performed at $250 \mathrm{~Hz}, 1 \mathrm{kHz}, 2 \mathrm{kHz}, 4 \mathrm{KHz}$ and $8 \mathrm{kHz}$ to ensure normal bilateral hearing acuity. Each singer was presented with all 30 stimuli in a randomized order with each stimulus appearing twice, for a total of 60 trials. Each stimulus was presented in rhythmic pattern 4 times and the singer was expected to imitate pitch the fifth time. A noise of 1 second in duration was presented before each stimulus set in order to mask the pitch of the previous stimulus set.

Differences in mean F0 between the target and experimental stimuli was calculated in an attempt to measure the accuracy of pitch matching. This difference in mean F0 was measured at 2 locales: (1) pre-phonatory set, (2) mid-point of the vowel. Pitch matching abilities at the pre-phonatory set was measured by converting the period of the first measurable waveform into a corresponding frequency. This value was then subtracted from the frequency of target tone so that a difference score was obtained for each response. The difference score was converted into cents. This difference score reflected pitch matching accuracy. Greater the difference in F0 between target stimuli and experimental productions, poorer the pitch matching accuracy. Similarly, mean $\mathrm{F}_{0}$ was measured at the mid-point of the vowel of experimental stimuli and was subtracted from the mean $\mathrm{F}_{0}$ at the same point of target stimuli. This difference was also converted to cents. Mean centroid values were also calculated for both mezzo-sopranos and sopranos to show that there is significant voice quality difference between the groups. An overview of statistical analysis is presented in the Figure 2.

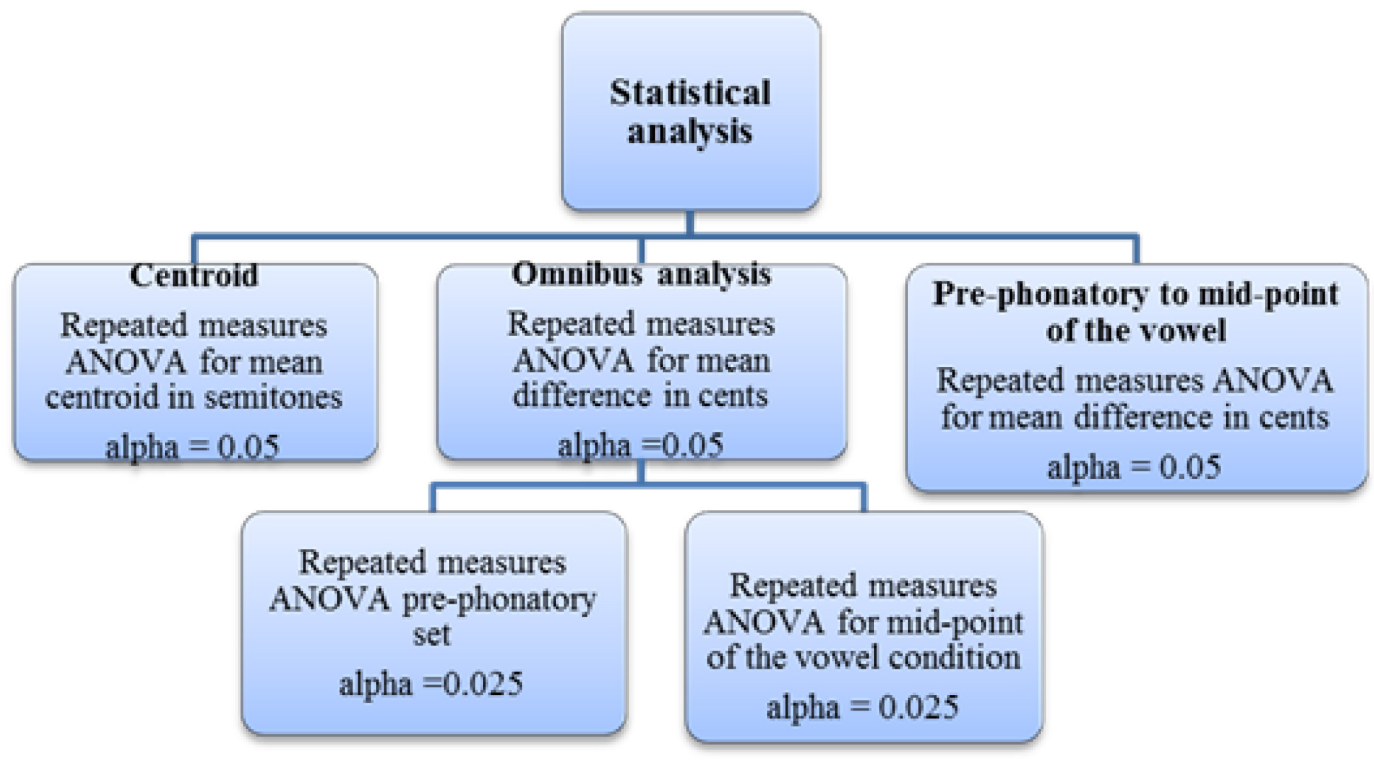

Figure 2. Overview of statistical analysis including Bonferroni corrections at each level of analysis. 


\section{CHAPTER 4. RESULTS}

\section{Centroid}

To test whether or not a significant voice quality difference existed between the mezzo-soprano and soprano participants, a $2 \times 2 \times 3 \times 5$ repeated measures ANOVA was conducted investigating the effects of the between-groups factor, voice category and the repeated measures factors, pitch, vibrato and formant pattern ( A, B, C, D and E), on mean centroid in semitones (with $440 \mathrm{~Hz}$ as reference). The results are presented in Table

2. There was a significant main effect of voice category on mean spectral centroid $\left(F_{(1,21)}\right.$ $=5.355, p=.031)$. Also, the effect of formant pattern was significant $\left(\mathrm{F}_{(4,84)}=2.475\right.$, $p=.050$ ). However, a significant interaction between formant pattern and vibrato was observed $\left(\mathrm{F}_{(4,84)}=3.196, p=0.017\right)$. Mean centroid values for mezzo-sopranos and sopranos as a function of formant pattern and vibrato are displayed in Figures 3-5 for pitches $\mathrm{C} 4$, B4 and $\mathrm{F} 5$, respectively.

In Figures 3-5, the $\mathrm{X}$-axis represents the stimulus formant pattern and $\mathrm{Y}$-axis represents the difference in mean centroid in semitones between the experimental productions and a standard reference of frequency $440 \mathrm{~Hz}$. A value of zero implies centroid of the experimental production is equal to the reference frequency $(440 \mathrm{~Hz})$. A positive value of ' $n$ ' implies that experimental productions are ' $n$ ' semitones higher than $440 \mathrm{~Hz}$. A negative value of ' $n$ ' implies that experimental productions are ' $n$ ' semitones lower than $440 \mathrm{~Hz}$. All three figures indicate that mezzo-sopranos have significantly lower centroids than sopranos.

Further, separate post-hoc ANOVA procedures were conducted to test the effect of formant pattern on mean centroid values for vibrato and no-vibrato conditions. Results of the post-hoc analyses revealed a significant main effect of formant pattern in vibrato condition $\left(\mathrm{F}_{(4,84)}=5.036, p=.001\right)$. The results of post-hoc ANOVAs are presented in Tables 3 and $\mathbf{4}$ for vibrato and no-vibrato conditions, respectively. Mean centroid in semitones as a function of formant pattern averaged across pitch is represented in Figures 6 and 7 for vibrato and no-vibrato condition, respectively. No significant effect of formant pattern on spectral centroid was seen in no-vibrato condition.

Based on the within-subjects contrasts, a significant linear relationship between mean centroid and target formant pattern was observed $\left(\mathrm{F}_{(1,21)}=8.694, p=.008\right)$. As the formant pattern increased from $\mathrm{A}$ to $\mathrm{E}$, the mean centroid values also increased in a linear manner.

\section{Omnibus Analysis}

The overall results of $2 \times 2 \times 2 \times 3 \times 5$ repeated measures ANOVA for difference in cents between the target stimuli and the experimental productions with voice category as a between-groups factor and the repeated measures of condition (pre-phonatory and mid- 
Table 2. Results of $2 \times 3 \times 5 \times 2$ ANOVA for mean centroid in semitones.

\begin{tabular}{lcccc}
\hline Effect & F & Hypotheses df & Error df & Sig. \\
\hline Category & 5.355 & 1 & 21 & $.031^{*}$ \\
Pitch & 1.898 & 2 & 42 & .162 \\
Pitch*category & 1.926 & 2 & 42 & .158 \\
Pattern & 2.475 & 4 & 84 & $.050^{*}$ \\
Pattern*category & .879 & 4 & 84 & .480 \\
Vibrato & 3.834 & 1 & 21 & .066 \\
Vibrato*category & 1.314 & 1 & 21 & .265 \\
Pitch*pattern & 1.360 & 8 & 168 & .218 \\
Pitch*pattern*category & .834 & 8 & 168 & .574 \\
Pitch*vibrato & .369 & 2 & 42 & .693 \\
Pitch*vibrato*category & 3.083 & 2 & 42 & .056 \\
Pattern*vibrato & 3.196 & 4 & 84 & $.017 *$ \\
Pattern*vibrato*category & 1.616 & 4 & 84 & .178 \\
Pitch*pattern*vibrato & 1.170 & 8 & 168 & .320 \\
Pitch*pattern*vibrato* & .556 & 8 & 168 & .813 \\
category & & & & \\
\hline
\end{tabular}

*= significance at alpha 0.05

$\mathrm{F}=$ between groups/within groups variance

$\mathrm{df}=$ degrees of freedom 


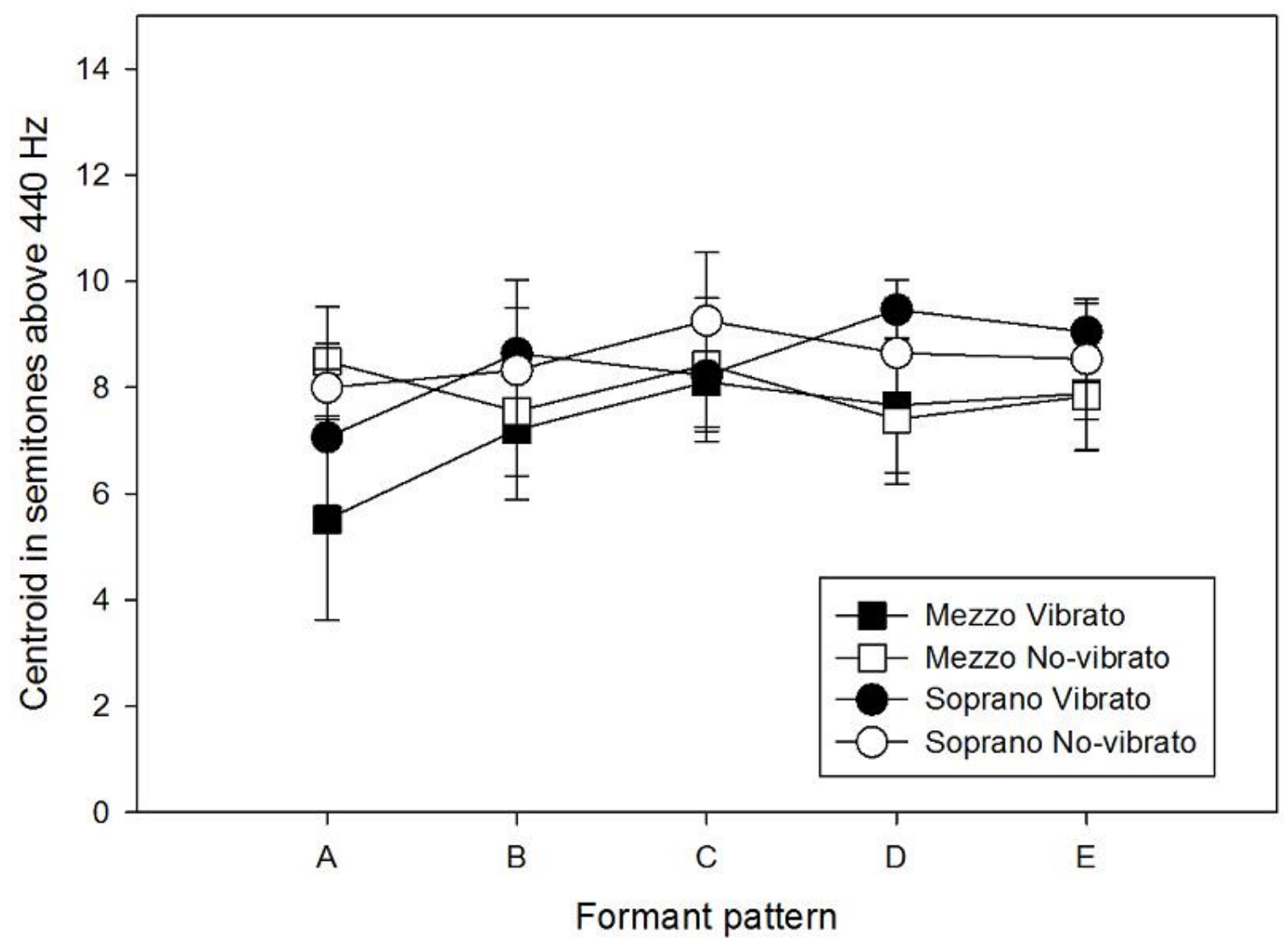

Figure 3. Mean centroid in semitones for mezzo-sopranos and sopranos as a function of formant pattern and vibrato at pitch $\mathrm{C} 4$. 


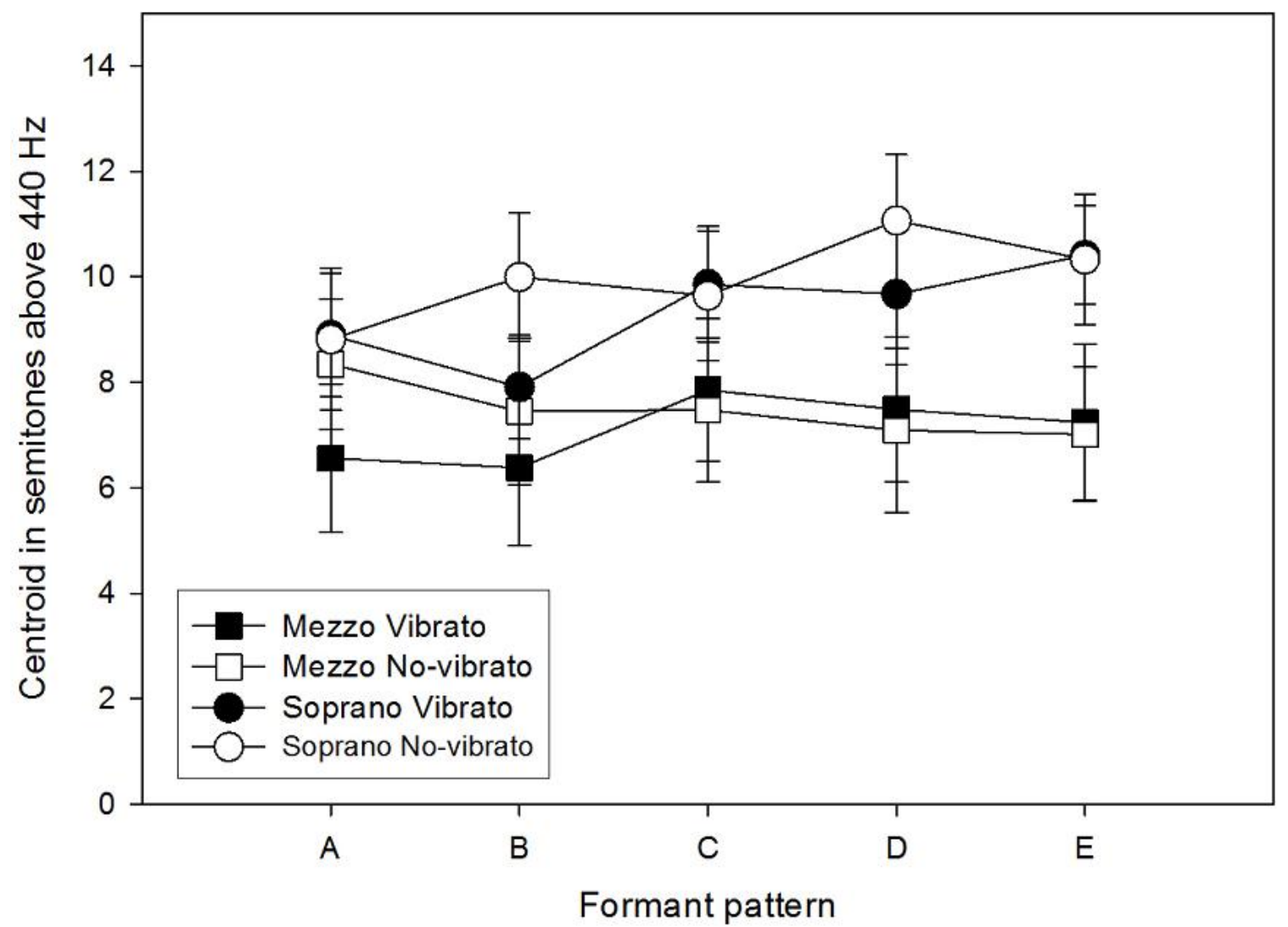

Figure 4. Mean centroid in semitones for mezzo-sopranos and sopranos as a function of formant pattern and vibrato at pitch $B 4$. 


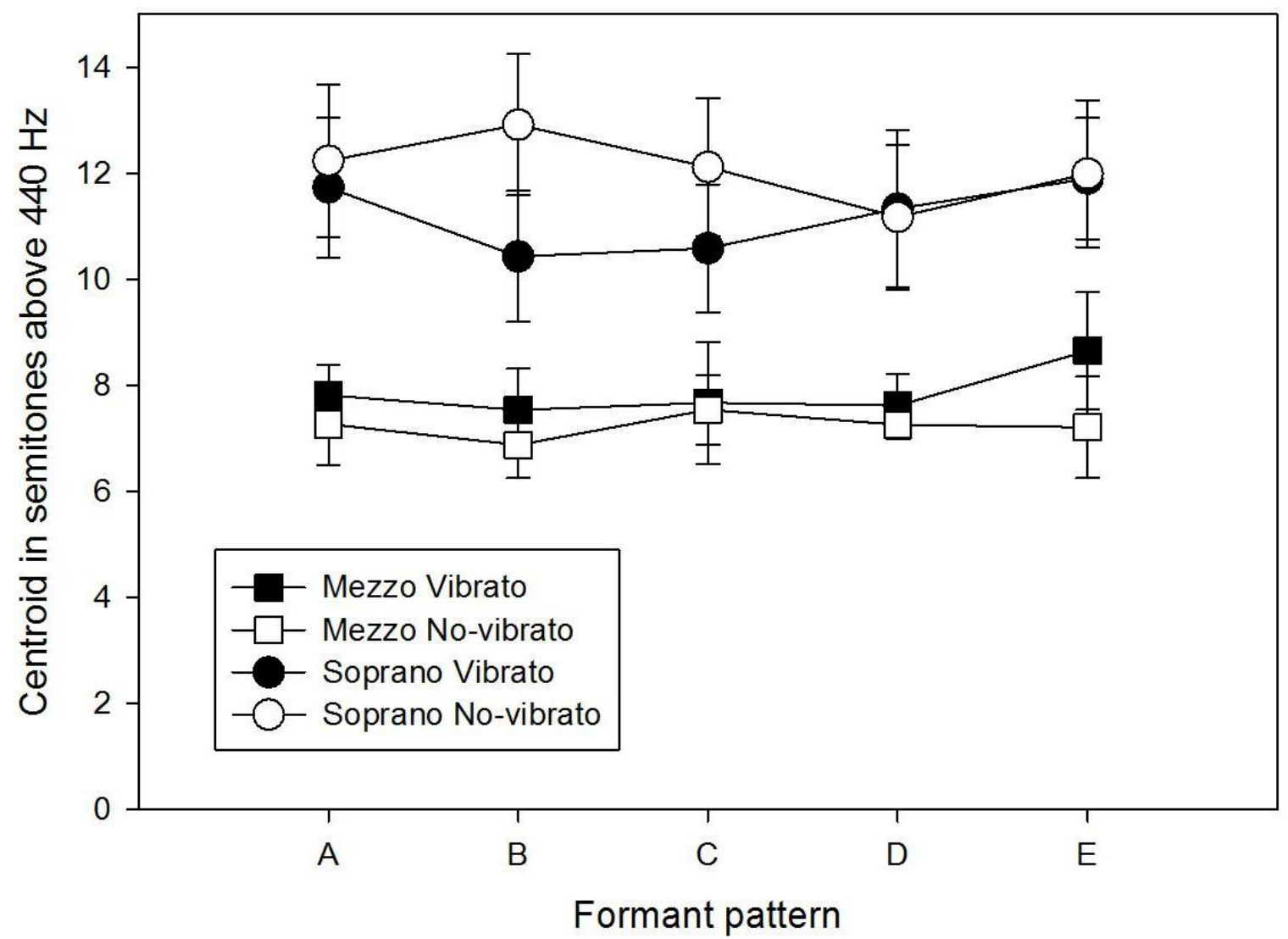

Figure 5. Mean centroid in semitones for mezzo-sopranos and sopranos as a function of formant pattern and vibrato at pitch F5. 
Table 3. Results of post-hoc ANOVA for mean centroid as a function of pitch and pattern in vibrato condition.

\begin{tabular}{lcccc}
\hline Effect & F & Hypotheses df & Error df & Sig. \\
\hline Category & 4.573 & 1 & 21 & .044 \\
Pitch & 2.144 & 2 & 42 & .130 \\
Pitch*category & .802 & 2 & 42 & .455 \\
Pattern & 5.036 & 4 & 84 & $.001 *$ \\
Pattern*category & .736 & 4 & 84 & .570 \\
Pitch*pattern & 1.800 & 8 & 168 & .080 \\
Pitch*pattern*category & .265 & 8 & 168 & .976 \\
\hline
\end{tabular}

*= significance at alpha 0.025

$\mathrm{F}=$ between groups/within groups variance

$\mathrm{df}=$ degrees of freedom

Table 4. Results of post-hoc ANOVA for mean centroid as a function of pitch and pattern in no-vibrato condition.

\begin{tabular}{lcccc}
\hline Effect & F & Hypotheses df & Error df & Sig. \\
\hline Category & 5.897 & 1 & 21 & $.024 *$ \\
Pitch & 1.455 & 2 & 42 & .245 \\
Pitch*category & 3.190 & 2 & 42 & .051 \\
Pattern & .941 & 4 & 84 & .904 \\
Pattern*category & 1.744 & 4 & 84 & .148 \\
Pitch*pattern & .570 & 8 & 168 & .802 \\
Pitch*pattern*category & 1.188 & 8 & 168 & .309 \\
\hline
\end{tabular}

*= significance at alpha 0.025

$\mathrm{F}=$ between groups/within groups variance

$\mathrm{df}=$ degrees of freedom 


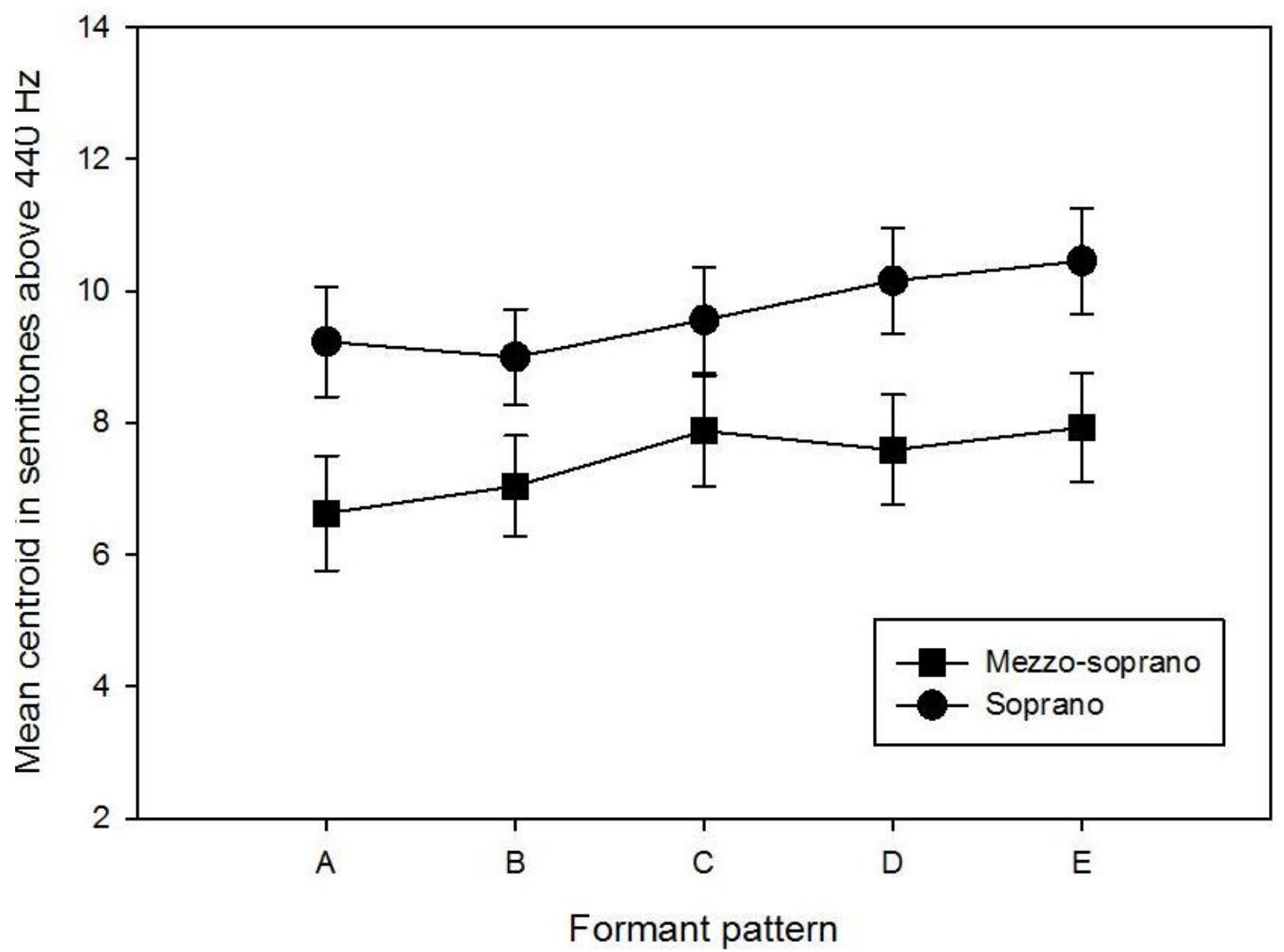

Figure 6. Mean centroid in semitones as a function of formant pattern in vibrato condition. 


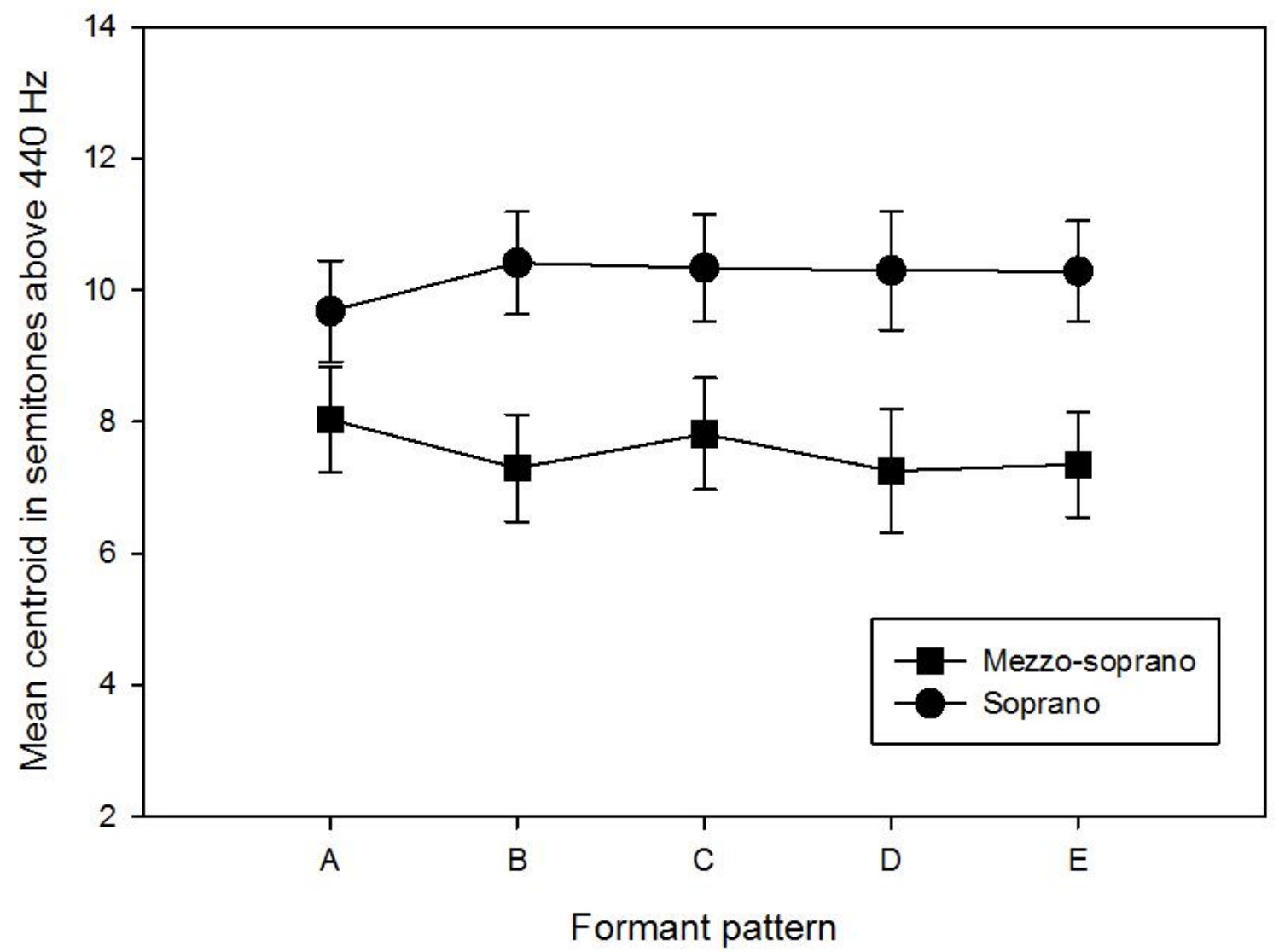

Figure 7. Mean centroid in semitones as a function of formant pattern in novibrato condition. 
point of the vowel), pitch, formant pattern, and vibrato is shown in Table 5. A significant difference between the pre-phonatory condition and mid-point of the vowel condition was found $\left(\mathrm{F}_{(1,21)}=27.929, p<.001\right)$. Also, a significant effect of pitch was seen $\left(\mathrm{F}_{(2,42)}\right.$ $=20.121, p<.001)$. However, there were numerous significant interactions involving both condition and pitch. The interaction between condition and pitch was significant $\left(\mathrm{F}_{(1,21)}\right.$ $=20.591, p<.001)$. The interaction between pitch and vibrato was also significant $\left(\mathrm{F}_{(2,42)}\right.$ $=5.570, p=.007)$. Finally, a significant interaction between condition, pitch and vibrato was observed $\left(\mathrm{F}_{(2,42)}=5.435, p=.008\right)$. No significant main effects of vibrato or pattern were seen.

Post-hoc ANOVAs were performed to investigate the effect of condition on mean difference in cents at each of the 3 pitches, C4, B4 and F5. The results of these analyses are presented in Tables 6-8 for C4, B4 and F5, respectively. The results indicated that there is significant main effect of condition on mean difference in cents at all the pitches.

\section{Pre-phonatory Condition}

The results of $2 \times 2 \times 3 \times 5$ repeated measures ANOVA for mean difference in cents between the target stimuli and the experimental productions in the pre-phonatory condition are shown in Table 9. No significant difference between the sopranos and mezzo-sopranos was found. Also, no significant effect of pitch, vibrato or pattern was seen. Mean difference in cents between the target and the experimental productions in mezzo-sopranos and sopranos as a function of vibrato and formant pattern is displayed in the Figures 8-10 for pitches $\mathrm{C} 4$, B4 and F5, respectively.

In the Figures 8-10, the $\mathrm{X}$-axis represents the target formant pattern and the $\mathrm{Y}$ axis represents the mean difference in cents between the target stimuli and the experimental productions. On the $\mathrm{Y}$-axis, a value of zero indicates that on average the experimental production is equal to the frequency of target stimuli. A negative value implies that the mean experimental production was lower in pitch than the target stimuli. A positive value implies that the mean experimental production was higher in pitch than the target stimuli.

\section{Mid-point of the Vowel Condition}

The results of $2 \times 2 \times 3 \times 5$ repeated measures ANOVA for mean difference in cents between the target stimuli and the experimental production for the mid-point of the vowel condition are given in Table 10. No significant difference between mezzo-sopranos and sopranos was found. However, the effect of pitch was significant $\left(\mathrm{F}_{(2,42)}=20.360, \mathrm{P}\right.$ $<0.001)$. Also, a significant interaction was found between pitch and vibrato $\left(\mathrm{F}_{(2,42)}=\right.$ $5.507, \mathrm{P}<0.05)$. Mean difference in cents between the target and the experimental productions in mezzo-sopranos and sopranos as a function of vibrato and formant pattern is displayed in the Figures 11-13 for pitches C4, B4, and F5, respectively. 
Table 5. Results of $2 \times 2 \times 2 \times 3 \times 5$ repeated measures ANOVA for difference in cents between the target stimuli and the experimental productions.

\begin{tabular}{|c|c|c|c|c|}
\hline Effect & $\mathrm{F}$ & Hypotheses df & Error df & Sig. \\
\hline Category & .463 & 1 & 21 & .504 \\
\hline Condition & 27.929 & 1 & 21 & $<.001 *$ \\
\hline Condition* category & .535 & 1 & 21 & .472 \\
\hline Pitch & 20.121 & 2 & 42 & $<.001 *$ \\
\hline Pitch*category & .343 & 2 & 42 & .711 \\
\hline Pattern & .474 & 4 & 84 & .755 \\
\hline Pattern*category & .787 & 4 & 84 & .537 \\
\hline Vibrato & .449 & 1 & 21 & .510 \\
\hline Vibrato*category & .062 & 1 & 21 & .805 \\
\hline Condition*pitch & 20.591 & 2 & 42 & $<.001^{*}$ \\
\hline Condition*pitch*category & .336 & 2 & 42 & .717 \\
\hline Condition*pattern & .472 & 4 & 84 & .756 \\
\hline Condition*pattern*category & .807 & 4 & 84 & .524 \\
\hline Pitch*pattern & 1.782 & 8 & 168 & .084 \\
\hline Pitch*pattern*category & .308 & 8 & 168 & .962 \\
\hline Condition*Pitch*Pattern & 1.804 & 8 & 168 & .079 \\
\hline Condition*pitch*pattern* category & .339 & 8 & 168 & .950 \\
\hline Condition*vibrato & .456 & 1 & 21 & .507 \\
\hline Condition*vibrato* category & .040 & 1 & 21 & .844 \\
\hline Pitch*vibrato & 5.570 & 2 & 42 & $.007 *$ \\
\hline Pitch*vibrato*category & .265 & 2 & 42 & .768 \\
\hline Condition*pitch*vibrato & 5.435 & 2 & 42 & $.008 *$ \\
\hline Condition* pitch*vibrato* category & .209 & 2 & 42 & .812 \\
\hline Pattern*vibrato & .178 & 4 & 84 & .949 \\
\hline
\end{tabular}


Table 5. Continued.

\begin{tabular}{lcccc}
\hline Effect & F & Hypotheses df & Error df & Sig. \\
\hline Pattern*vibrato*category & .203 & 4 & 84 & .936 \\
Condition*pattern*vibrato & .156 & 4 & 84 & .960 \\
Condition*pattern*vibrato*category & .195 & 4 & 84 & .940 \\
Pitch*pattern*vibrato & 1.158 & 8 & 168 & .328 \\
$\begin{array}{l}\text { Pitch*Pattern*vibrato*category } \\
\text { Condition*pitch*pattern*vibrato }\end{array}$ & .851 & 8 & 168 & .560 \\
$\begin{array}{l}\text { Condition*pitch*pattern*vibrato* } \\
\text { category }\end{array}$ & .887 & 8 & 168 & .307 \\
\hline
\end{tabular}

*= significance at alpha 0.05

$\mathrm{F}=$ between-groups/within-groups variance

$\mathrm{df}=$ degrees of freedom 
Table 6. Results of post-hoc ANOVA for mean difference in cents between target stimuli and experimental productions at pitch $\mathrm{C} 4$.

\begin{tabular}{lcccc}
\hline Effect & F & Hypotheses df & Error df & Sig. \\
\hline Category & .094 & 1 & 21 & .763 \\
Condition & 63.859 & 1 & 21 & $<.001 *$ \\
Condition*category & .136 & 1 & 21 & .716 \\
Pattern & .747 & 4 & 84 & .563 \\
Pattern*category & .622 & 4 & 84 & .648 \\
Vibrato & 6.107 & 1 & 21 & .022 \\
Vibrato*category & .001 & 1 & 21 & .979 \\
Condition*pattern & .796 & 4 & 84 & .531 \\
Condition*pattern*category & .650 & 4 & 84 & .628 \\
Condition*vibrato & 6.028 & 1 & 21 & .023 \\
Condition*vibrato*category & .003 & 1 & 21 & .959 \\
Pattern*vibrato & 1.571 & 4 & 84 & .189 \\
Pattern*vibrato*category & .715 & 4 & 84 & .584 \\
Condition*pattern*vibrato & 1.684 & 4 & 84 & .161 \\
Condition*pattern*vibrato*category & .726 & 4 & 84 & .577 \\
\hline
\end{tabular}

*= significance at alpha 0.016 after Bonferroni correction

$\mathrm{F}=$ between-groups/within- groups variance

$\mathrm{df}=$ degrees of freedom 
Table 7. Results of post-hoc ANOVA for mean difference in cents between target stimuli and experimental productions at pitch B4.

\begin{tabular}{lcccc}
\hline Effect & F & Hypotheses df & Error df & Sig. \\
\hline Category & .816 & 1 & 21 & .377 \\
Condition & 12.166 & 1 & 21 & $.002 *$ \\
Condition*category & .911 & 1 & 21 & .351 \\
Pattern & .092 & 4 & 84 & .985 \\
Pattern*category & .442 & 4 & 84 & .778 \\
Vibrato & 1.826 & 1 & 21 & .191 \\
Vibrato*category & .078 & 1 & 21 & .783 \\
Condition*pattern & .063 & 4 & 84 & .992 \\
Condition*pattern*category & .492 & 4 & 84 & .741 \\
Condition*vibrato & 1.691 & 1 & 21 & .208 \\
Condition*vibrato*category & .091 & 1 & 21 & .766 \\
Pattern*vibrato & .441 & 4 & 84 & .778 \\
Pattern*vibrato*ategory & .698 & 4 & 84 & .595 \\
Condition*pattern*vibrato & .378 & 4 & 84 & .824 \\
Condition*pattern*vibrato*category & .669 & 4 & 84 & .615 \\
\hline
\end{tabular}

*= significance at alpha 0.016 after Bonferroni correction

$\mathrm{F}=$ between-groups/within- groups variance

$\mathrm{df}=$ degrees of freedom 
Table 8. Results of post-hoc ANOVA for mean difference in cents between target stimuli and experimental productions at pitch F5.

\begin{tabular}{|c|c|c|c|c|}
\hline Effect & $\mathrm{F}$ & Hypotheses df & Error df & Sig. \\
\hline Category & .307 & 1 & 21 & .585 \\
\hline Condition & 7.081 & 1 & 21 & $.015^{*}$ \\
\hline Condition*category & .332 & 1 & 21 & .571 \\
\hline Pattern & 2.848 & 4 & 84 & .029 \\
\hline Pattern*category & .394 & 4 & 84 & .812 \\
\hline Vibrato & .310 & 1 & 21 & .584 \\
\hline Vibrato* category & .729 & 1 & 21 & .403 \\
\hline Condition*pattern & 2.850 & 4 & 84 & .029 \\
\hline Condition*pattern*category & .403 & 4 & 84 & .806 \\
\hline Condition*vibrato & .384 & 1 & 21 & .542 \\
\hline Condition*vibrato*category & .535 & 1 & 21 & .472 \\
\hline Pattern*vibrato & .612 & 4 & 84 & .655 \\
\hline Pattern*vibrato*category & .546 & 4 & 84 & .702 \\
\hline Condition*pattern*vibrato & .621 & 4 & 84 & .649 \\
\hline Condition*pattern*vibrato* category & .643 & 4 & 84 & .633 \\
\hline
\end{tabular}


Table 9. Results of $2 \times 3 \times 5 \times 2$ ANOVA for mean difference in cents between the target and the experimental productions in pre-phonatory condition.

\begin{tabular}{|c|c|c|c|c|}
\hline Effect & $\mathrm{F}$ & Hypotheses df & Error df & Sig. \\
\hline Category & 2.397 & 1 & 21 & .137 \\
\hline Pitch & .068 & 2 & 42 & .935 \\
\hline Pitch* category & 1.161 & 2 & 42 & .323 \\
\hline Pattern & .516 & 4 & 84 & .724 \\
\hline Pattern*category & 1.164 & 4 & 84 & .333 \\
\hline Vibrato & .014 & 1 & 21 & .907 \\
\hline Vibrato*category & .537 & 1 & 21 & .472 \\
\hline Pitch*pattern & .858 & 8 & 168 & .554 \\
\hline Pitch*pattern* category & .903 & 8 & 168 & .516 \\
\hline Pitch*vibrato & .712 & 2 & 42 & .497 \\
\hline Pitch*vibrato*category & 1.376 & 2 & 42 & .264 \\
\hline Pattern*vibrato & .453 & 4 & 84 & .770 \\
\hline Pattern*vibrato*category & .560 & 4 & 84 & .692 \\
\hline Pitch*pattern*vibrato & 1.926 & 8 & 168 & .059 \\
\hline Pitch*pattern*vibrato* category & .979 & 8 & 168 & .454 \\
\hline
\end{tabular}




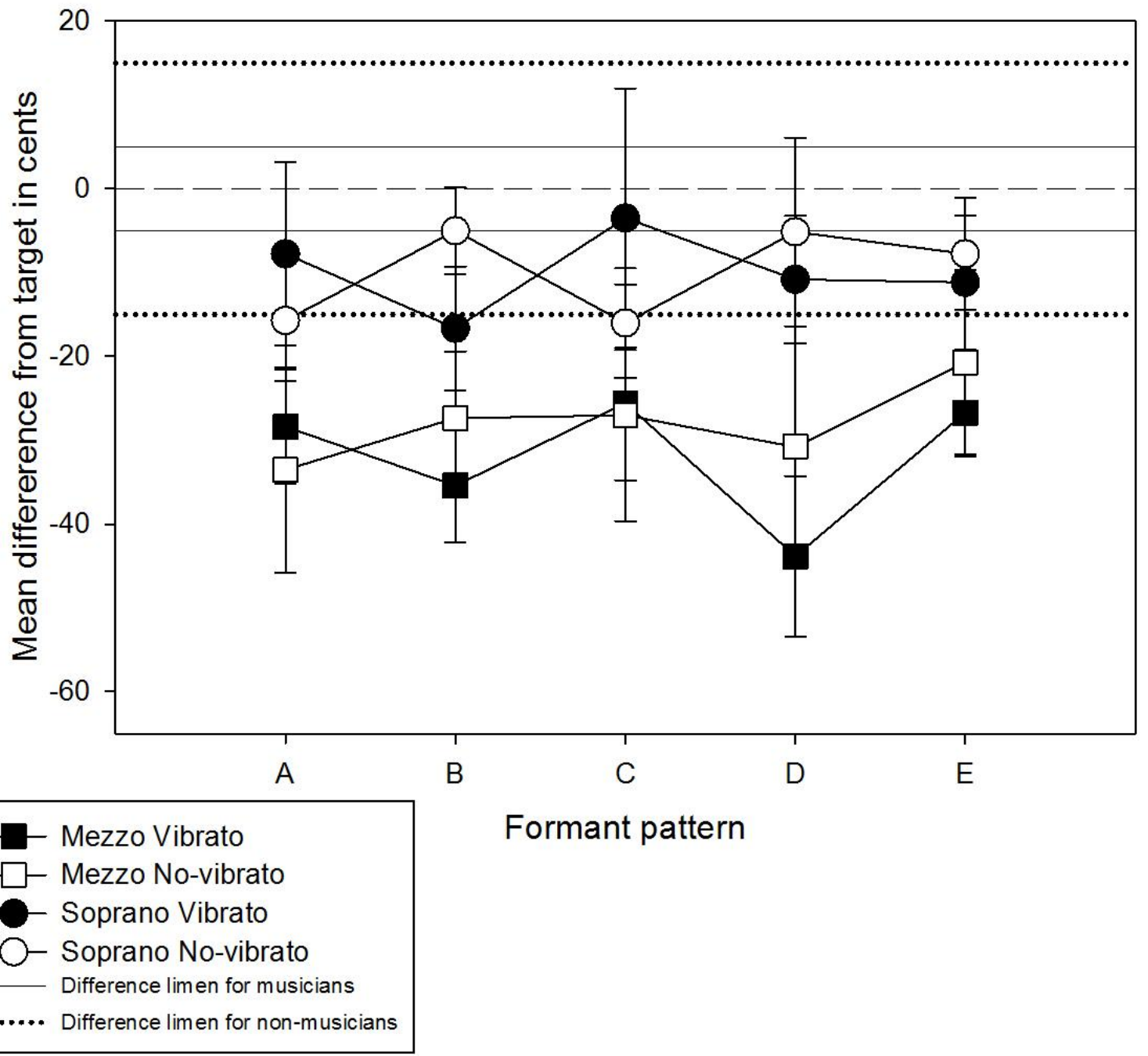

Figure 8. Mean difference in cents between the target and the experimental productions as a function of formant pattern and vibrato at pitch $\mathrm{C} 4$ in prephonatory condition. 


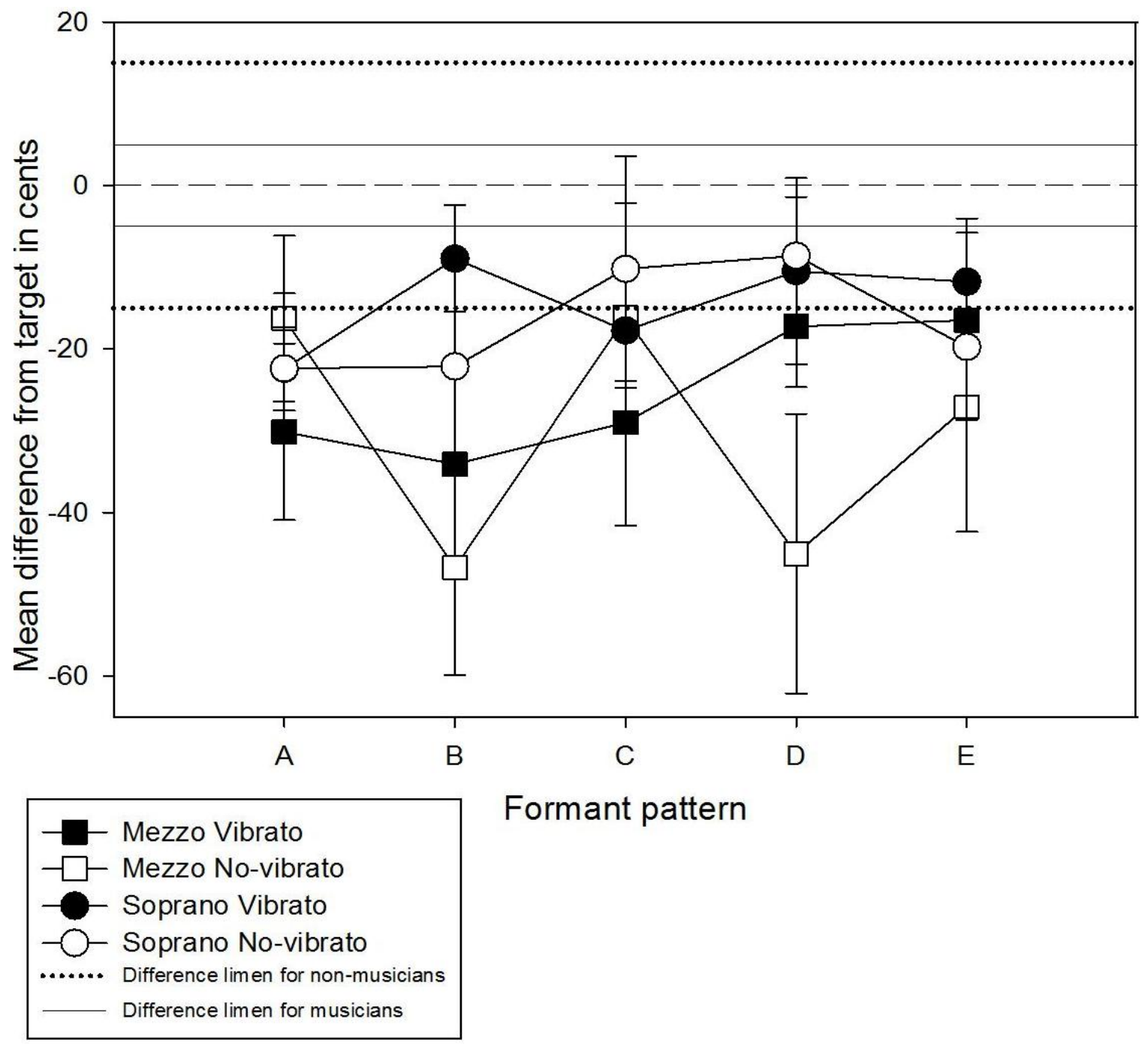

Figure 9. Mean difference in cents between the target and the experimental productions as a function of formant pattern and vibrato at pitch $B 4$ in prephonatory condition. 


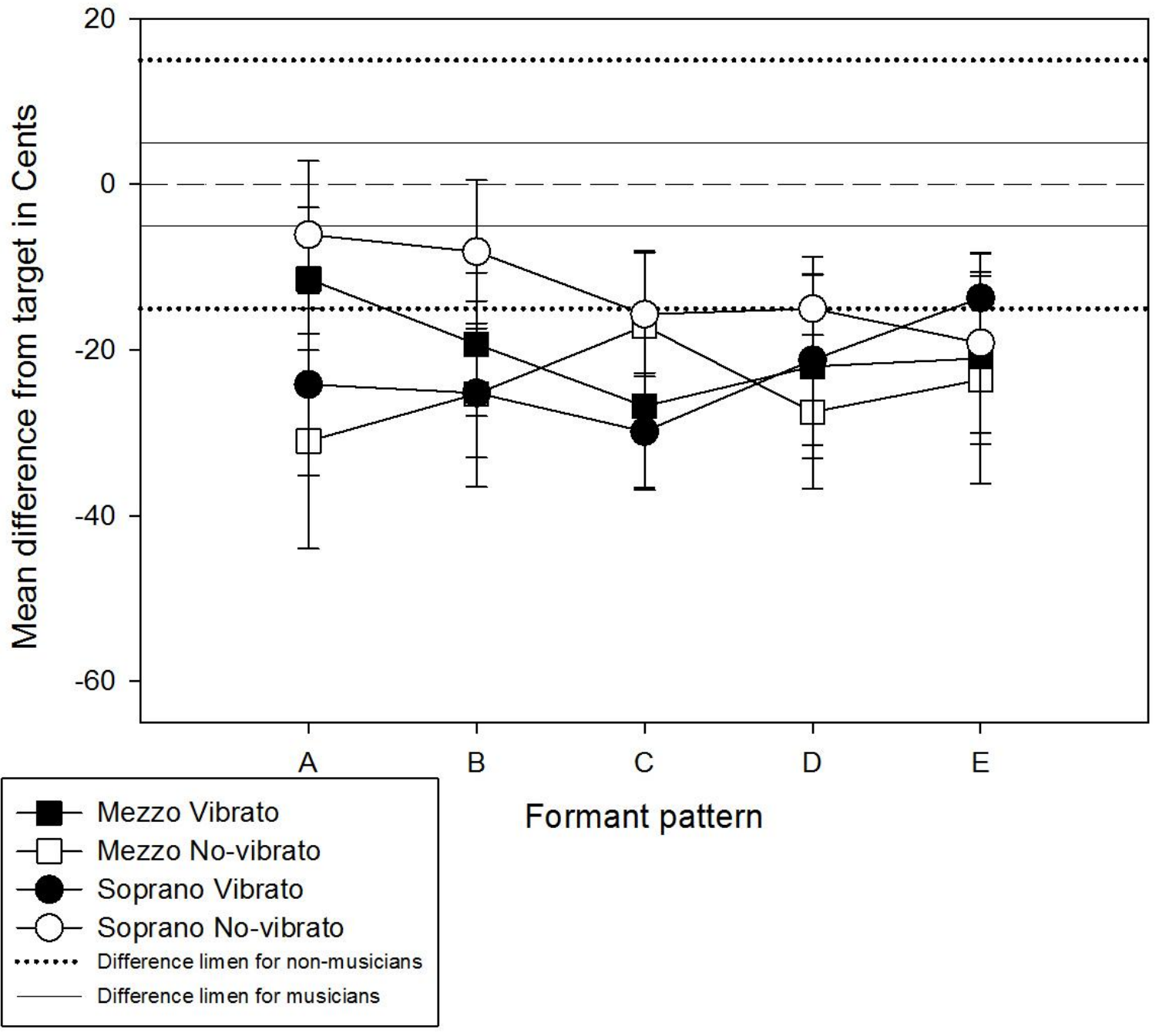

Figure 10. Mean difference in cents between the target and the experimental productions as a function of formant pattern and vibrato at pitch $F 5$ in prephonatory condition. 
Table 10. Results of $2 \times 3 \times 5 \times 2$ ANOVA for mean difference in cents between the target and the experimental productions in mid-point of the vowel condition.

\begin{tabular}{|c|c|c|c|c|}
\hline Effect & $\mathrm{F}$ & Hypotheses df & Error df & Sig. \\
\hline Category & .498 & 1 & 21 & .488 \\
\hline Pitch & 20.360 & 2 & 42 & $<.001 *$ \\
\hline Pitch*category & .339 & 2 & 42 & .714 \\
\hline Pattern & .473 & 4 & 84 & .756 \\
\hline Pattern*category & .797 & 4 & 84 & .531 \\
\hline Vibrato & .453 & 1 & 21 & .508 \\
\hline Vibrato*category & .051 & 1 & 21 & .824 \\
\hline Pitch pattern & 1.793 & 8 & 168 & .081 \\
\hline Pitch*pattern*category & .323 & 8 & 168 & .956 \\
\hline Pitch*vibrato & 5.507 & 2 & 42 & $.008^{*}$ \\
\hline Pitch*vibrato*category & .236 & 2 & 42 & .791 \\
\hline Pattern*vibrato & .167 & 4 & 84 & .955 \\
\hline Pattern*vibrato*category & .199 & 4 & 84 & .938 \\
\hline Pitch*pattern*vibrato & 1.174 & 8 & 168 & .318 \\
\hline Pitch*pattern*vibrato*category & .869 & 8 & 168 & .544 \\
\hline
\end{tabular}

*= significance at Alpha 0.025 after Bonferroni correction

$\mathrm{F}=$ between-groups/within-groups variance

$\mathrm{df}=$ degrees of freedom 


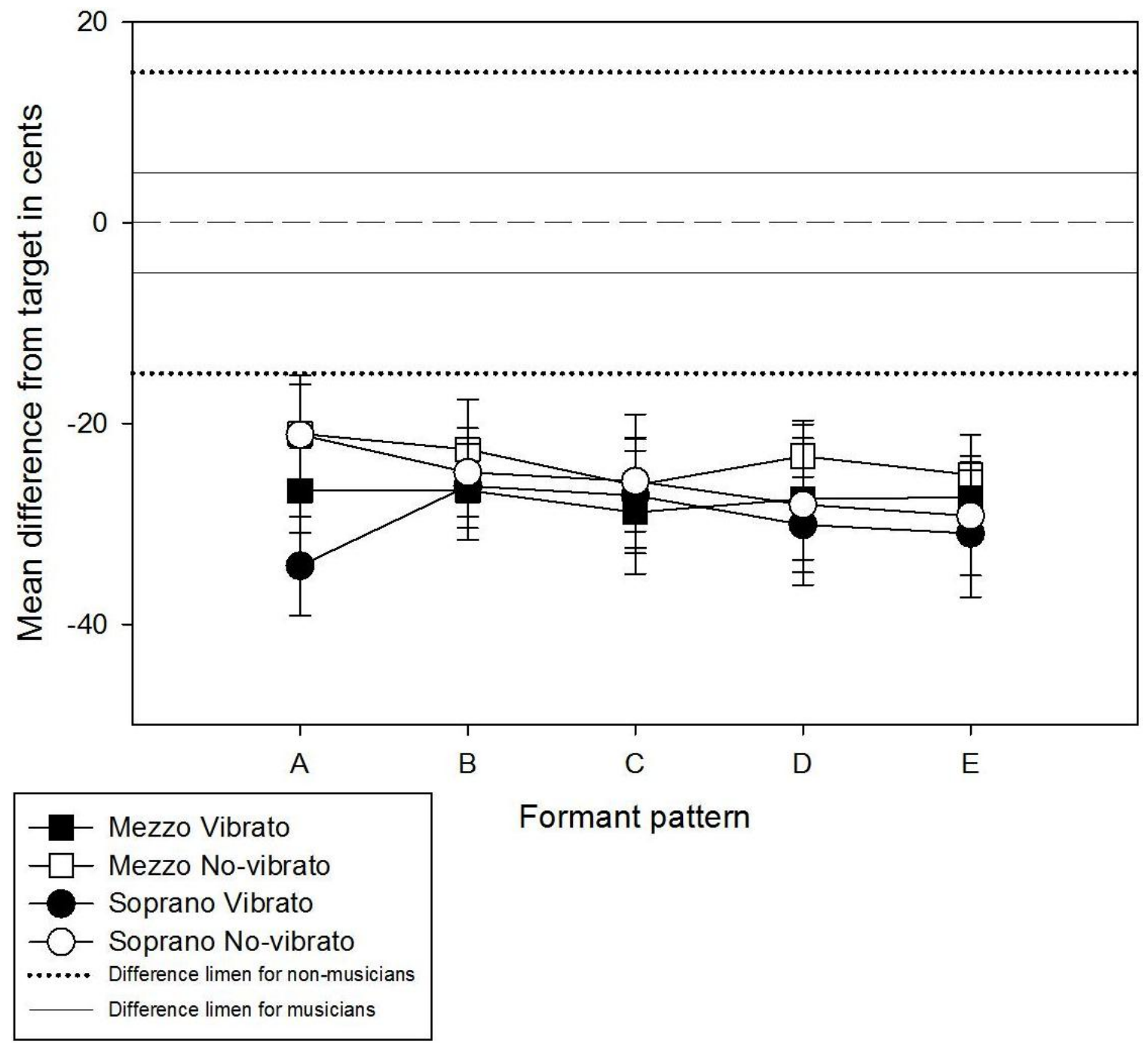

Figure 11. Mean difference in cents between the target and the experimental productions as a function of formant pattern and vibrato at pitch $\mathrm{C} 4 \mathrm{in}$ mid-point of the vowel condition. 


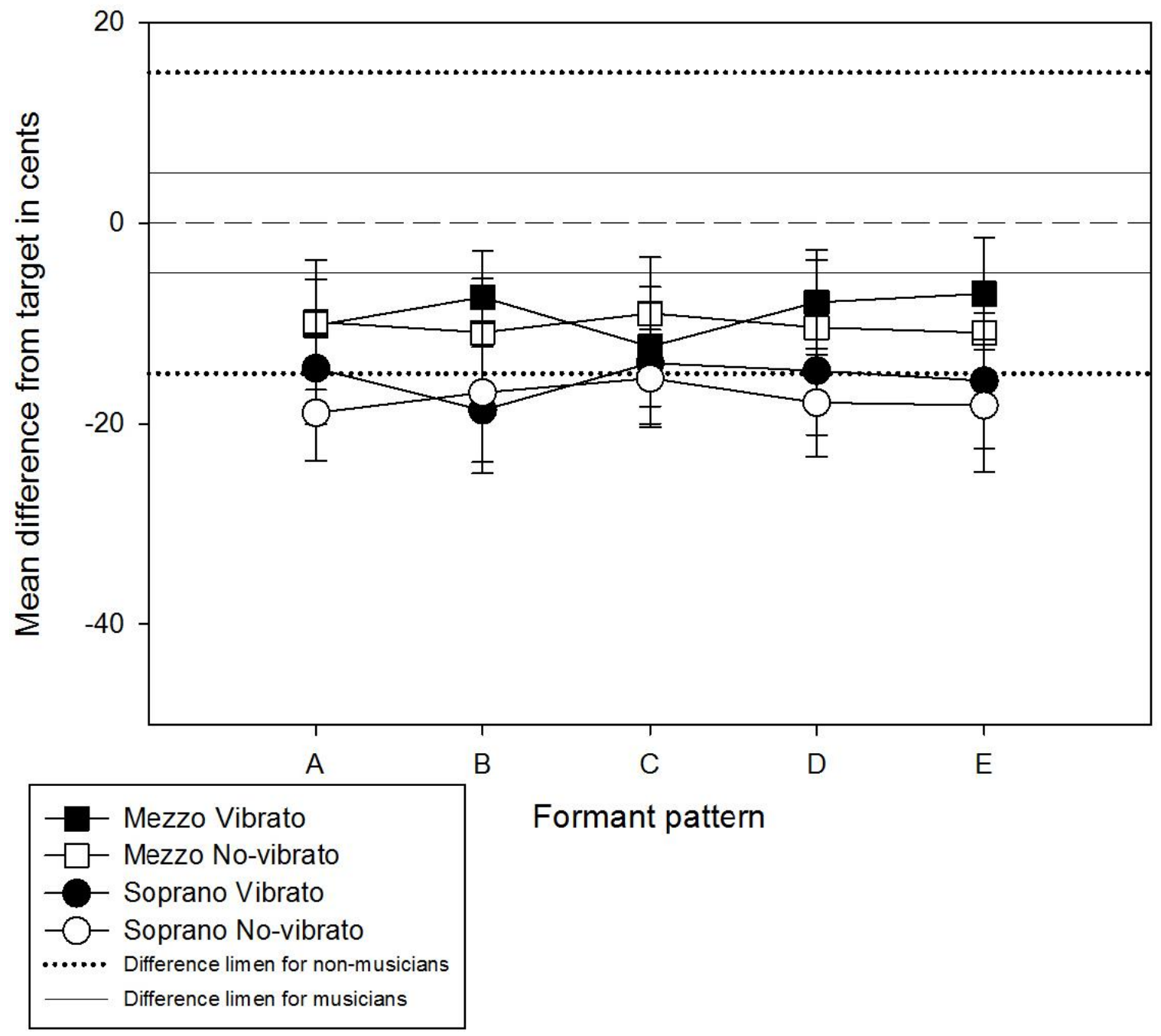

Figure 12. Mean difference in cents between the target and the experimental productions as a function of formant pattern and vibrato at pitch B4 in mid-point of the vowel condition. 


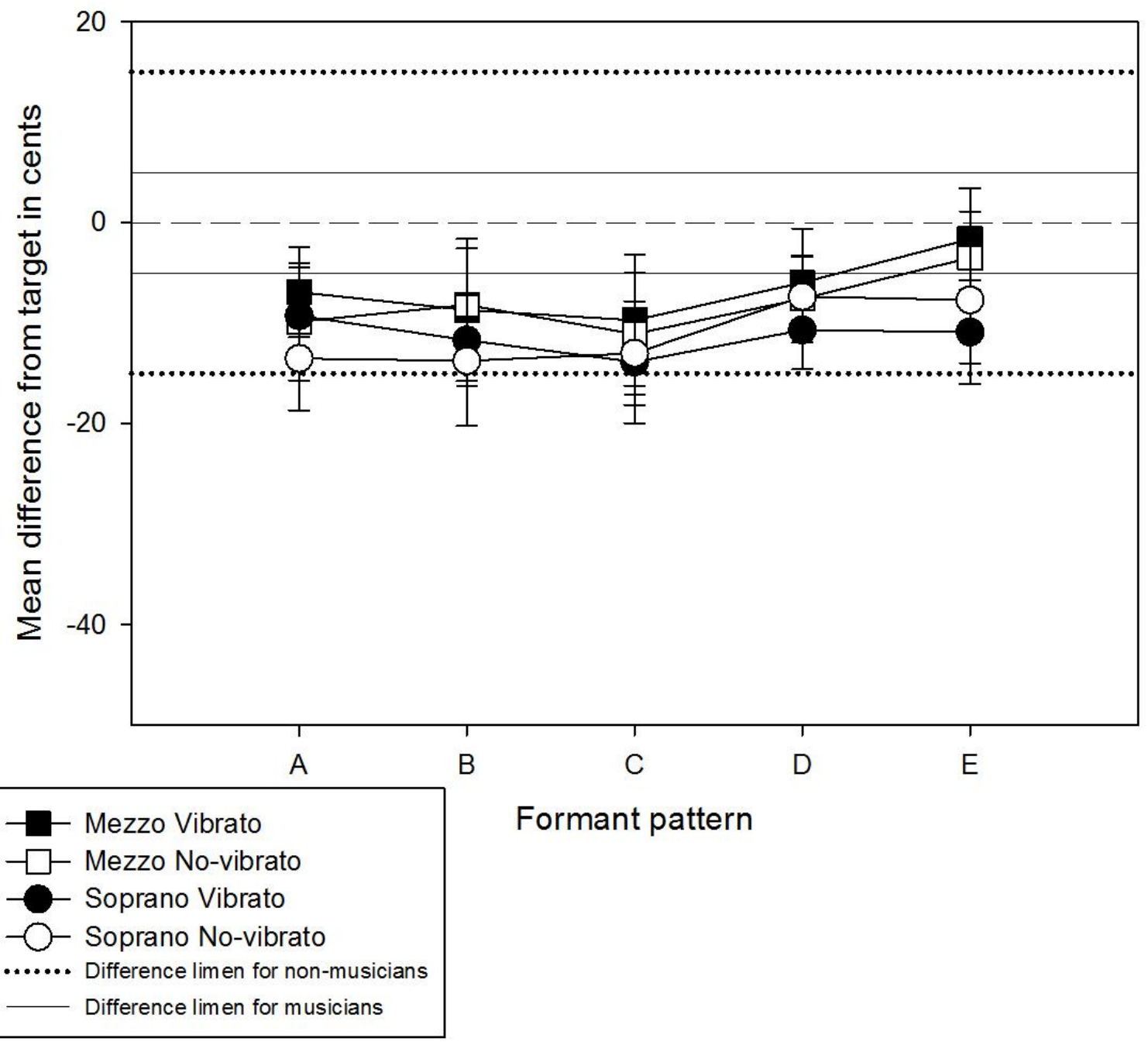

Figure 13. Mean difference in cents between the target and the experimental productions as a function of formant pattern and vibrato at pitch $\mathrm{F5}$ in mid-point of the vowel condition. 
Post-hoc ANOVAs were performed to understand the effect of pitch on the mean difference in cents between the target and the experimental productions in the vibrato and no-vibrato conditions. There was a significant main effect of pitch on the mean difference in cents between the target and the experimental productions in both vibrato $\left(\mathrm{F}_{(2,42)}\right.$ $=23.685, \mathrm{P}<.001)$ and no-vibrato conditions $\left(\mathrm{F}_{(2,42)}=14.125, \mathrm{P}<.001\right)$. Results of posthoc ANOVAs for mean difference in cents between target and experimental productions at vibrato and no-vibrato conditions are presented in the Tables 11 and 12. Mean difference in cents between the target and the experimental productions for mezzosopranos and sopranos in vibrato condition is shown in the Figures 14-16 for pitches C4, B4, and F5, respectively. Mean difference in cents between the target and the experimental productions for mezzo-sopranos and sopranos in the no-vibrato condition is shown in the Figures 17-19 for pitches C4, B4, and F5, respectively.

Tests of within-subjects contrasts indicate that there is a significant quadratic relationship between the pitch and mean difference in cents at both vibrato $\left(\mathrm{F}_{(1,21)}=\right.$ $17.669, p<.001)$ as well as no-vibrato $\left(\mathrm{F}_{(1,21)}=33.730, p<.001\right)$ conditions. Mean difference averaged over all conditions as a function of pitch is presented in Figure 20. A trend towards greater accuracy with increasing pitch can be seen. The scale of X-axis has been modified to reflect the actual number of semitones between the 3 pitches, revealing a less quadratic, more linear, relationship.

Post-hoc ANOVAs were also performed to understand the effect of vibrato on the mean difference in cents between the target and the experimental productions at each of the three pitches, C4, B4, and F5. The results revealed that there was no significant main effect of vibrato at any three pitches. Likewise, no significant interactions were found.

\section{Difference between Pre-phonatory and Mid-point of the Vowel Condition}

The results of $2 \times 2 \times 3 \times 5$ ANOVA for mean difference in cents between the prephonatory and mid-point of the vowel condition is given in Table 13. A significant difference between mezzo-sopranos and sopranos was observed $\left(\mathrm{F}_{(1,21)}=7.113, p=\right.$ $.014)$. A significant effect of pitch was observed $\left(\mathrm{F}_{(2,42)}=7.451, P=.002\right)$. However, the interaction between pitch, pattern, and vibrato was also found to be significant $\left(\mathrm{F}_{(8,168)}=\right.$ $2.133), P=.035)$. No significant main effects of pattern or vibrato were seen. Mean difference in cents between the pre-phonatory and the mid-point of the vowel as a function of formant pattern and vibrato is graphically represented in Figures 21-23 for $\mathrm{C} 4, \mathrm{~B} 4$, and F5, respectively.

In the Figures 21-23, formant pattern is represented on the $\mathrm{X}$ axis and mean difference in cents between pre-phonatory and mid-point of the vowel conditions is represented on the $\mathrm{Y}$-axis. A value of zero on the $\mathrm{Y}$ axis implies that there was no difference between the pre-phonatory and mid-point of the vowel measurements. A negative value on the $\mathrm{Y}$ axis implies that the mean difference in cents between the target stimuli and the experimental production was lower in mid-point of the vowel condition than the pre-phonatory condition. A positive value on the $\mathrm{Y}$-axis implies that the mean 
Table 11. Results post-hoc ANOVA for mean difference in cents as a function of pitch and pattern in vibrato condition for the mid-point of the vowel.

\begin{tabular}{lcccc}
\hline Effect & F & Hypotheses df & Error df & Sig. \\
\hline Category & .537 & 1 & 21 & .472 \\
Pitch & 23.685 & 2 & 42 & $<.001^{*}$ \\
Pitch*category & .242 & 2 & 42 & .786 \\
Pattern & .607 & 4 & 84 & .658 \\
Pattern*category & 1.060 & 4 & 84 & .382 \\
Pitch pattern & .830 & 8 & 168 & .578 \\
Pitch*pattern*category & .801 & 8 & 168 & .602 \\
\hline
\end{tabular}

*= significance at alpha 0.012 after Bonferroni correction

$\mathrm{F}=$ between-groups/within-groups variance

$\mathrm{df}=$ degrees of freedom

Table 12. Results post-hoc ANOVA for mean difference in cents as a function of pitch and pattern in no-vibrato condition for the mid-point of the vowel.

\begin{tabular}{lrccc}
\hline Effect & F & Hypotheses df & Error df & Sig. \\
\hline Category & .445 & 1 & 21 & .512 \\
Pitch & 14.125 & 2 & 42 & $<.001^{*}$ \\
Pitch*category & .426 & 2 & 42 & .656 \\
Pattern & .153 & 4 & 84 & .961 \\
Pattern*category & .172 & 4 & 84 & .952 \\
Pitch*pattern & 2.197 & 8 & 168 & .030 \\
Pitch*pattern*category & .356 & 8 & 168 & .942 \\
\hline
\end{tabular}

*= significance at alpha 0.012 after Bonferroni correction

$\mathrm{F}=$ between-groups/within-groups variance

$\mathrm{df}=$ degrees of freedom 


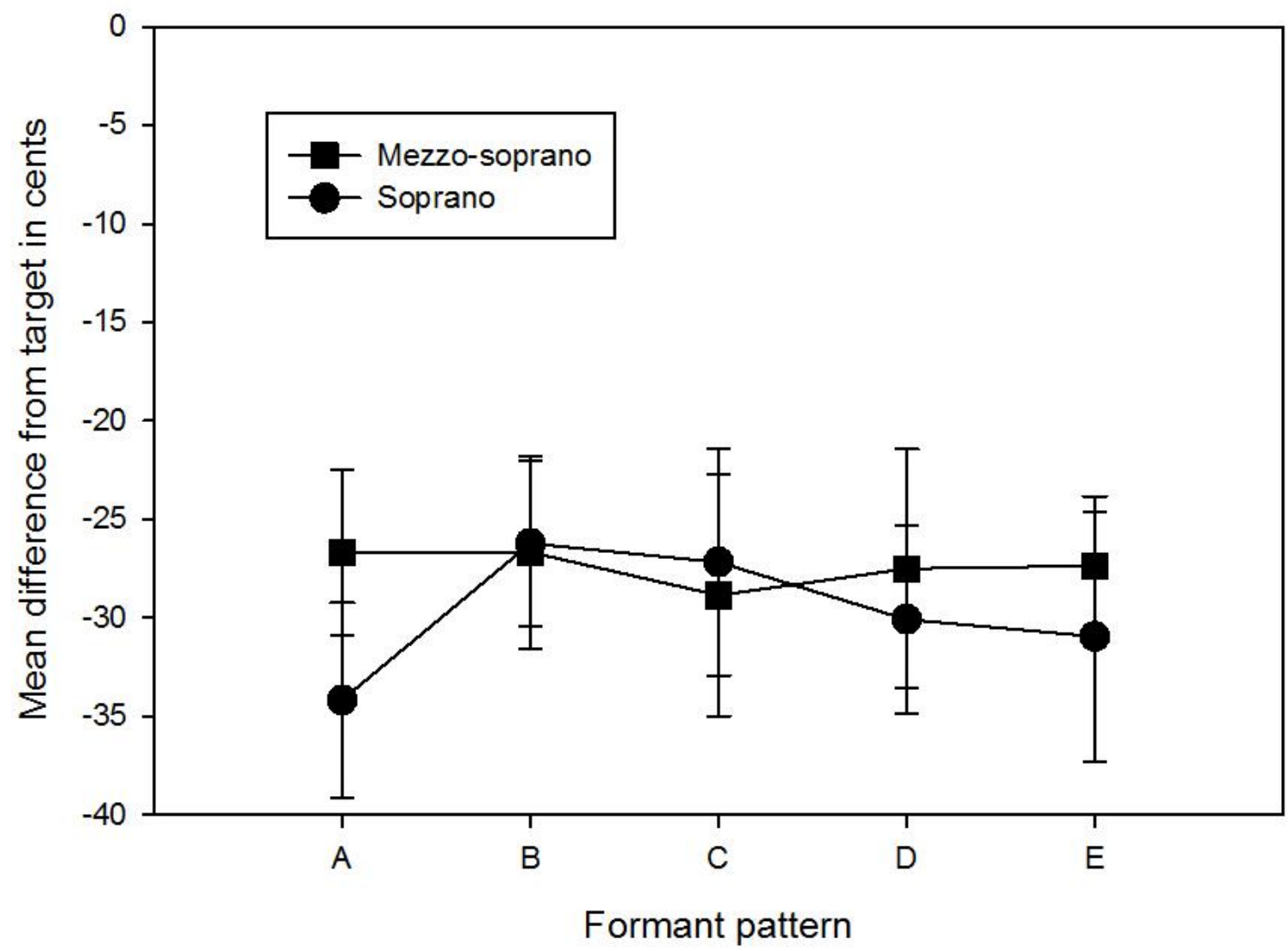

Figure 14. Mean difference in cents as a function of pitch $\mathrm{C} 4$ for mezzo-sopranos and sopranos in vibrato condition for the mid-point of the vowel. 


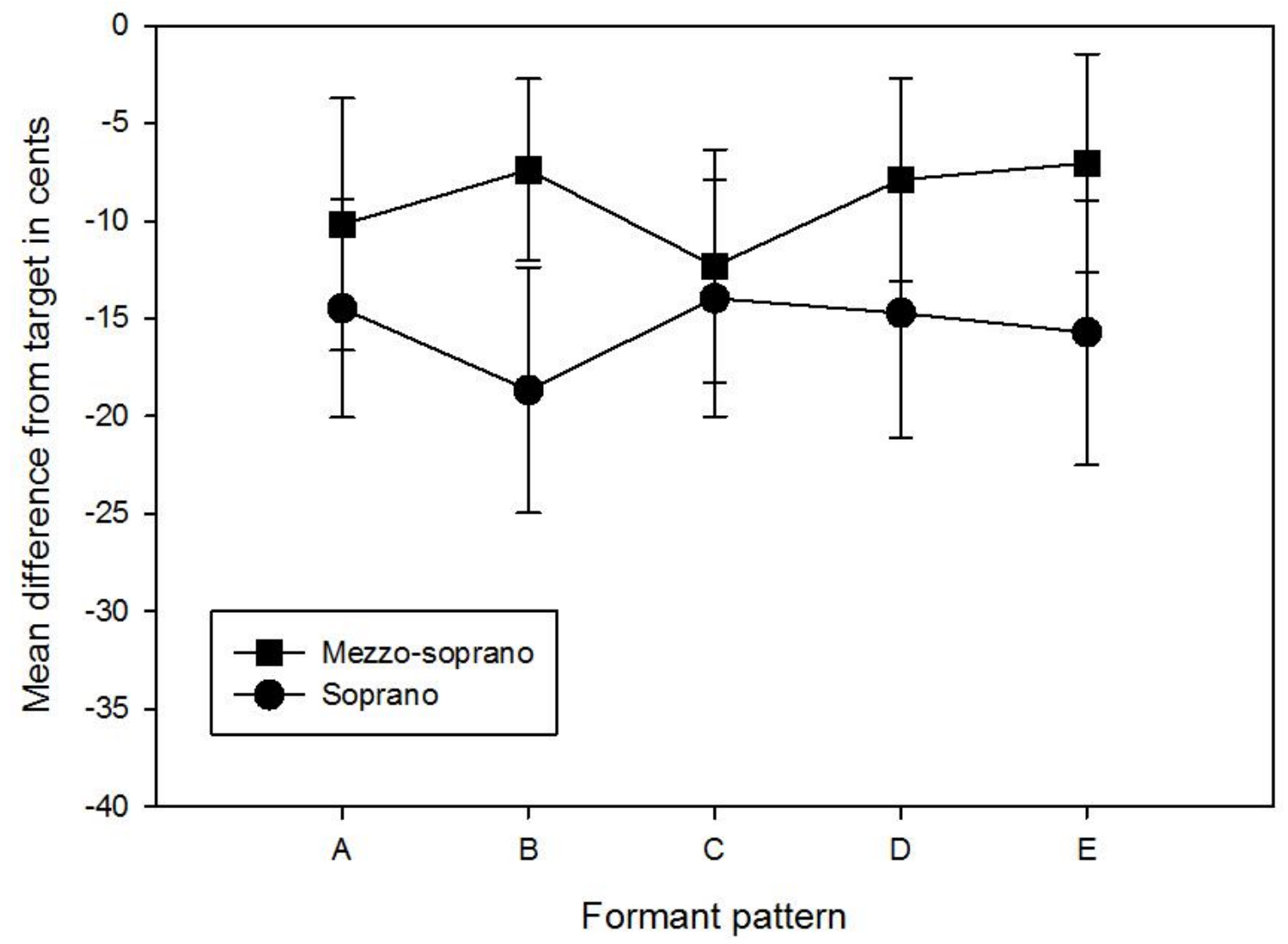

Figure 15. Mean difference in cents as a function of pitch B4 for mezzo-sopranos and sopranos in vibrato condition for the mid-point of the vowel. 


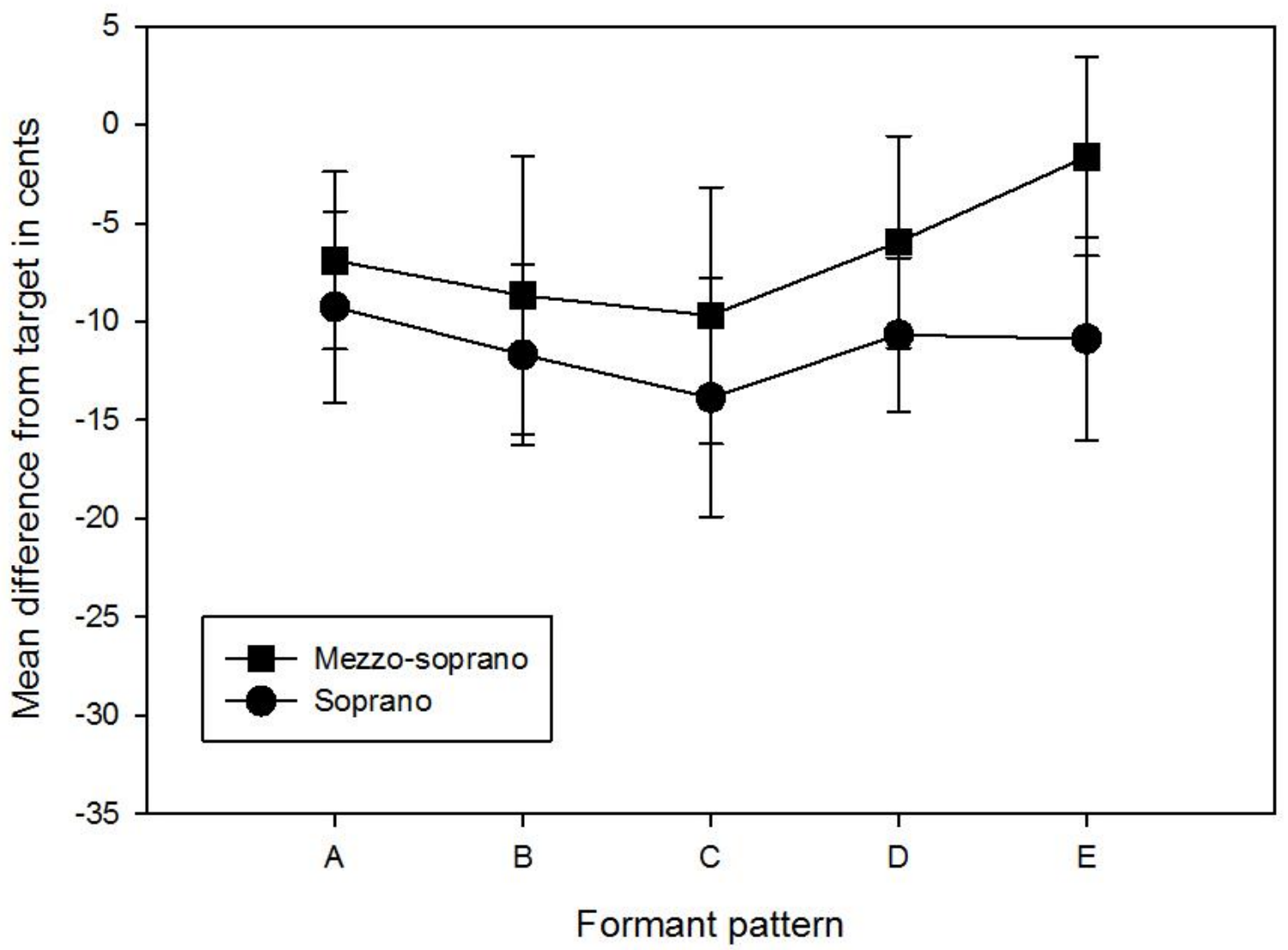

Figure 16. Mean difference in cents as a function of pitch F5 for mezzo-sopranos and sopranos in vibrato condition for the mid-point of the vowel. 


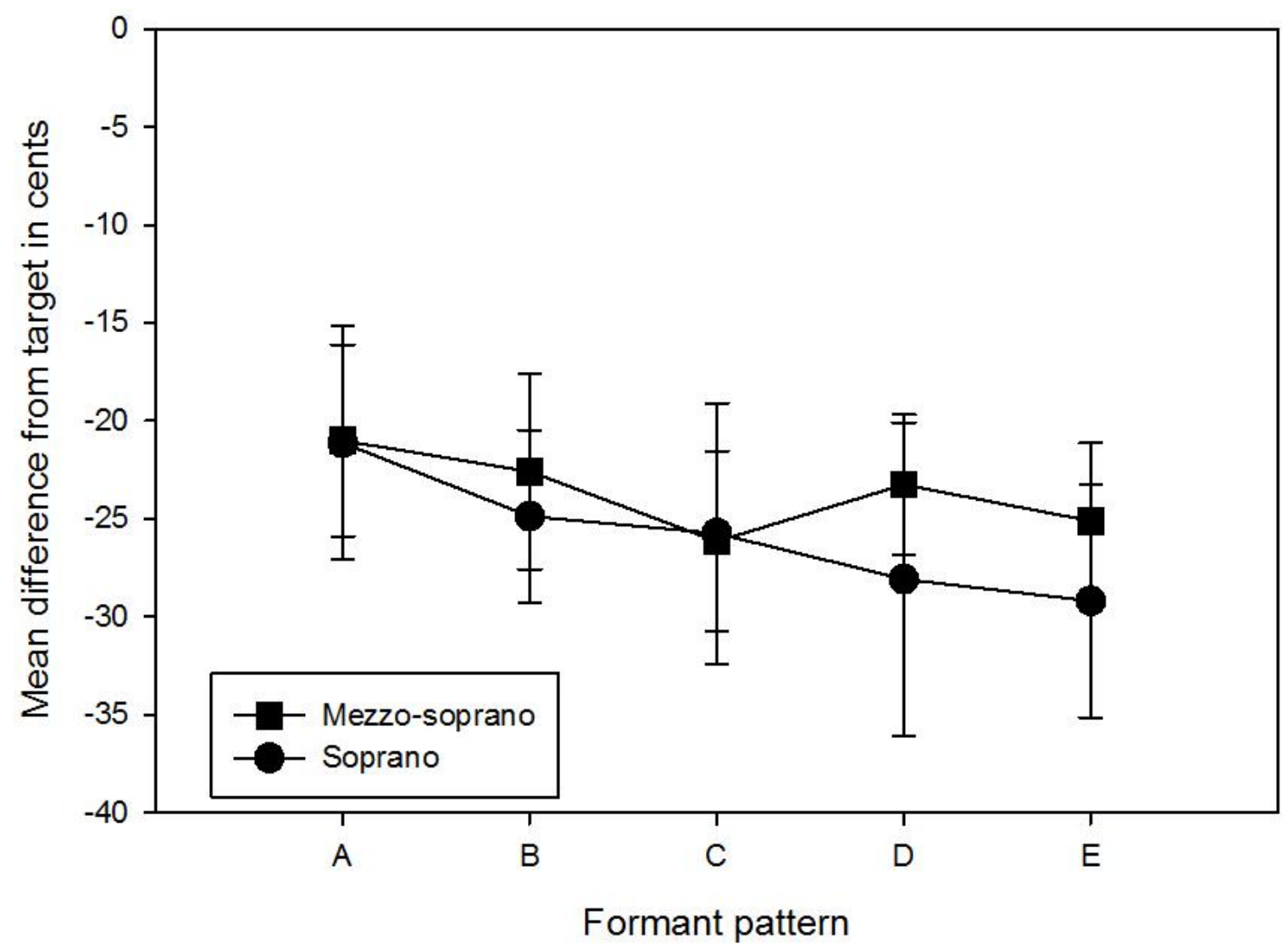

Figure 17. Mean difference in cents as a function of pitch $\mathrm{C} 4$ for mezzo-sopranos and sopranos in no-vibrato condition for the mid-point of the vowel. 


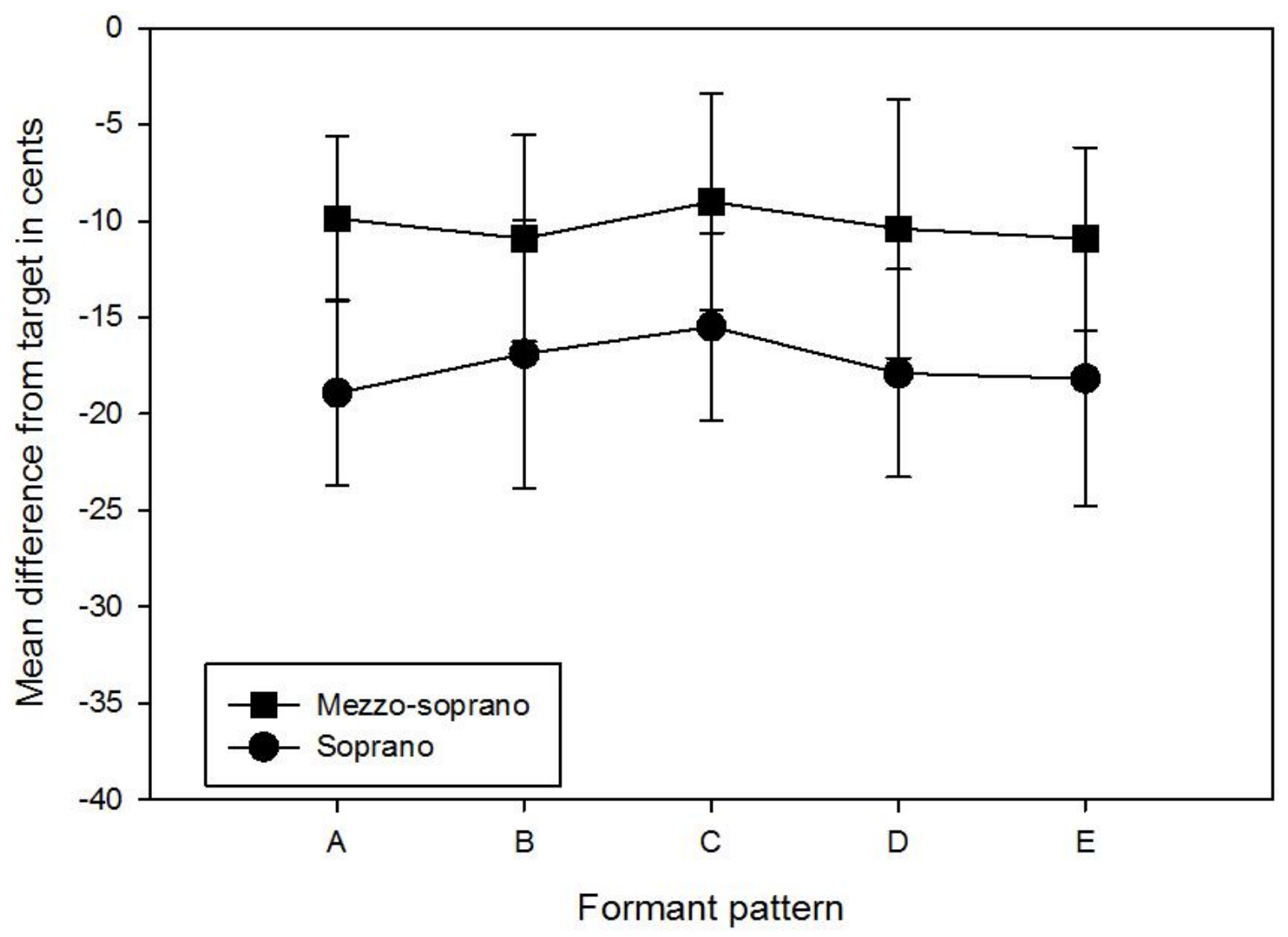

Figure 18. Mean difference in cents as a function of pitch $B 4$ for mezzo-sopranos and sopranos in no-vibrato condition for the mid-point of the vowel. 


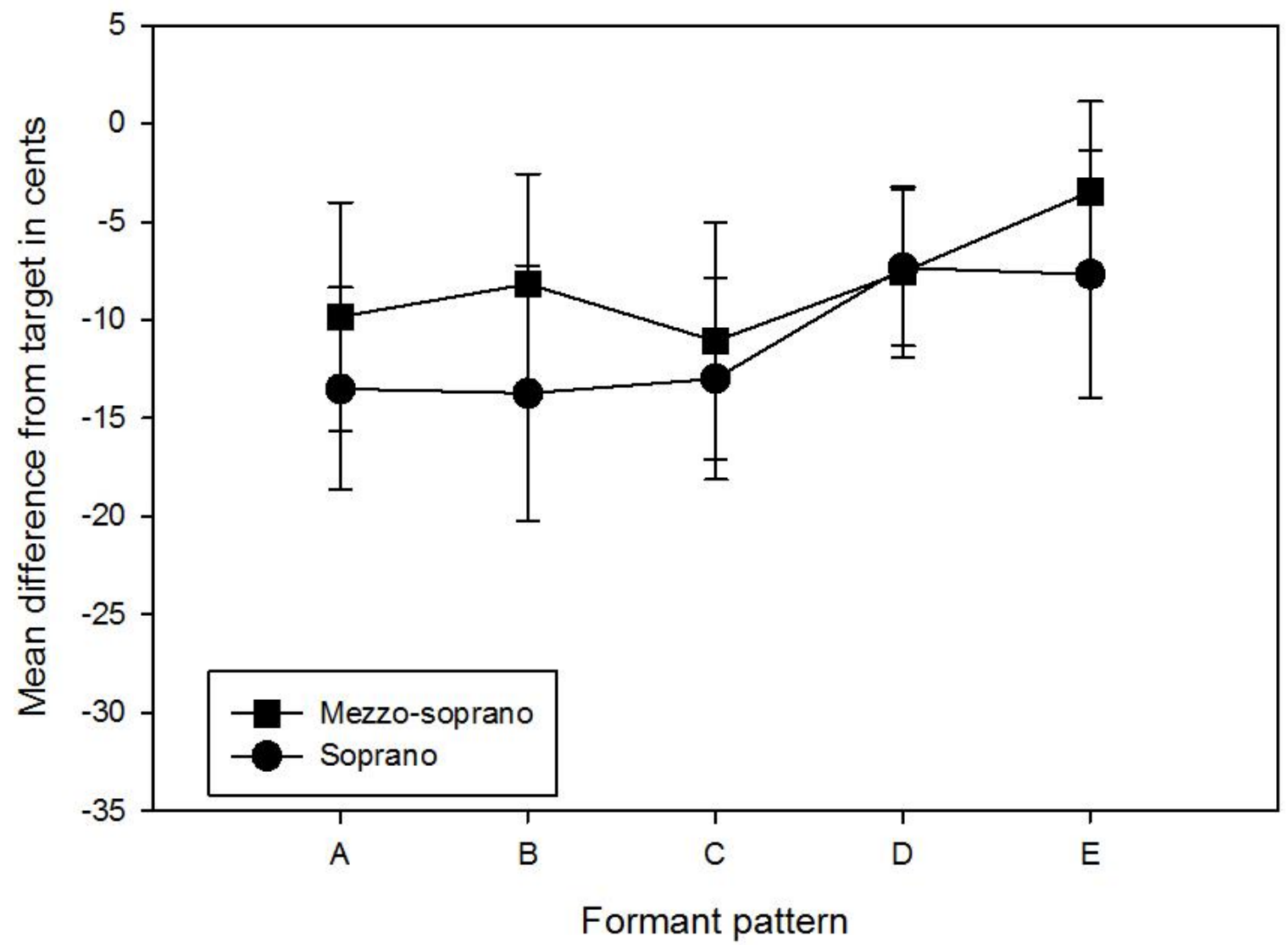

Figure 19. Mean difference in cents as a function of pitch F5 for mezzo-sopranos and sopranos in no-vibrato condition for the mid-point of the vowel. 


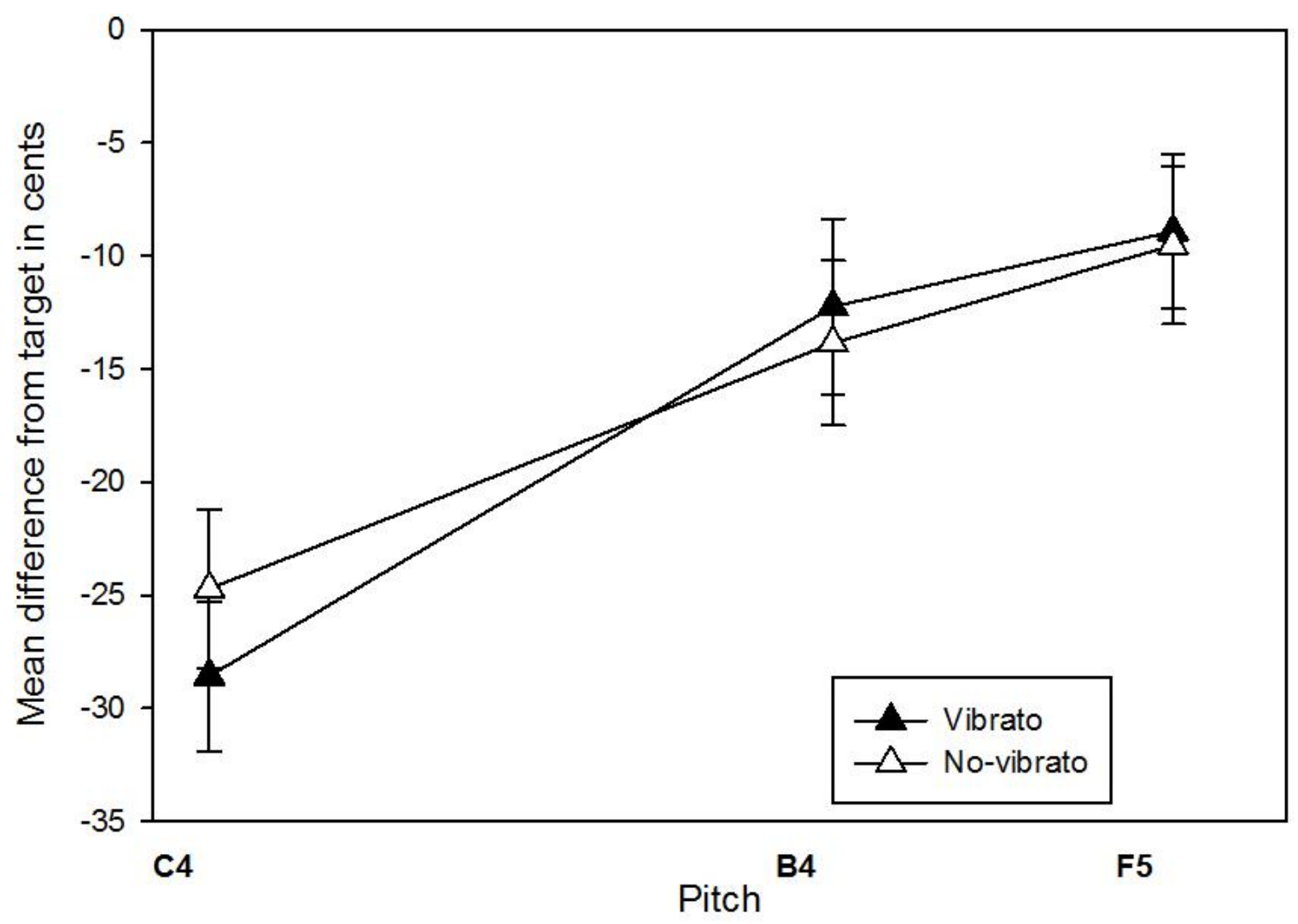

Figure 20. Mean difference in cents as a function of pitch for vibrato and novibrato conditions for the mid-point of the vowel. 
Table 13. Results of $2 \times 3 \times 5 \times 2$ ANOVA for mean difference in cents from prephonatory to mid-point of the vowel condition.

\begin{tabular}{lcccc}
\hline Effect & F & Hypotheses df & Error df & Sig. \\
\hline Category & 7.113 & 1 & 21 & $.014^{*}$ \\
Pitch & 7.451 & 2 & 42 & $.002^{*}$ \\
Pitch*category & 1.005 & 2 & 42 & .375 \\
Pattern & .551 & 4 & 84 & .699 \\
Pattern*category & 1.243 & 4 & 84 & .299 \\
Vibrato & .011 & 1 & 21 & .916 \\
Vibrato*category & .427 & 1 & 21 & .521 \\
Pitch*pattern & .993 & 8 & 168 & .444 \\
Pitch*pattern*category & 1.210 & 8 & 168 & .296 \\
Pitch*vibrato & .421 & 2 & 42 & .659 \\
Pitch*vibrato*category & .968 & 2 & 42 & .388 \\
Pattern*vibrato & .379 & 4 & 84 & .823 \\
Pattern*vibrato*category & .551 & 4 & 168 & .699 \\
Pitch*pattern*vibrato & 2.133 & 8 & .280 \\
Pitch*pattern*vibrato*category & 1.238 & 8 & & $.035^{*}$ \\
\hline
\end{tabular}

*= significance at Alpha 0.05

$\mathrm{F}=$ between-groups/within-groups variance

$\mathrm{df}=$ degrees of freedom 


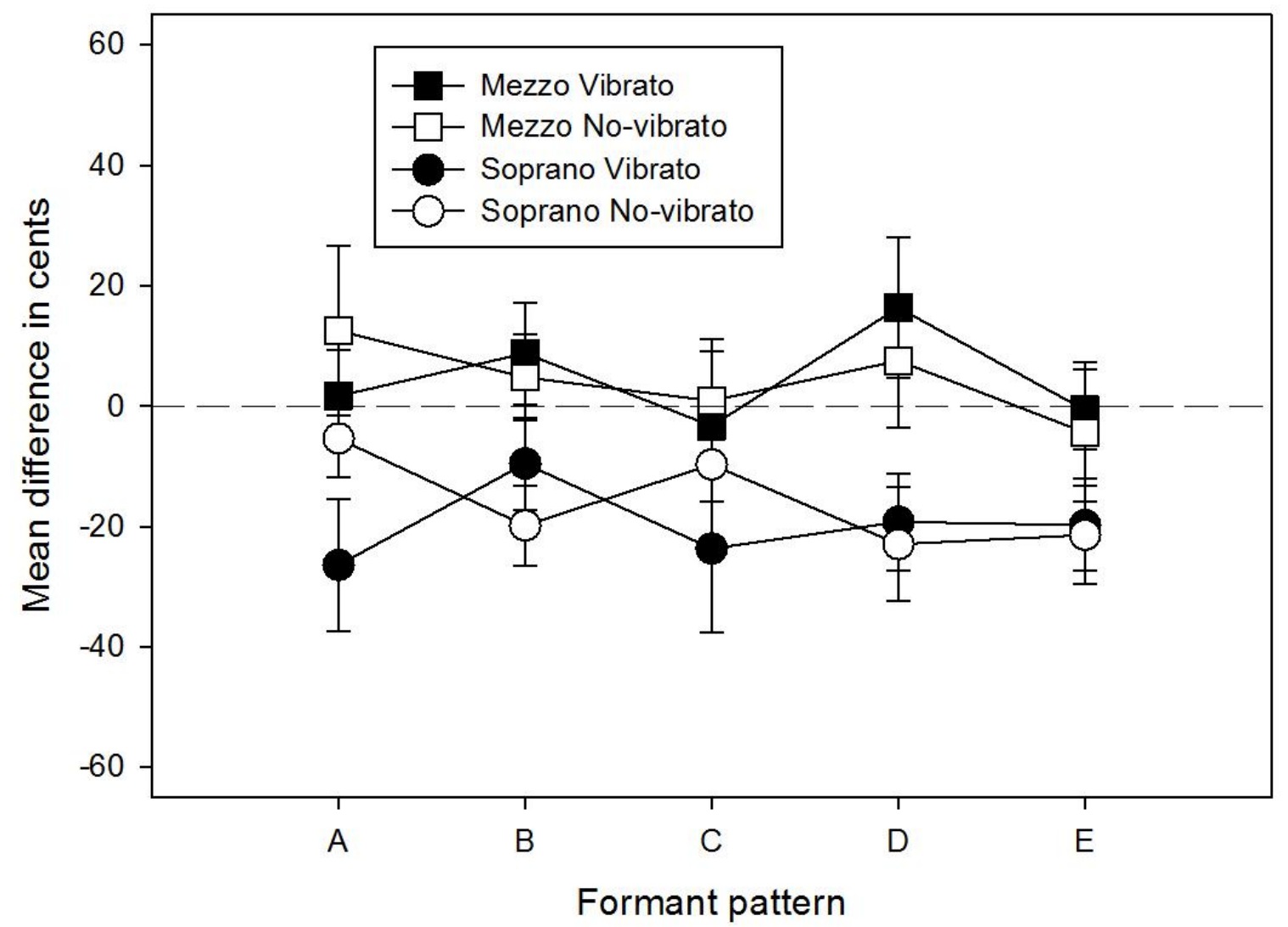

Figure 21. Mean difference in cents between pre-phonatory and mid-point of the vowel conditions as a function of formant pattern and vibrato at pitch $\mathrm{C4}$. 


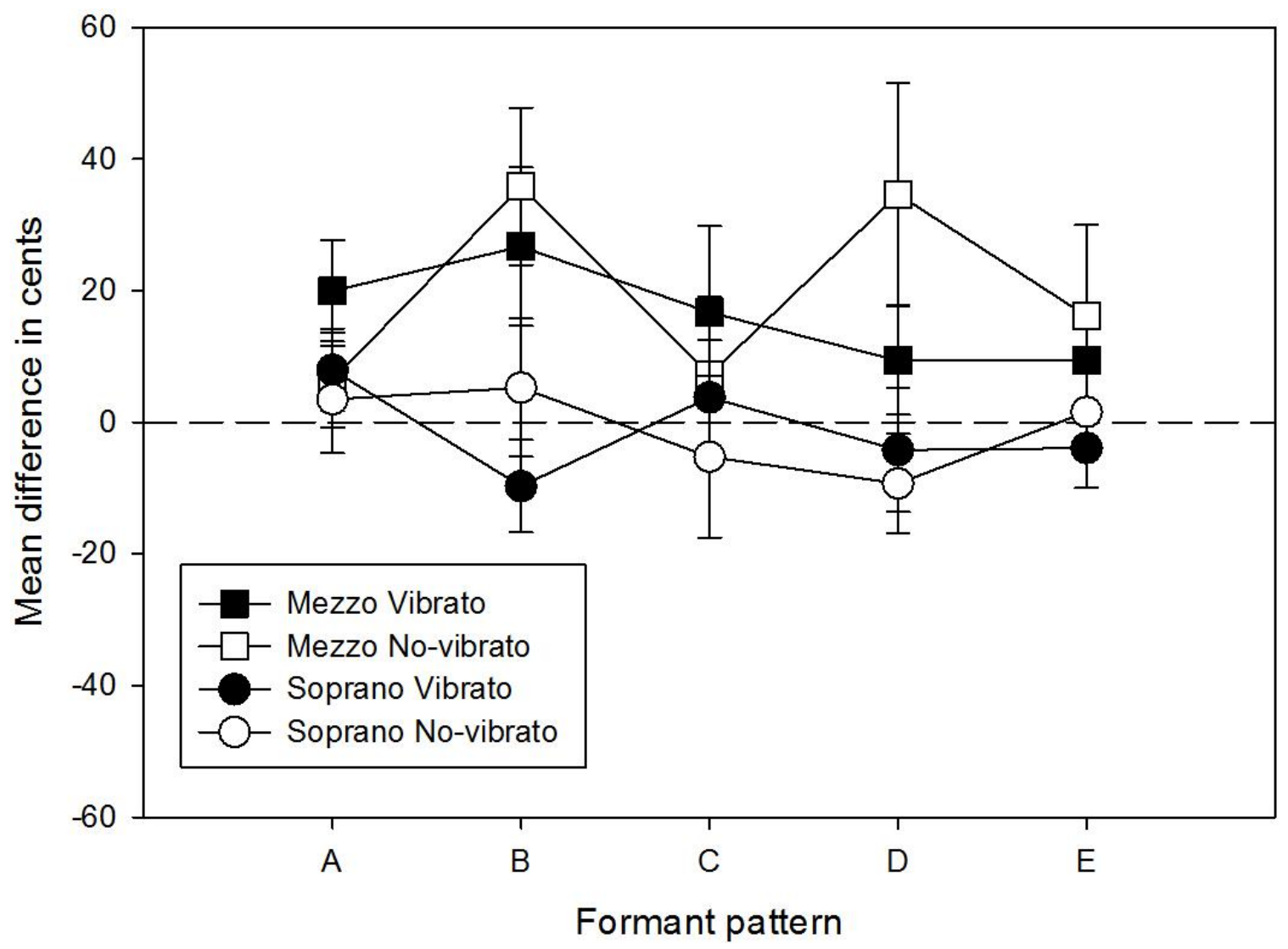

Figure 22. Mean difference in cents between pre-phonatory and mid-point of the vowel conditions as a function of formant pattern and vibrato at pitch B4. 


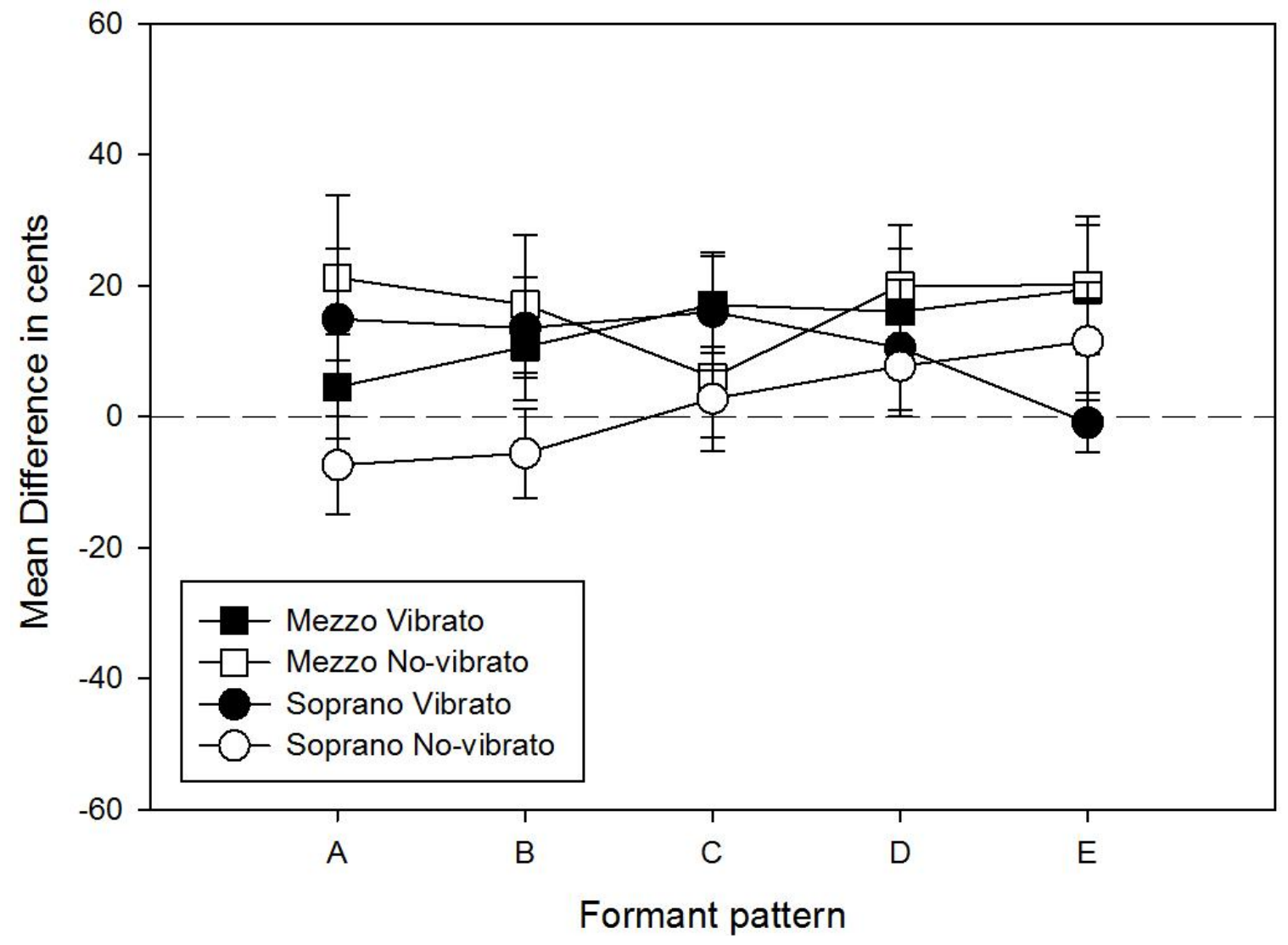

Figure 23. Mean difference in cents between pre-phonatory and mid-point of the vowel conditions as a function of formant pattern and vibrato at pitch F5. 
difference in cents between target stimuli and experimental production is higher in midpoint of the vowel condition than pre-phonatory condition.

Further post-hoc ANOVAs were conducted to investigate the effect of pitch on mean difference in cents between pre-phonatory and mid-point of the vowel points at each of vibrato and no-vibrato conditions. The results are presented in the Tables 14 and 15 for vibrato and no-vibrato conditions, respectively. The results indicate that there was a main effect of pitch on mean difference in cents between pre-phonatory and mid-point of the vowel points in both vibrato $\left(\mathrm{F}_{(2,42)}=5.241, p=.009\right)$ and no-vibrato $\left(\mathrm{F}_{(2,42)}=\right.$ $5.241, p=.005)$ conditions. Mean difference in cents between pre-phonatory and midpoint of the vowel for vibrato condition is given in the Figures 24-26 for pitches C4, B4, and F5, respectively. Mean difference in cents between pre-phonatory and mid-point of the vowel for no-vibrato condition is given in Figures 27-29 for C4, B4 and F5, respectively. Vector plots representing the magnitude and direction of change in pitch are displayed in the Figures 30-32 for vibrato condition and Figures 33-35 for no-vibrato condition, respectively.

Tests of within-subjects contrasts indicate that there is a significant quadratic relationship between pitch and mean difference in cents between pre-phonatory phase and mid-point of the vowel in both vibrato $\left(\mathrm{F}_{(1,21)}=10.590, p=.004\right)$ as well as novibrato $\left(\mathrm{F}_{(1,21)}=8.083, p=.010\right)$ conditions. The relationship between pitch and mean difference in cents between pre-phonatory phase and mid-point of the vowel is graphically presented in the Figure 36. The X-axis of Figure 36 has been scaled to accurately reflect the number of semitones between the pitches. It can be seen that greatest amount of tuning occurs at the lowest pitch $\mathrm{C} 4$. 
Table 14. Results post-hoc ANOVA for mean difference in cents from prephonatory to mid-point of the vowel as a function of pitch and pattern in vibrato condition.

\begin{tabular}{lcccc}
\hline Effect & F & Hypotheses df & Error df & Sig. \\
\hline Category & 5.944 & 1 & 21 & $.024^{*}$ \\
Pitch & 5.241 & 2 & 42 & $.009^{*}$ \\
Pitch*category & 1.496 & 2 & 42 & .236 \\
Pattern & .516 & 4 & 84 & .724 \\
Pattern* category & .396 & 4 & 84 & .811 \\
Pitch*pattern & 1.353 & 8 & 168 & .221 \\
Pitch*pattern*category & 1.500 & 8 & 168 & .161 \\
\hline
\end{tabular}

*= significance at Alpha 0.025

$\mathrm{F}=$ between-groups/within-groups variance

$\mathrm{df}=$ degrees of freedom

Table 15. Results post-hoc ANOVA for mean difference in cents from prephonatory to mid-point of the vowel as a function of pitch and pattern in no-vibrato condition.

\begin{tabular}{lcccc}
\hline Effect & F & Hypotheses df & Error df & Sig. \\
\hline Category & 5.926 & 1 & 21 & $.024^{*}$ \\
Pitch & 6.093 & 2 & 42 & $.005^{*}$ \\
Pitch*Category & .194 & 2 & 42 & .825 \\
Pattern & .445 & 4 & 84 & .776 \\
Pattern*Category & 1.309 & 4 & 84 & .273 \\
Pitch*pattern & 1.911 & 8 & 168 & .061 \\
Pitch*pattern*Category & 1.010 & 8 & 168 & .430 \\
\hline
\end{tabular}

*= significance at Alpha 0.025

$\mathrm{F}=$ between-groups/within-groups variance

$\mathrm{df}=$ degrees of freedom 


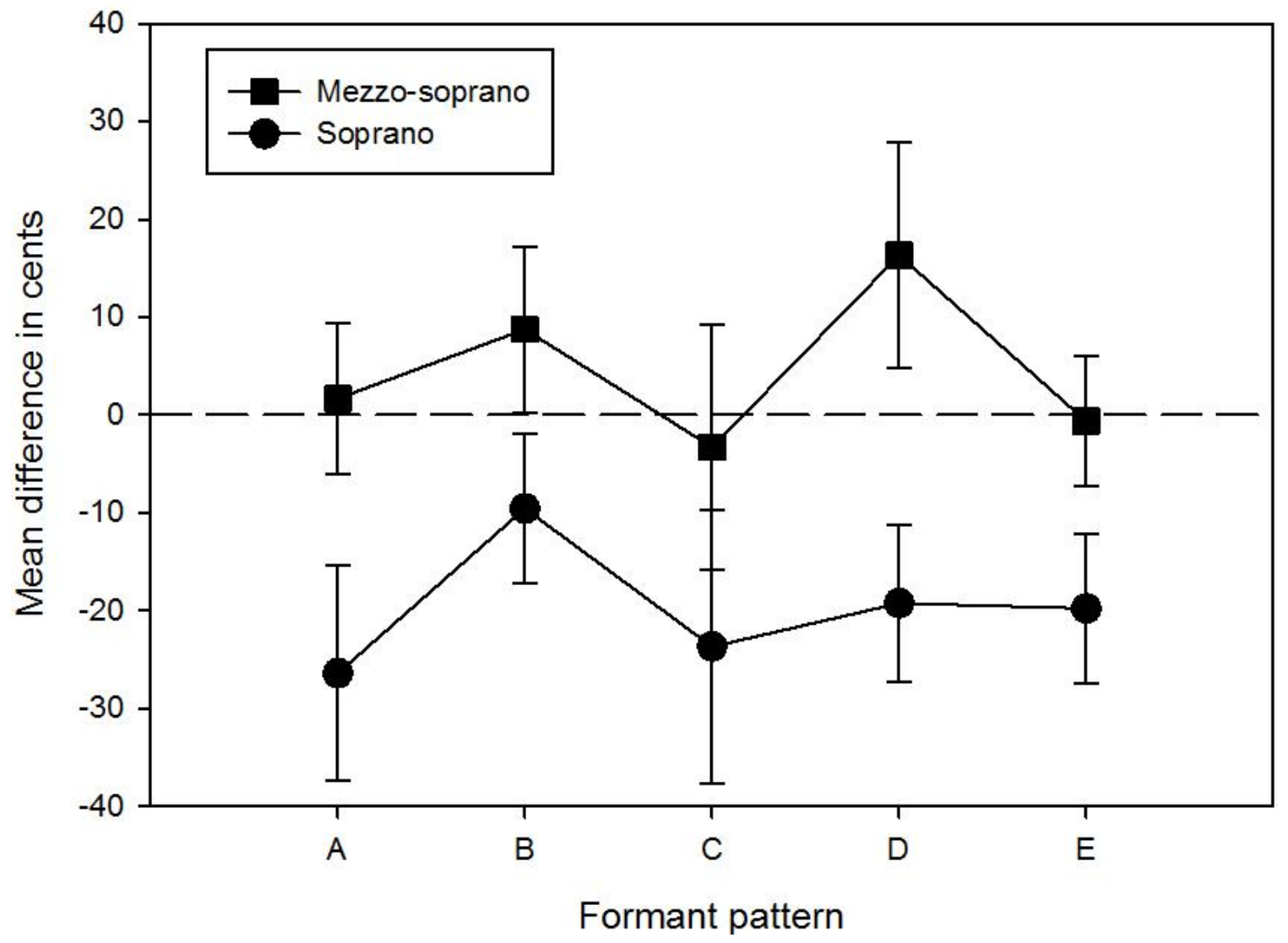

Figure 24. Mean difference in cents between pre-phonatory and mid-point of vowel in vibrato condition at pitch $\mathrm{C} 4$. 


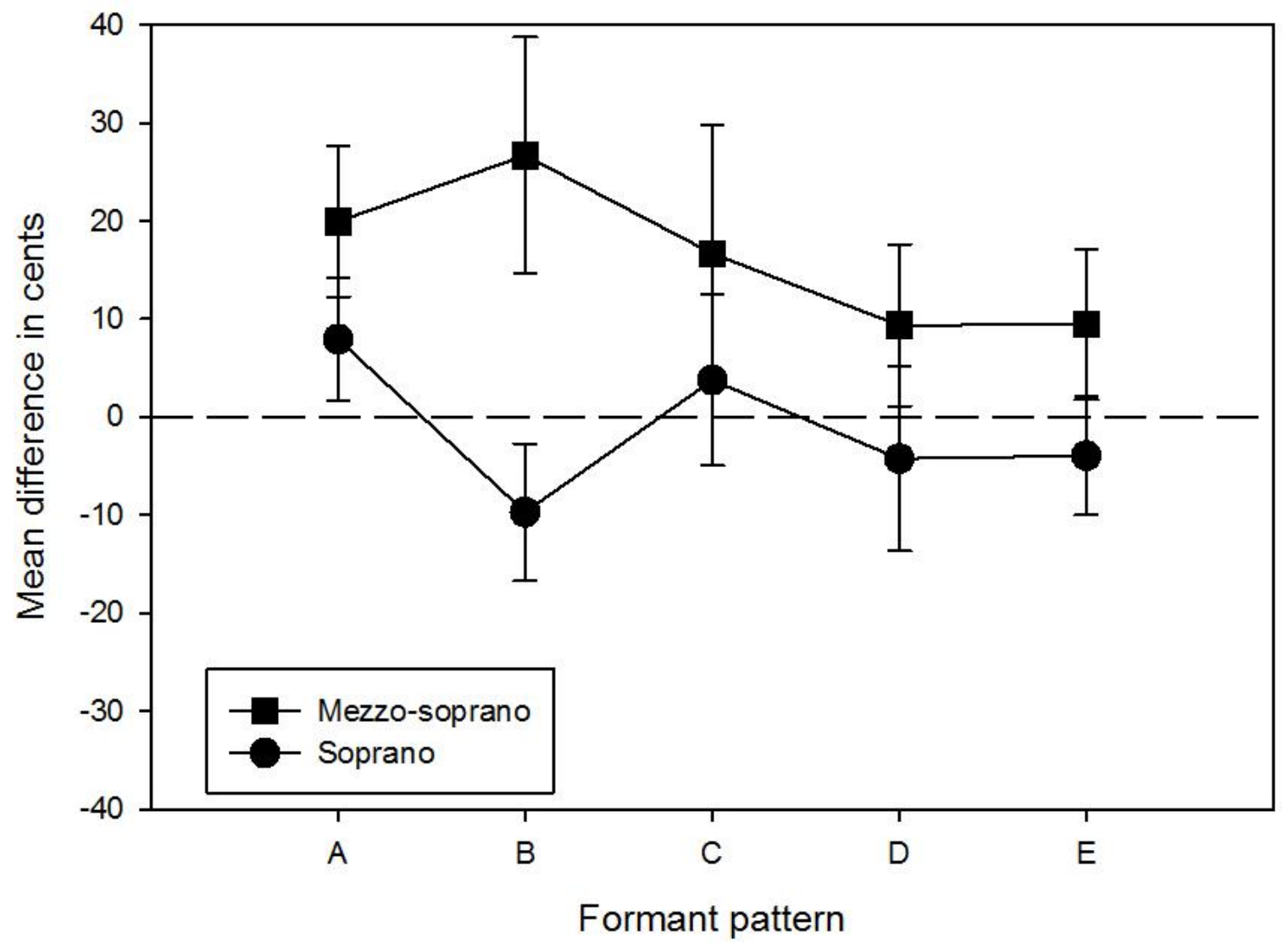

Figure 25. Mean difference in cents between pre-phonatory and mid-point of vowel in vibrato condition at pitch $B 4$. 


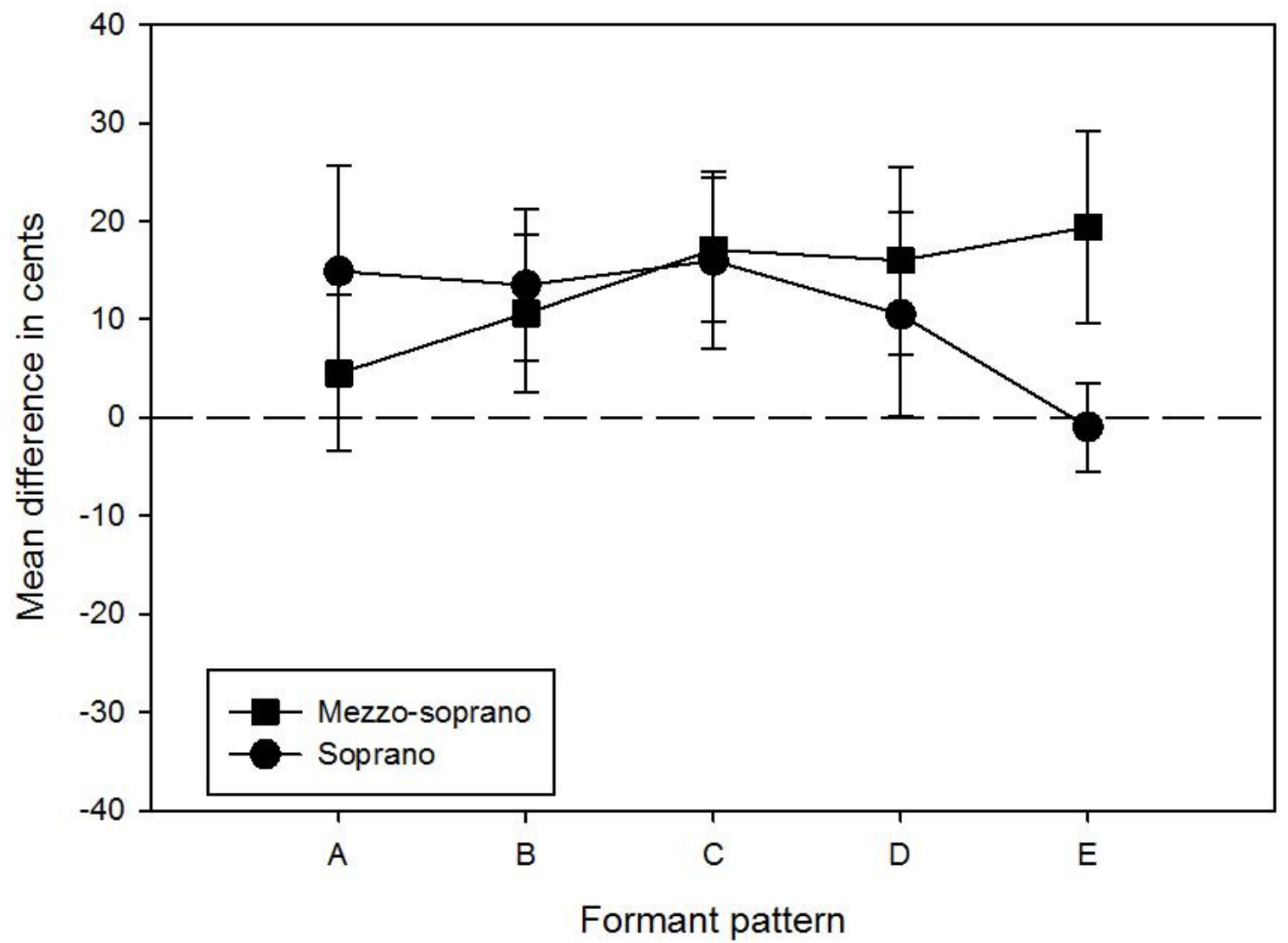

Figure 26. Mean difference in cents between pre-phonatory and mid-point of vowel in vibrato condition at pitch F5. 


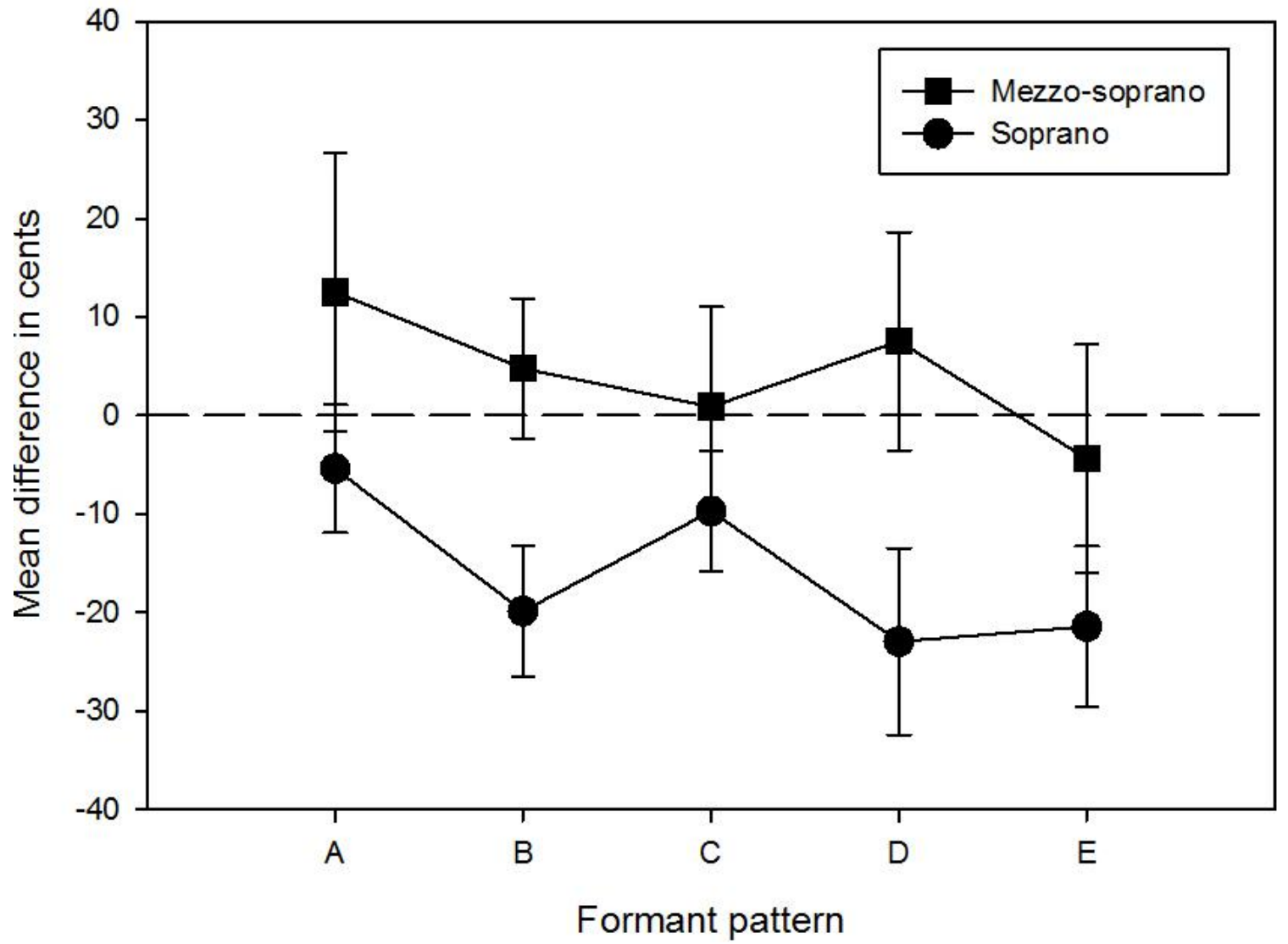

Figure 27. Mean difference in cents between pre-phonatory and mid-point of vowel in no-vibrato condition at pitch $\mathbf{C 4}$. 


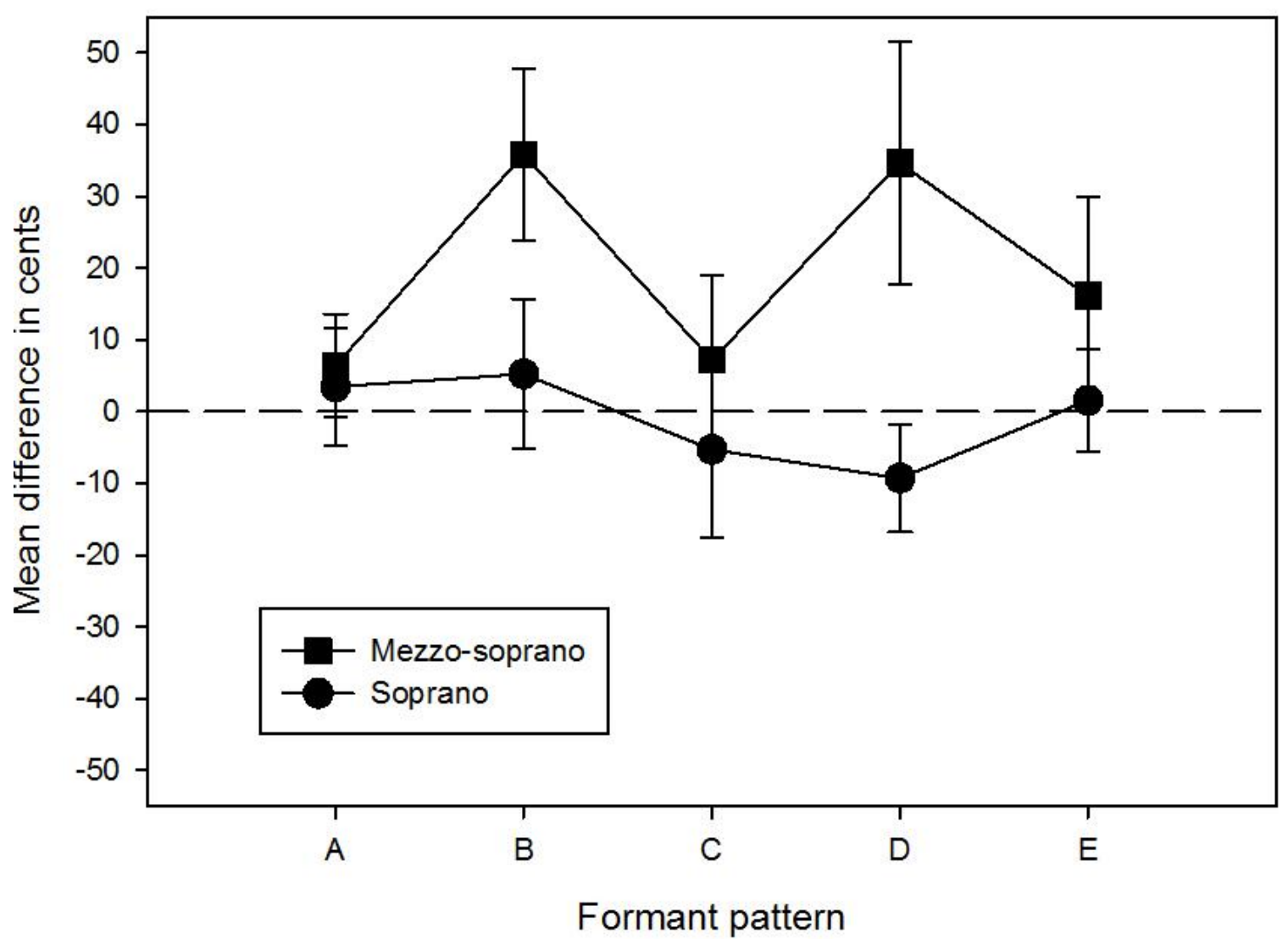

Figure 28. Mean difference in cents between pre-phonatory and mid-point of vowel in no-vibrato condition at pitch B4. 


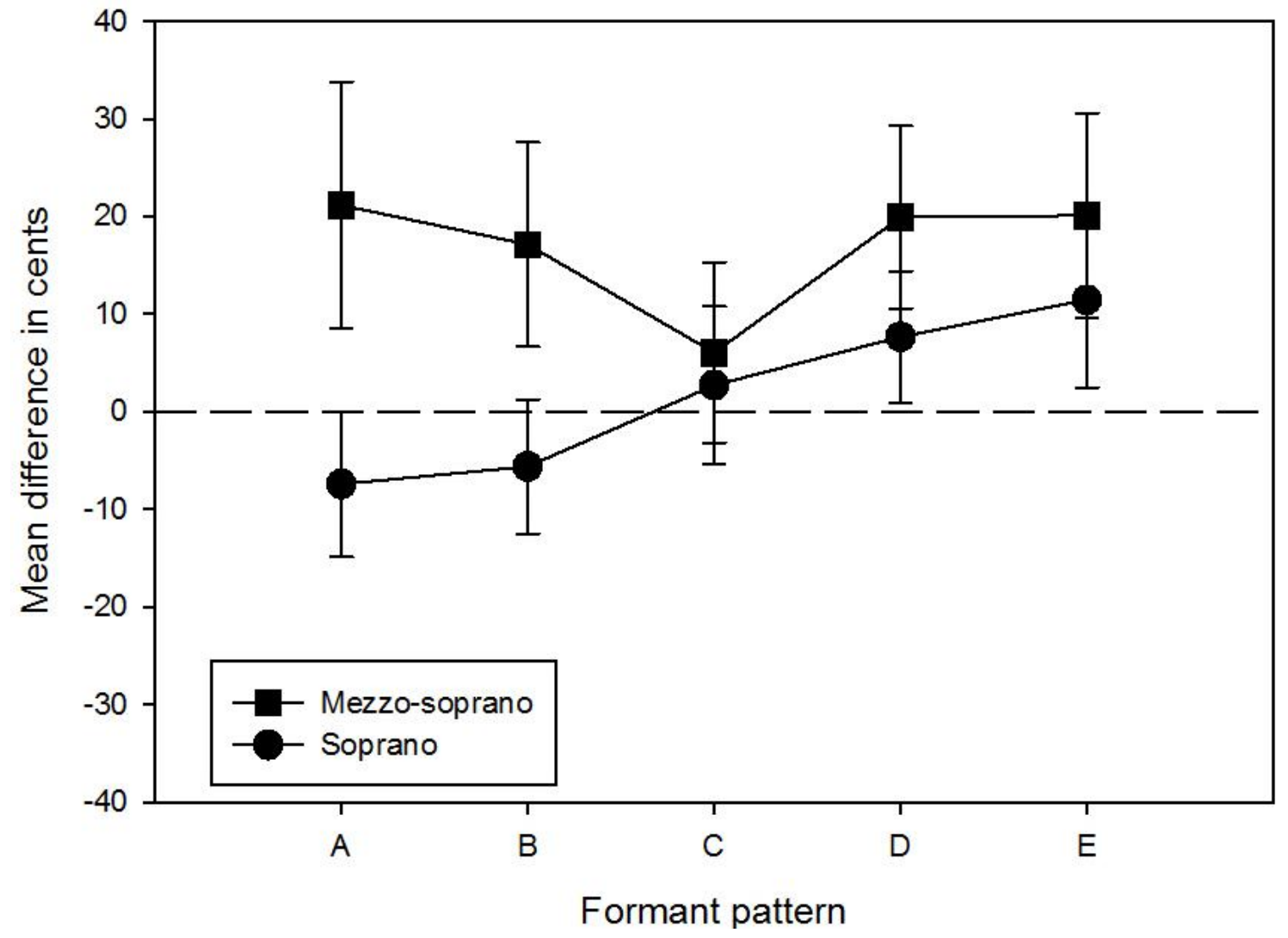

Figure 29. Mean difference in cents between pre-phonatory and mid-point of vowel in no-vibrato condition at pitch F5. 


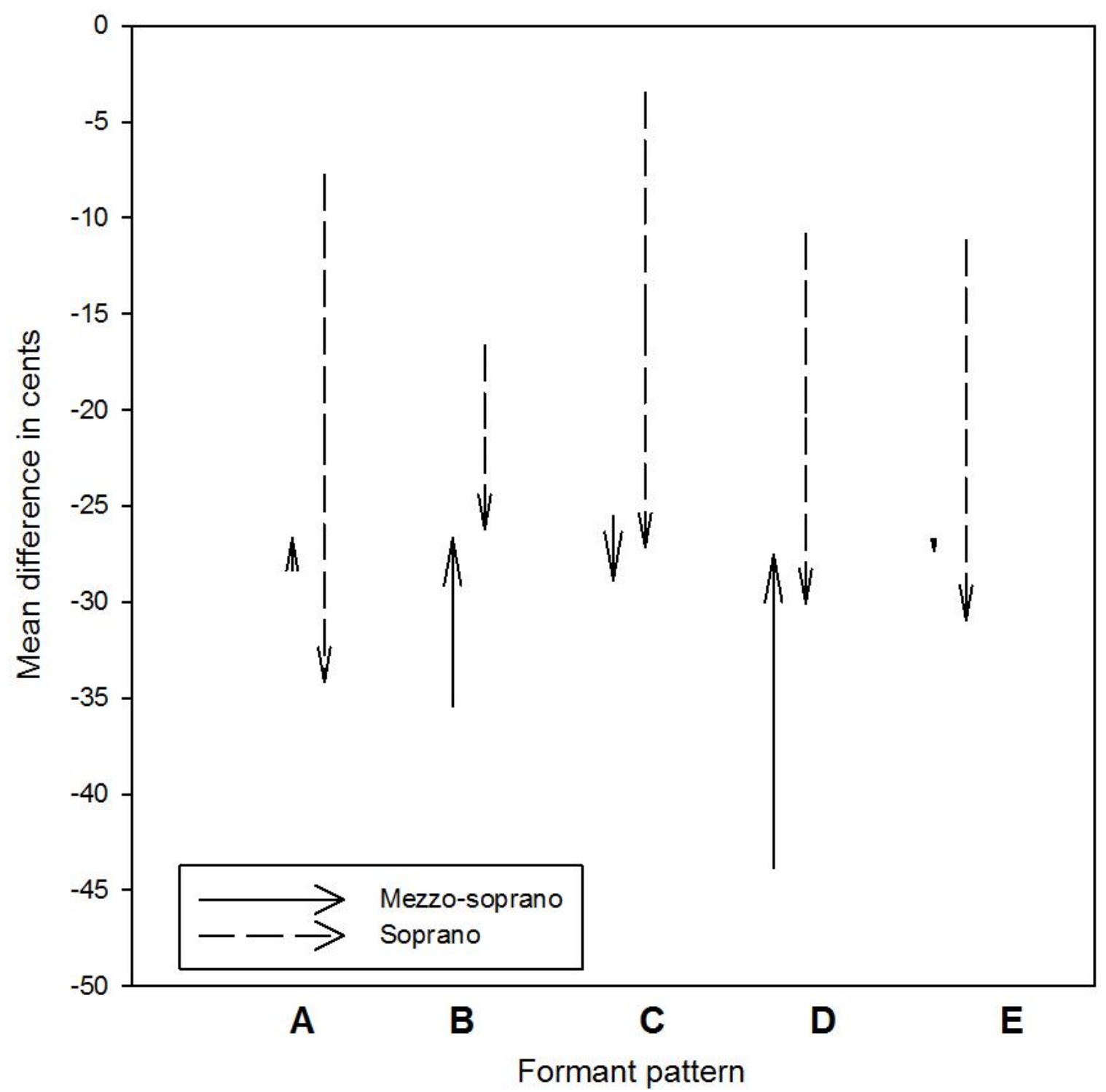

Figure 30. Vector plot representing mean difference in cents from pre-phonatory phase to mid-point of the vowel in vibrato condition at pitch $\mathrm{C} 4$. 


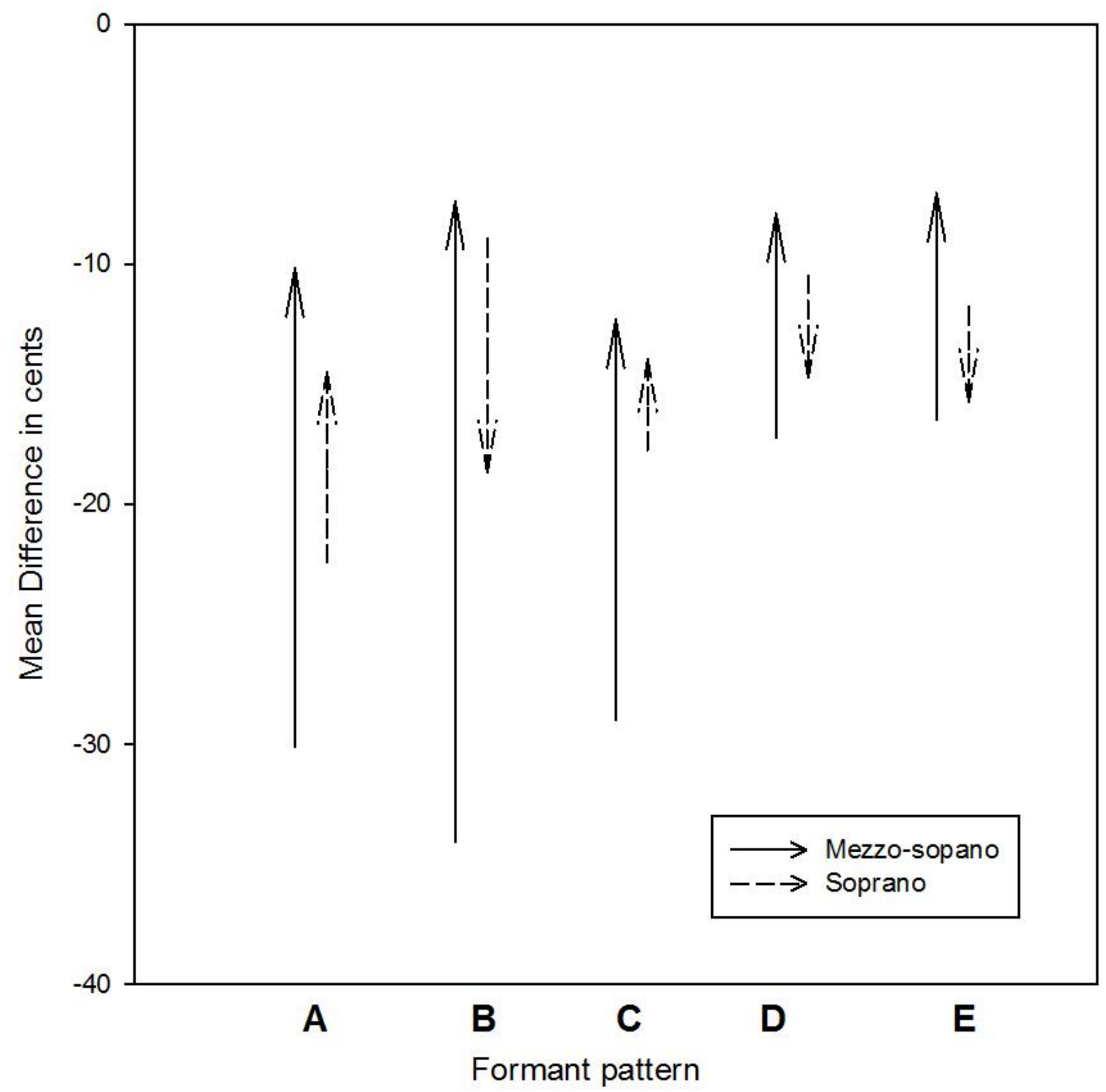

Figure 31. Vector plot representing mean difference in cents from pre-phonatory phase to mid-point of the vowel in vibrato condition at pitch B4. 


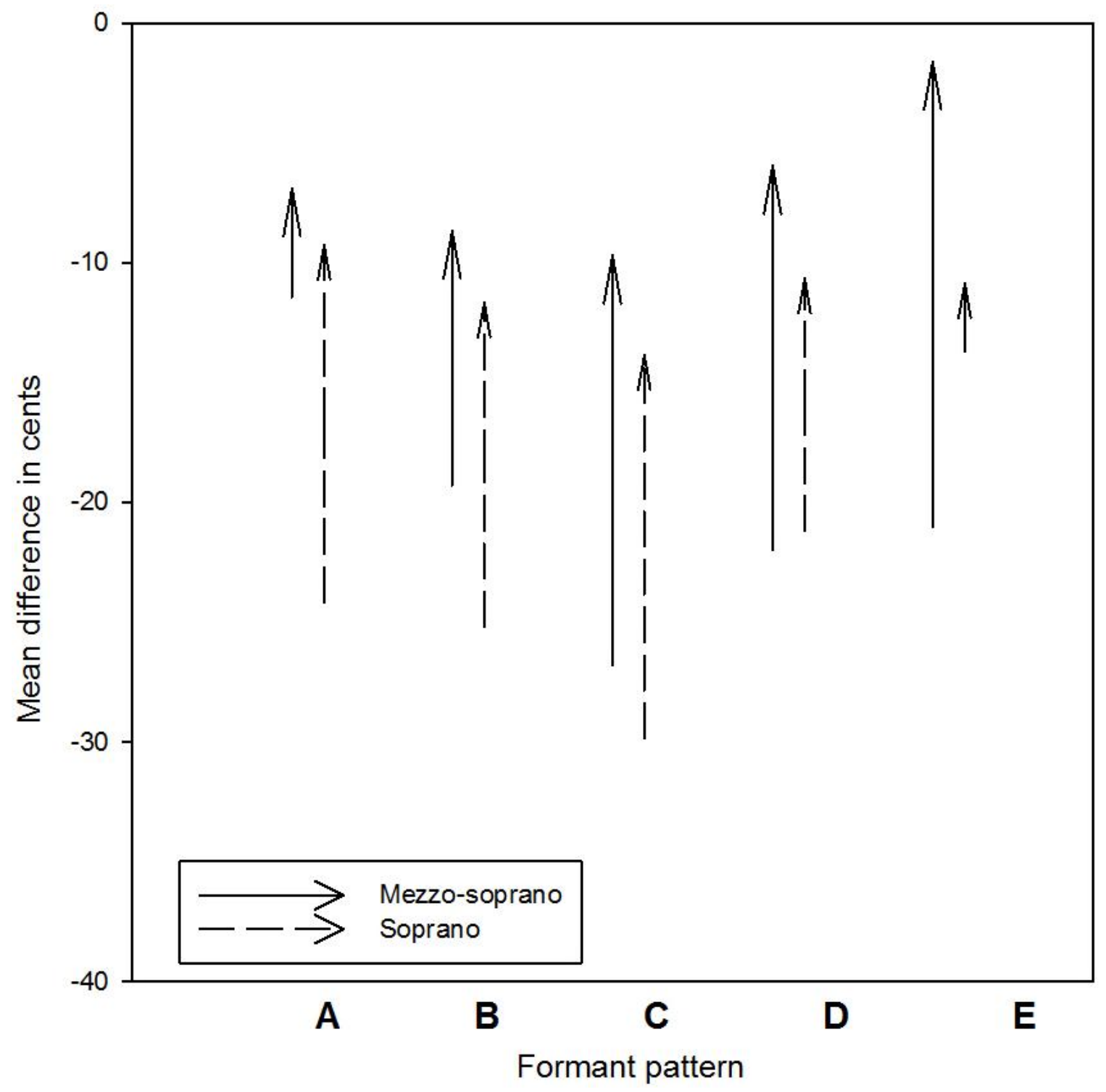

Figure 32. Vector plot representing mean difference in cents from pre-phonatory phase to mid-point of the vowel in vibrato condition at pitch F5. 


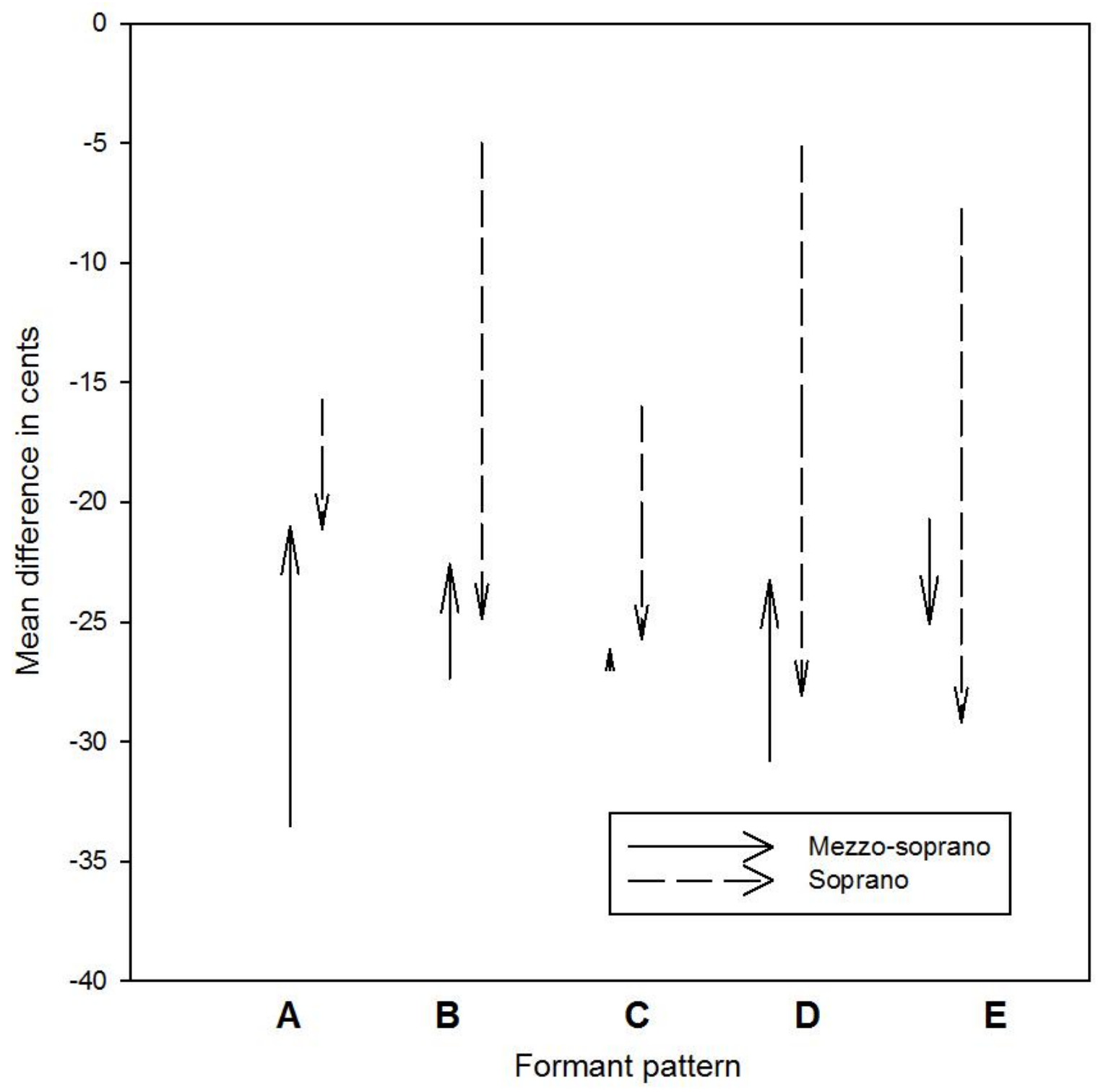

Figure 33. Vector plot representing mean difference in cents from pre-phonatory phase to mid-point of the vowel in no-vibrato condition at pitch $\mathrm{C} 4$. 


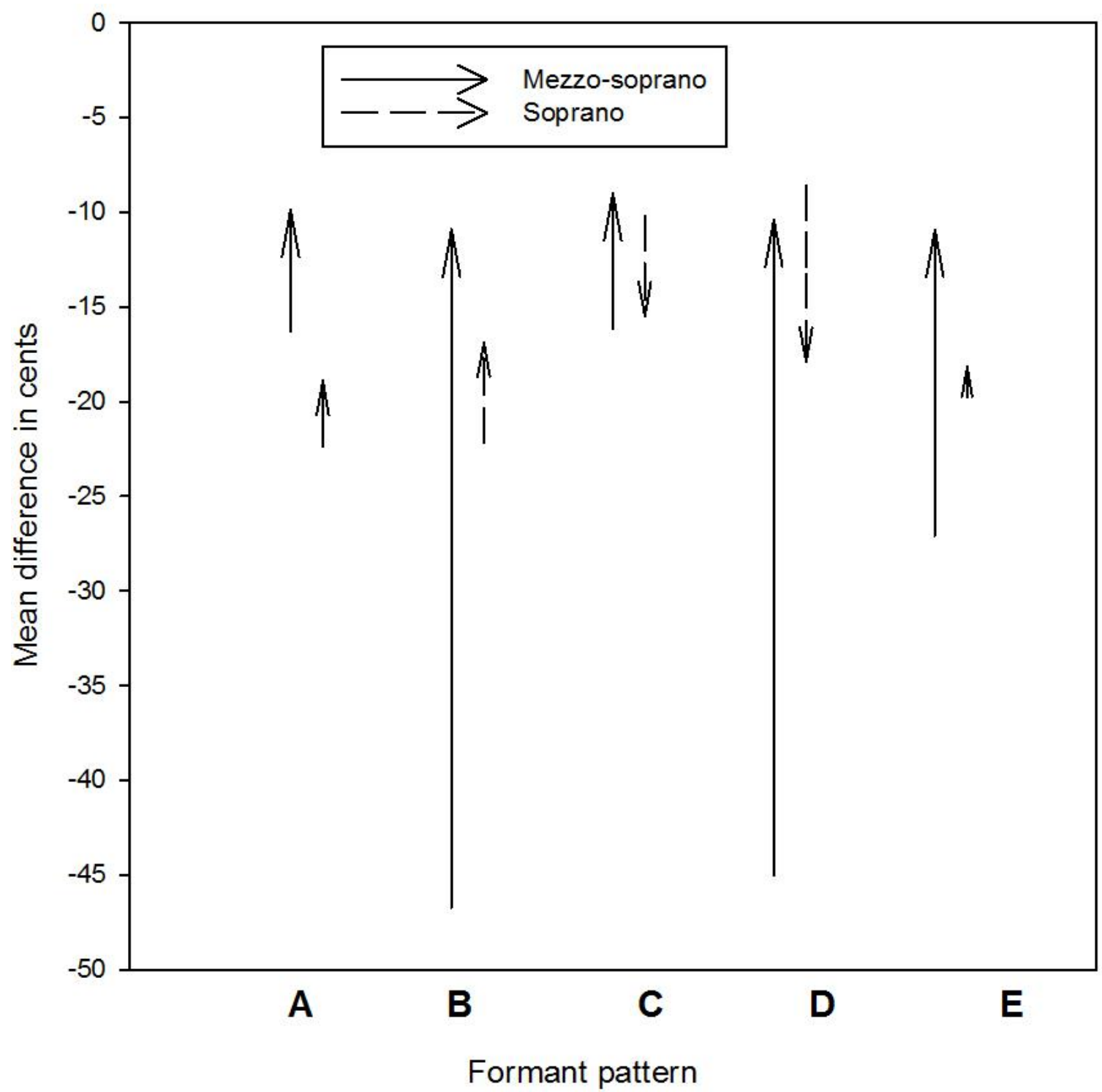

Figure 34. Vector plot representing mean difference in cents from pre-phonatory phase to mid-point of the vowel in no-vibrato condition at pitch B4. 


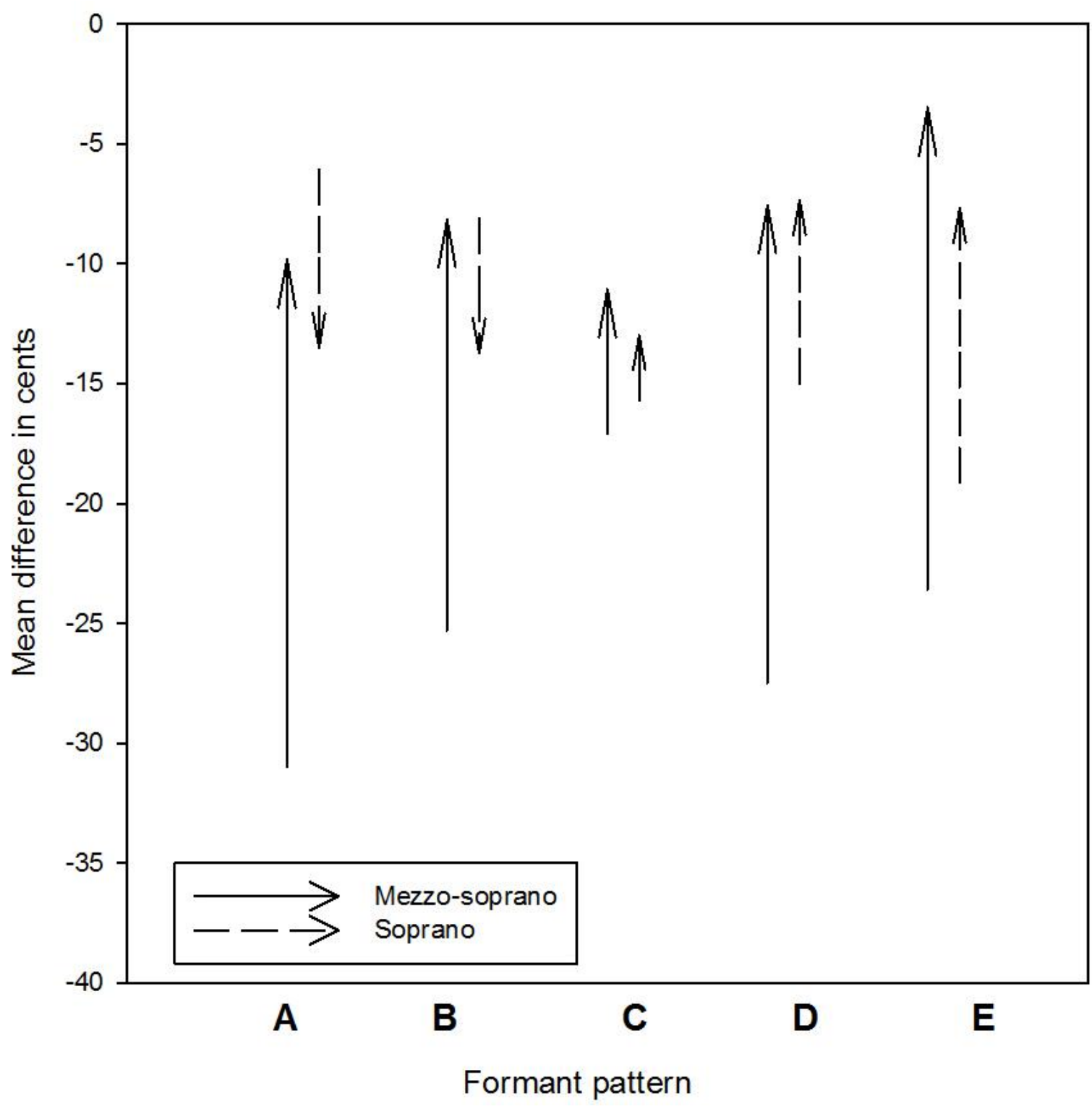

Figure 35. Vector plot representing mean difference in cents from pre-phonatory phase to mid-point of the vowel in no-vibrato condition at pitch F5. 


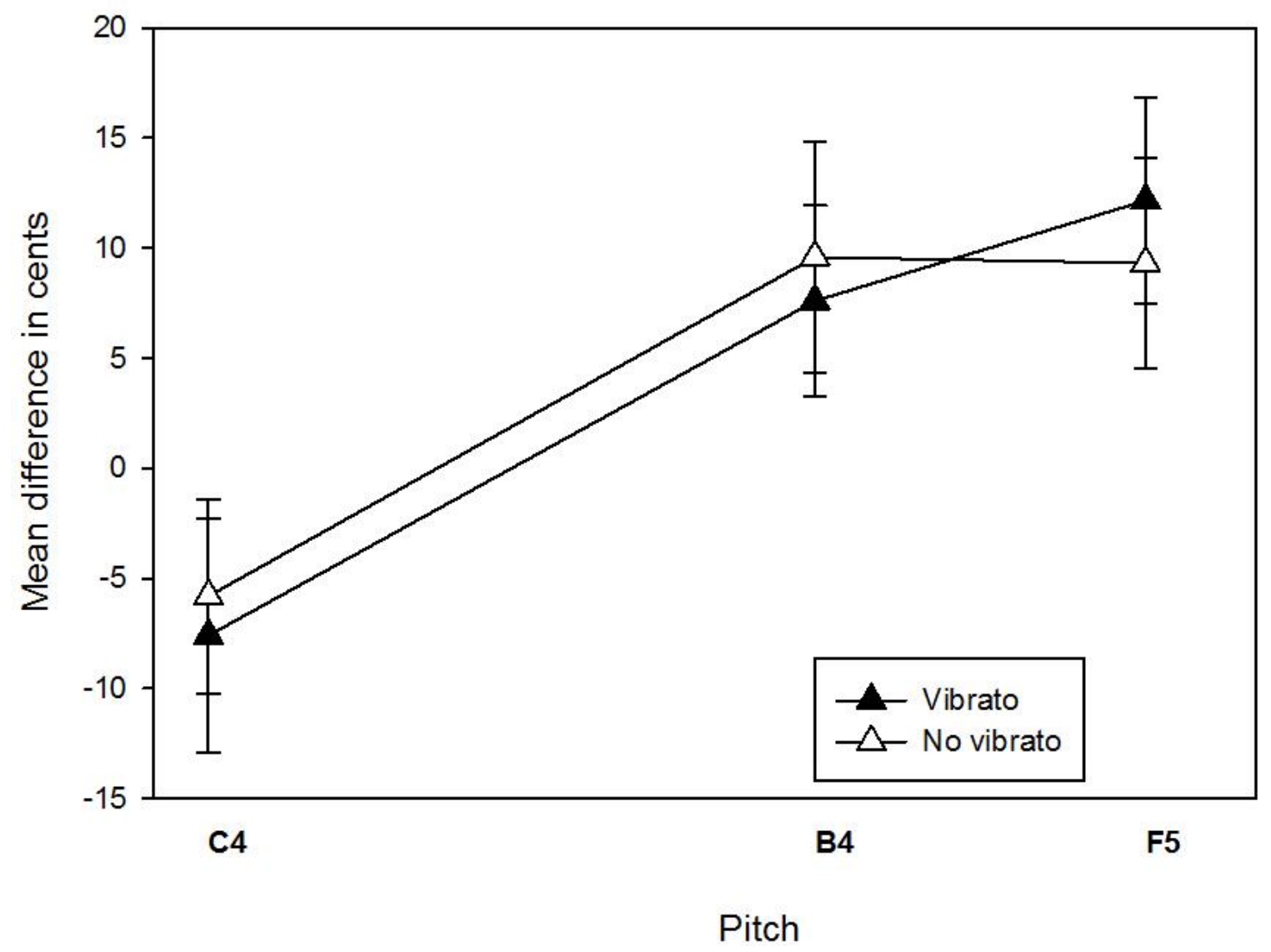

Figure 36. Mean difference in cents between pre-phonatory and mid-point of the vowel as a function of pitch for vibrato and no-vibrato conditions. 


\section{CHAPTER 5. DISCUSSION}

The present study investigated the effect of timbre and vibrato on pitch matching ability in western classical singers. Ten mezzo-sopranos and 12 sopranos with at least 3 years of training participated in the study. Singers were asked to match the pitch of synthetic stimuli that were synthesized with 5 different formant patterns (representing different timbres) and 2 vibrato conditions (vibrato and no-vibrato). Pitch matching ability was measured as the mean difference in cents between target stimuli and experimental productions at 2 points: (1) pre-phonatory set and (2) mid-point of the vowel. Also, the mean centroids of the mezzo-sopranos' and sopranos' productions were calculated.

\section{Effect of Timbre on Pitch Matching Accuracy}

The present study hypothesized that singers' pitch matching ability might be enhanced if the timbre of the stimuli was closer to their own vocal timbre. The results indicated that there was no significant effect of target formant pattern on pitch matching ability in the pre-phonatory or mid-point of the vowel conditions. This finding does not support the notion that singers exhibit increased pitch matching ability when timbre of the stimulus model is closer to their own voice. Rather, it was observed that the target formant pattern had a significant main effect on mean centroid values of mezzo-sopranos and sopranos in the vibrato condition at all pitches. This means that as the timbre of the more natural sounding vibrato target stimuli changed, the vocal timbre of the singer's productions also changed significantly. A significant trend towards higher centroid values from the formant pattern A to the formant pattern $\mathrm{E}$ in both mezzo-sopranos and sopranos was observed. Since we know that the formant frequency values of the target stimuli increase linearly from pattern A to pattern $\mathrm{E}$, we can infer that singers tried to match the timbre of the target stimuli in the vibrato condition by raising or lowering the larynx. Given that singers are hearing and attempting to match the timbre of target stimuli, the probability that they would alter their pitch in an attempt to match the spectral centroid becomes reduced. In fact there is no evidence that singers as a group attempted to use pitch as a mechanism to match the spectral centroids of the target stimuli in the vibrato condition.

On the other hand, singers did not attempt to match their vocal timbre to the timbre of target stimuli when the stimuli were the less natural no-vibrato stimuli. If pitch matching is more accurate when the target timbre is closer to the singer's own timbre and the singer is not altering their own timbre to match that of the target stimulus, then we might then expect singers' pitch matching ability to be influenced by timbre for this condition. Statistically this was not the case. Only at the pitch F5 for the no-vibrato condition do we observe that singers moved closer to being in tune as the formant pattern increased from $\mathrm{A}$ to $\mathrm{E}$ in a manner similar to the hypothetical situation presented in Figure 1. Had this finding been statistically significant, we would have expected a significant interaction between pitch and pattern for the no-vibrato condition. In fact, the 
interaction between pitch and pattern for the no-vibrato condition does trend toward significance and would have been so if it were not for the rigorous application of the Bonferroni correction. This trend could be a random effect or could turn out to be a true finding. Only by increasing the sample size can we further investigate this possibility.

\section{Effect of Target Pitch on the Pitch Matching Accuracy}

The present study hypothesized that pitch matching ability might vary with increasing pitch. In the context of the hypothesis that singers would be more likely to accurately match pitch when the target timbre is more like their own timbre, it was hypothesized that at high pitches, where harmonics are more widely spaced, such differences would not emerge since the exact locations of the formants would likely not be realized in the output spectrum, eliminating differences in spectral centroid as a function of pattern. However, analyses of the actual centroids of the target stimuli revealed that the differences in centroid between the most extreme patterns, A and E actually increase with increasing pitch. The difference in spectral centroid between pattern $\mathrm{A}$ and pattern $\mathrm{E}$ at the lowest pitch, $\mathrm{C} 4$, was 7 semitones, while this difference at the higher pitches, B4 and F5, was 11 semitones. Given this unexpected finding, we would expect pattern to be more of an effect at the higher pitches, B4 and F5.

Statistically, this should have resulted in a significant interaction between pitch and pattern, which did not occur. However, as mentioned in the previous section where the effects of timbre are discussed, there was a nearly significant interaction between pitch and pattern. As shown in that section, a pattern of responses consistent with the idea that singers more accurately match pitch when the target stimulus is more like their own timbre did emerge in the no-vibrato condition at F5, but not at B4. Examined in this context, further exploration of interaction of pitch and timbre on pitch matching would likely be warranted.

The present study did not hypothesize that there would be an overall main effect of target pitch on pitch matching ability, yet in the mid-point of the vowel condition, there was a main effect of target pitch, with pitch accuracy increasing as a function of the target pitch. With the application of Bonferroni corrections, there was a significant interaction with target pitch and vibrato, but no other significant interactions with pitch. In both the vibrato and no-vibrato conditions, pitch remained a significant main effect. This effect can be seen in Figure 18. Post-hoc contrast analyses indicated that for both vibrato and no-vibrato conditions, the trend is significant and quadratic. However, in Figure 18 the spacing between the pitches has been modified to reflect the actual distance between them in semitones. It can be seen that the relationship between pitch accuracy and target pitch is mostly linear for the no-vibrato stimuli and likely contains both a significant linear and quadratic component for the vibrato condition. This result was not expected.

Changes in pitch accuracy with target pitch could be predicted by two mechanisms (1) a physiological model based on vocal fold mechanics or (2) an auditory perceptual model. In a physiological model, we would expect difference in pitch 
matching accuracy from low to high pitches based on differential activation of the thyroarytenoid and cricothyroid muscles. At low pitches, the vocal folds must be shortened beyond their resting length through activation of the thyroarytenoid, resulting in decreased length, but also increased cross-sectional mass and decreased stiffness. At mid-range pitches, the vocal folds are closer to their resting length and less muscular effort is necessary to achieve the desired pitch. At high frequencies, the cricothyroid must be actively engage in order to lengthen, and more importantly, decrease the crosssectional mass and increase stiffness, to raise the fundamental frequency to the desired pitch. Based on a purely physiological mechanism, we would expect lower notes would tend to be sharp, mid-range notes would tend to be the most in tune, and high frequency notes would tend to be flat. We also would expect this effect to be the most pronounced during the pre-phonatory set condition. In fact, there was no significant effect of target pitch in the pre-phonatory condition and the significant effect at the mid-point of the vowel did not follow the pattern expected based on a purely physiological model.

Possible auditory perceptual mechanisms causing pitch accuracy to improve with increasing target pitch are difficult to determine. To better understand how the increase in pitch accuracy with target pitch might occurs at the mid-point of the vowel, it is necessary to discuss this process in the context of the difference between the prephonatory set and mid-vowel conditions. The general pattern of change in pitch accuracy from pre-phonatory to mid-vowel for vibrato and no-vibrato conditions at all three pitches is presented in Figures 30-35. Figure 37 shows the overall pattern of change in accuracy in pitch as a function of target pitch for mezzo-sopranos and sopranos, averaged across all patterns and vibrato conditions. It can clearly be seen that at pitch $\mathrm{C} 4$, regardless of the pitch at the pre-phonatory set, after feedback, both mezzo-sopranos and sopranos alter their fundamental frequency so that on average it is approximately 27 cents flat, well below the typical non-musician pure tone difference limen. Even more interestingly, on average the sopranos start off relatively in tune during the pre-phonatory set and very systematically lower their pitch flatter after feedback. It is also striking, that on average, both sopranos and mezzo-sopranos appear to be aiming for approximately the same, noticeably flat, frequency. The pattern of behavior for mezzo-sopranos and sopranos is less consistent at the higher pitches, B4 and F5. However, it appears that the target frequencies for both these groups increases with target pitch become more in tune with increasing frequency. The auditory perceptual mechanism for this phenomenon is not known.

\section{Effect of Vibrato on Pitch Matching Accuracy}

The present study hypothesized that pitch matching ability of adult classical singers would differ depending on whether or not the target stimulus was produced with vibrato or without vibrato. No significant effect of vibrato on pitch matching was seen in either the pre-phonatory or mid-vowel conditions. Neither the frequency modulation (FM) nor the coincident amplitude modulation (AM) caused by vibrato affected the pitch matching task in either mezzo-sopranos or sopranos. 
Pre-phonatory set to mid-point of the vowel

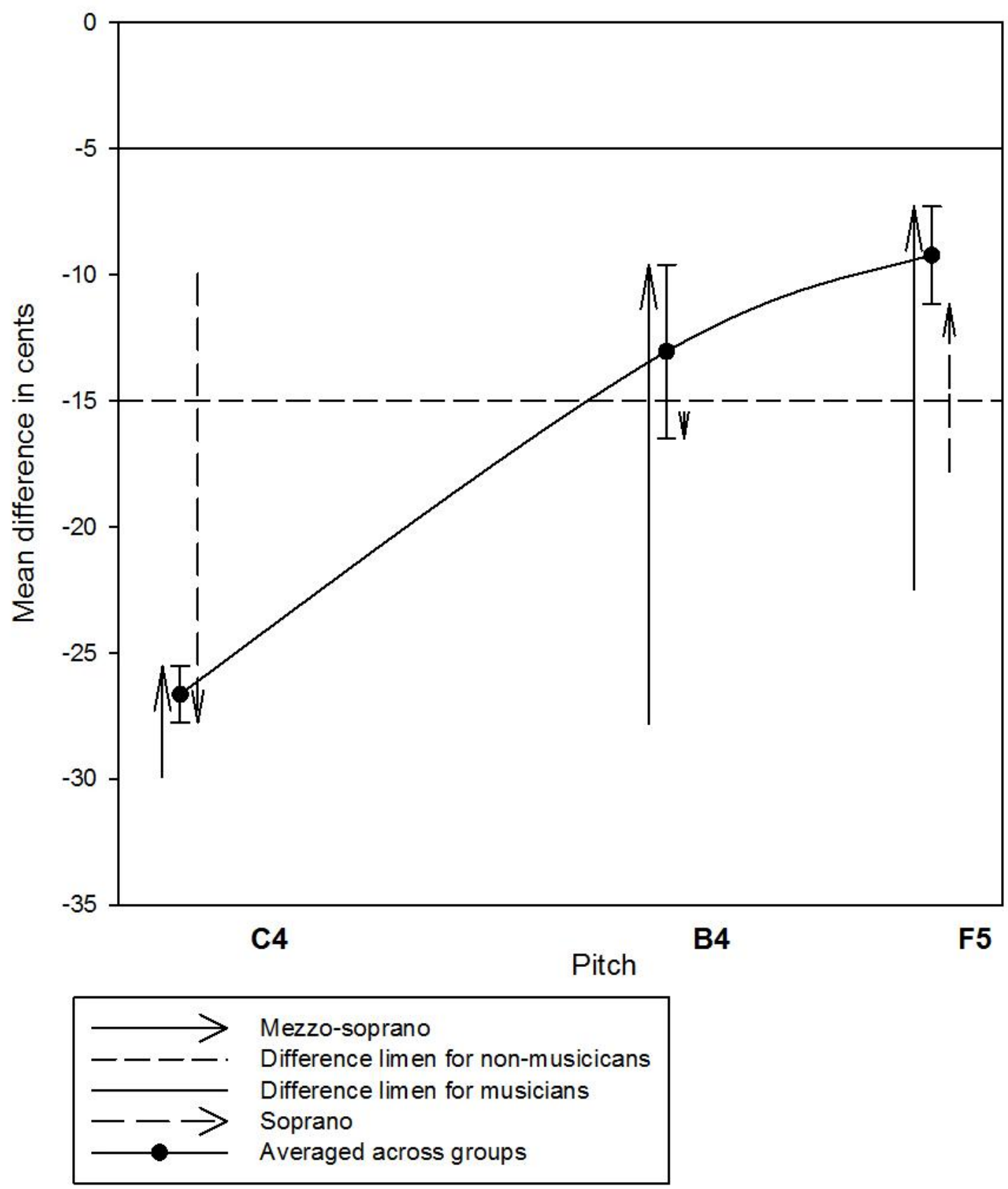

Figure 37. Change in pitch matching accuracy as a function of target pitch for mezzo-sopranos and sopranos averaged across all patterns and vibrato conditions. 
This is not entirely surprising based on the results of studies conducted by Sundberg $(1972,1978)$, Brown and Vaughn (1996) Yarbrough, Bowers \& Benson (1992). Using pure tones, Sundberg (1972) found no effect of a vibrato modulation at 6.5 $\mathrm{Hz}$ and an extent of $1.7 \%$. However, in his 1978 pure tone study, Sundberg did find that vibrato could affect the perception of pitch, but only for vibrato rates equal to or less than $4 \mathrm{~Hz}$. Brown and Vaughn also found no effect of vibrato on tuning tasks in experienced adult musicians tuning to a cello. Yarbrough, Bowers, and Benson showed that vibrato did not have any effect on the pitch matching tasks in 'certain' singers. However, in "uncertain" singers, vibrato affected the performance in the pitch matching tasks. In the current study, the participants are adults with years of vocal study who would not be defined as "uncertain" and the vibrato rate of $5.6 \mathrm{~Hz}$ was above the $4 \mathrm{~Hz}$ threshold of Sundberg's (1978) and closely located to the $6.5 \mathrm{~Hz}$ rate of his 1972 study. Thus, it is not surprising that vibrato was not a main effect in the study.

\section{Effect of Category on Pitch Matching Accuracy}

An alternative hypothesis proposed in this study was that mezzo-sopranos on average tend to sing less accurately and below the target pitch than sopranos. There are no published studies that document this phenomenon; however, it is a belief held by some singing experts. There are two main mechanisms that might be responsible for such a finding. The first one would be primarily a physiological mechanism. The vocal folds of mezzo-sopranos generally vibrate at lower frequencies. This implies that they are either (1) longer than those of sopranos, (2) thicker than those of sopranos, or less stiff than those of sopranos. For any given pitch above that produced when their cricothyroid muscle is at rest, mezzo-sopranos will have to engage greater muscle activity to decrease vocal fold thickness and increase vocal fold stiffness than will their soprano counterparts. One possible effect of this necessarily increased effort could be pitch undershoot, particularly at the pre-phonatory set.

The second possible explanation is an auditory-perceptual mechanism. In this case it may be that mezzo-sopranos and sopranos have different internal models of their own vocal timbre, based on the fact that sopranos have shorter vocal tract and higher formant frequencies than do mezzo-sopranos. When they attempt to produce a pitch, after the pre-phonatory phase, when they begin to process the feedback from their own voices, they may wish to accentuate the "mezzo-sopranoness" or "sopranoness" of their own voices by further lowering or raising their spectral centroids. The best way to do this would be for mezzo-sopranos to lower their larynx and for sopranos to raise their larynx. However, if the perception of timbre and pitch are not clearly separate, they may also attempt to alter the pitch, with mezzo-sopranos tending to lower their pitch and sopranos tending to raise it.

In the current study there was no significant effect of voice category on pitch matching ability in the pre-phonatory condition. This appears to rule out a physiological mechanism causing mezzo-sopranos to initiate phonation at a lower pitch. However, visual examination of the data in Figures 8-11 seems to suggest that the mezzo-sopranos 
did initiate phonation at lower frequencies than did sopranos at the pitches C4 and B4, but not at F5. Given that we might expect this phenomenon to increase with increasing pitch, which it does not, it may be that (1) the current findings are just random variation or (2) there are intervening factors that have not yet been accounted for in the pitch matching model. Based on the current study, however, it cannot be concluded that a physiological mechanism causes mezzo-sopranos to initiate phonation at a lower pitch.

Likewise, there was no significant main effect of voice category in the mid-point of the vowel condition in the current study. Neither are there trends that can be observed in that data that might suggest an effect that may emerge with a larger sample size. On average, all of the singers, regardless of voice category, even after processing feedback from their own voices, tend to sing at a frequency lower than the target pitch. Sometimes, as seen at the pitch $\mathrm{C} 4$, this effect exceeds the typical difference limens for nonmusicians and therefore would be quite perceivably lower.

However, there was a significant main effect of voice category when the difference in pitch matching accuracy between the pre-phonatory and mid-point of the vowel was the dependent variable. In Figure 38, the change in pitch accuracy between pre-phonatory and mid-vowel conditions is presented averaged over all pitches. Positive values indicate that on average, the singers increased their fundamental frequency from pre-phonatory to mid-vowel. Negative values indicate that on average, the singers decreased their fundamental frequency from pre-phonatory to mid-vowel. It should be noted that these values taken by themselves do not indicate how in tune the singers were at either the pre-phonatory or mid-vowel measurement, but simply show the magnitude and direction of the average change. Generally, mezzo-sopranos increased their fundamental frequency from pre-phonatory to mid-vowel, while sopranos either changed their frequency very little or on average increased their frequency from pre-phonatory to mid-vowel. Given that the frequencies of the mid-vowel condition were not significantly different for mezzo-sopranos and sopranos and also did not appear to show any trend in that direction, it must be a difference in the pre-phonatory set between these two voice categories that accounts for the significant difference in change of fundamental frequency from pre-phonatory to mid-vowel.

\section{The Effect of Target Timbre on Spectral Centroid}

This study did not present a hypothesis that the timbre of the target stimulus would affect the timbre of experimental productions. However, in order to determine whether or not the participants in the study exhibited the timbre difference typically associated with mezzo-sopranos and sopranos, an analysis of the differences in spectral centroid between the two groups was conducted. The analysis revealed that, indeed, mezzo-sopranos showed lower spectral centroids than did their soprano counterparts. This finding is well established in the literature. Erickson (2003) reported that the spectral centroid highly correlated with voice category in both mezzo-sopranos and sopranos. At low to mid frequencies, spectral centroid highly correlates with the higher formants F3 and $\mathrm{F} 4$ and is thus an indicator of vocal timbre. 


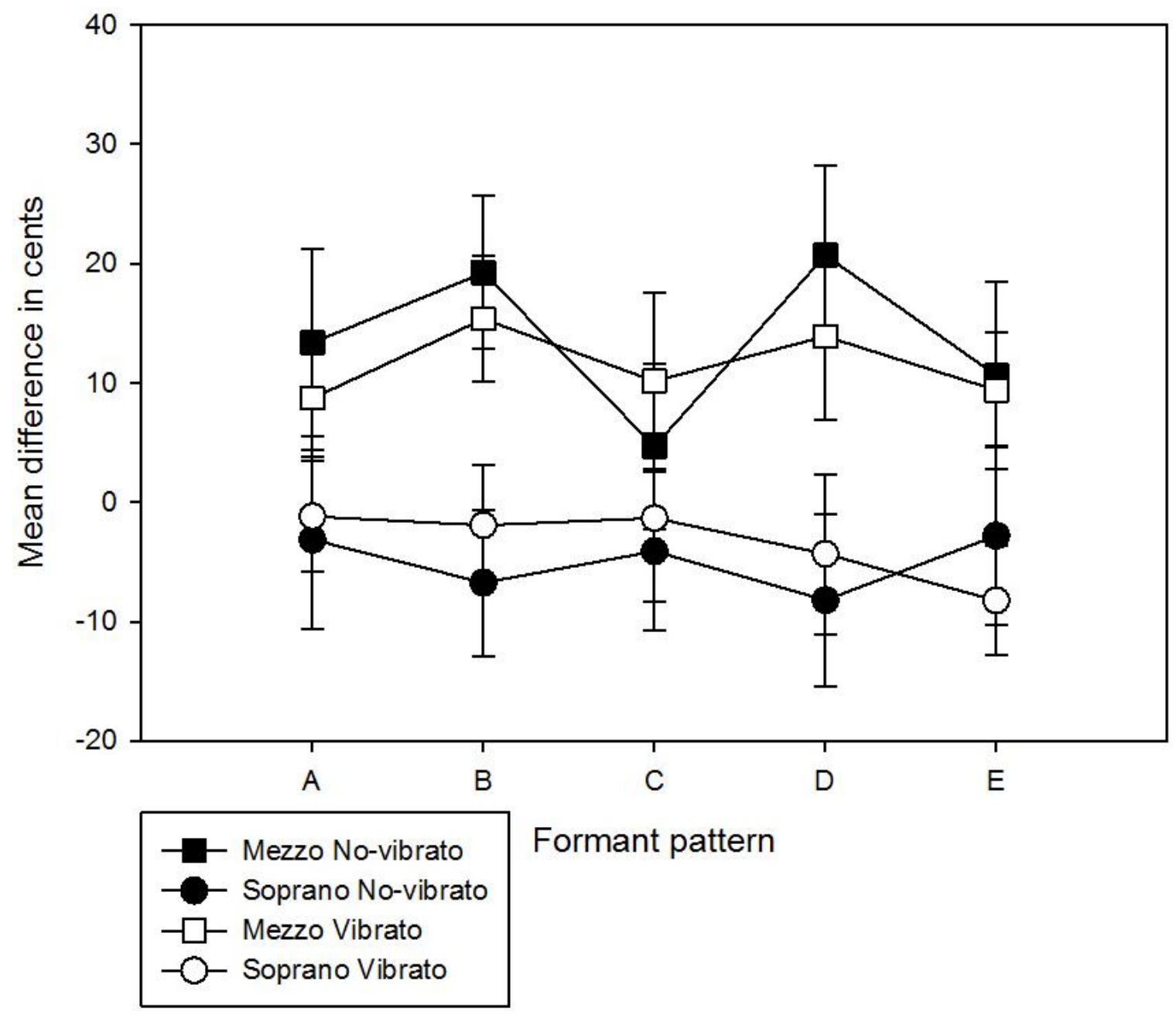

Figure 38. Change in pitch matching accuracy between pre-phonatory set to mid-point of the vowel averaged over all the pitches. 
Higher formant values for sopranos compared to mezzo-sopranos have also been found (Cleveland, 1977; Dmitriev and Kiselev, 1979; Iverson, et.al, 1993). Thus, the groups considered in the study were confirmed as true mezzo-sopranos and sopranos based on the centroid values.

While not hypothesized, it might be expected that singers alter their timbre based on the timbre of the model. The vocal tract is highly flexible and singers may alter their resonance frequencies by raising and lowering the larynx. There is a great deal of overlap between the possible vocal tract lengths of sopranos and mezzo-sopranos.

Target pattern was found to significantly affect spectral centroid in mezzosopranos and sopranos. However, this effect significantly interacted with vibrato. When the effect was analyzed separately for no-vibrato and vibrato conditions, target pattern was found to significantly affect spectral centroid in the vibrato condition only. For both mezzo-sopranos and sopranos, when the target stimulus was the more natural vibrato condition, as the formant pattern of the stimulus increased, on average, so did the singers' spectral centroids. This suggests that the singers raised the larynx to attempt to match the timbre of the target pitch only when vibrato was present. While it is unclear exactly why this would occur only in the vibrato condition, the vibrato condition is a much more natural sounding condition. Even though there are many singing styles in which singers often sing without vibrato, those natural productions in fact have small frequency and loudness variations that would not be present in the no-vibrato stimuli. It is likely that the vibrato stimuli were heard as being more like human voices and were the no-vibrato stimuli.

This tendency to attempt to match the timbre of human voices could be an innate characteristic of human beings or it could be a learned behavior. Trained singers regularly sing in vocal ensembles where blend is highly emphasized. In these ensembles, they are highly encouraged to match the timbre of other singers. Without a control group of individuals without musical or choral training, it is impossible to know determine the exact reason why the timbre matching to the more human-like stimuli occurred.

\section{Limitations of the Study}

While it is useful to see how factors interact in a model and in fact, it is dangerous to come to conclusions about main effects without examining how factors interact in a model, the inclusion of the number factors used in this study combined with the low number of subjects, makes it difficult to disentangle interactions. A larger number of subjects are needed and a more parsimonious model may be required.

Spectral centroid, while providing the most useful quantitative measure available for timbre, is not without problems. At higher pitches, when harmonics become widely spaced, changes in formant frequency may result in subtle spectral changes that do not result in a change in spectral centroid. It is likely that human beings hear these changes. Therefore, at higher pitches, differences in spectral centroid may not correlate with 
perceived differences in timbre. Future studies should include a perceptual task where listeners are presented with paired stimuli synthesized with differing formant patterns, some of which result in the calculation of different spectral centroid, some of which do not, and asked to rate how different in timbre the two stimuli are.

In attempting to determine whether or not frequency differences would be audible to the listener, average pure tone difference limens (DLs) were used. These DLs may not be appropriate for complex sung vocal stimuli. Future research should establish more precise DL values for complex vocal sounds at a variety of pitches.

The subjects in this study were highly trained vocal musicians. The results cannot be generalized to the general population of non-musicians.

\section{Summary and Conclusions}

It was hypothesized that the ability to accurately match pitch would be enhanced if the timbre of the target stimulus was closer to that of the singer. This was not found to be true. It was also hypothesized that based on the wider spacing of the harmonics at higher pitches, any effects of timbre on pitch accuracy might be reduced at higher pitch. This was also found not to be true. There was, however, an unexpected main effect of target pitch on pitch accuracy, with higher pitches being produced more accurately than lower pitches. The exact mechanism for this effect remains unknown. It was hypothesized that there may be an effect of vibrato on pitch accuracy. This was not true. Finally, it was hypothesized that in general mezzo-sopranos would be less accurate in a negative direction than sopranos. This was also not true. An unexpected finding was that singers attempted to match the timbre of the target stimuli in the vibrato condition only. It remains unclear whether or not that is due to the more naturally human-like quality of these stimuli, and if so, is this is a learned behavior or an innate human behavior.

The main hypothesis of this study, that the timbre of the target stimulus would affect pitch accuracy, was based on the concept of spectral pitch detection. It was believed that if spectral pitch processing was being utilized by the singers, it could result in a confusion of timbre and pitch. Given the current findings, these results do not provide clear evidence of the use of spectral processing. Thus, the type of processing, spectral or temporal, cannot be deduced from this study. 


\section{LIST OF REFERENCES}

American National Standards Institute (1973). American national psychoacoustical terminology. S3.20. New York: American Standards Association.

Amir, O., Amir, N., \& Kishon-Rabin, L. (2003). The effect of superior auditory skills on vocal accuracy. Journal of the Acoustical Society of America, 113(2), 1102-8.

Baddeley, A.D., \& Hitch, G.J.L (1974). Working Memory, In G.A. Bower (Ed.), The psychology of learning and motivation: advances in research and theory (Vol. 8, pp. 47-89), New York: Academic Press.

Beal, A.L. (1985). The skill of recognizing the musical structures. Memory \& cognition, $13,405-412$.

Berndtsson, G, and Sundberg, J. (1995). Perceptual significance of the center frequency of the singer's formant. Scand J Logop Phonatr, 20, 35-41.

Boring, E. G. (1942) Sensation and perception in the history of experimental psychology. New York: Appleton-Century.

Clegg, B. (1966). A comparative study of primary grade children's ability to match tones. Unpublished master's thesis, Brigham Young University, Provo, UT.

Cleveland, T. F. (1977). Acoustic properties of voice timbre types and their influence on voice classification. Journal of the Acoustical Society of America, 61, 1622-1629.

Crowder, R. G. (1989). Imagery for musical timbre. Journal of Experimental Psychology: Human Perception and Performance, 15, pp. 472-478.

de Boer, D. (1976). On the "residue" and auditory pitch perception. In Keidel W. D. \& Neff, W. D. (Eds) Handbook of sensory physiology, vol V-3. (pp. 479-583). Berlin: Springer-Verlag.

de Cheveigné, A. (2005). Pitch perception models. In Plack, C.J. Oxenham, A. Fay, R.R. Popper, A.N. (Eds) Pitch - Neural coding and perception. (pp. 169-233). New York: Springer.

Demorest, S. M. (2001). Pitch-matching performance of junior high boys: A comparison of perception and production. Bulletin of the Council for Research in Music Education, 151, 63-70.

Dmitriev, L, and Kiselev. A. (1979). Relationship between the formant structure of different types of singing voices and the dimensions of the supraglottic cavities. Folia Phoniatrica (Basel), 31, 238-241. 
Doughty, J. M., and Garner, W. R. (1947). Pitch characteristics of short tones. I. Two kinds of pitch threshold. J Exp Psychol, 37, 351-365.

Doughty, J. \& Garner, W. (1948). Pitch characteristics of short tones II: Pitch as a function of tonal duration, Journal of Experimental Psychology, 38, 478-494.

Elliot, L., and Niemeoller, A. (1970). Role of hearing in voice fundamental frequency, International Audiology, 9, 47-52.

Elman, J. (1981). Effects of frequency-shifted feedback on the pitch of vocal production, J. Acoust. Soc. Am. 70, 45-50.

Erickson, M. L. (2004). The interaction of formant frequency and pitch in the perception of voice category and jaw opening in female singers. Journal of Voice, 18(1), 2437.

Fairbanks, G. (1955). Selective vocal effects of delayed auditory feedback. Journal of Speech and Hearing Disorders, 20 (4): 333-46.

Gaab, N. Gaser, C., Zaehle, T., Jancke, L., \& Schlaug, G. (2003). Functional anatomy of pitch memory - an fMRI study with sparse temporal sampling. NeuroImage, 19, 1417-1426.

Gaab, N. \& Schlaug, G. (2003). The effect of musicianship on pitch memory in performance matched groups. NeuroReport 14: 2291-2295.

Gelfand, S. A. (1998). Hearing: an introduction to psychological and physiological acoustics. Newyork, Marcel Decker.

Geringer, J. M. (1983). The relationship or pitch-matching and pitch discrimination abilities of preschool and fourth-grade students. Journal of Research in Music Education, 31, 93-99.

Goetze, M., Cooper, W., and Brown, C. (1990). Recent research in singing in the general music classroom, Bull. Council Res. Music Ed. 104, 16- 37.

Goldstein, J. L. (1973). An optimum processor theory for the central formation of the pitch of complex tones. J Acoust Soc Am, 54, 1496-1516.

Green, G. A. (1990). The effect of vocal modeling on pitch-matching accuracy of elementary schoolchildren. Journal of Research in Music Education, 38, 225-231.

Green, G. A. (1993). Unison versus individual singing and elementary students' vocal pitch accuracy. Journal of Research in Music Education, 42(2), 105-114. 
Grimault, N., Micheyl, C., Carlyon, R. P., and Collet, L. 2002 . "Evidence for two pitch encoding mechanisms using a selective auditory training paradigm," Percept. Psychophys. 64, 189-197.

Hain, T., Burnett, T., Kiran, S., Larson, C., Singh, S., and Kenney, M. (2000). Instructing subjects to make a voluntary response reveals the presence of two components to the audio-vocal reflex, Exp. Brain Res.130, 133-141.

Hartmann, W.M., Rakerd, B., and Packard, T.N. (1985). On measuring the frequency difference limen for short tones. Perception \& Psychophysics, 38, 199-207.

Helmholtz, H. L. F. (1954). On the Sensations of Tone as a Physiological Basis for the Theory of Music. New York: Dover. English translation of 1863 (German) edition by A. J.Ellis.

Hermanson, L. W. (1972). An investigation of the effects of timbre on simultaneous vocal pitch acuity of young children (Doctoral dissertation, Teachers College, Columbia University, 1971). Dissertation Abstracts International, 32, 3558A.

Houtsma, A. J. M. (1997). Pitch and Timbre: Definition, Meaning and Use. Journal of New Music Research 26, 104-115.

Johnson, D. H. (1980). The relationship between spike rate and synchrony in responses of auditory-nerve fibers to single tones. J Acoustic Soc Am, 68, 1115-1122.

Jones, J., and Munhall, K. (2000). Perceptual calibration of F0 production: Evidence from feedback perturbation, J. Acoust. Soc. Am. 108, 1246- 1251.

Jones, M. (1979). Using a vertical keyboard instrument with the uncertain singer. Journal of Research in Music Education, 27, 173-184.

Klemish, J. (1974). Treating the uncertain singer through the use of the tape recorder. Bulletin of the Council for Research in Music Education, 37, 36-45.

Leonard, R, \& Ringel, R. (1979). Vocal shadowing under conditions of normal and altered laryngeal sensation. J Speech Hear Res. 22, 794-817.

Lévêque. Y., Giovanni. A., Schön. D. (2011). Pitch-matching in poor singers: Human model advantage, Article in press, Journal of Voice.

Leydon, C., Bauer, J., and Larson, C. (2003). The role of auditory feedback in sustaining vocal vibrato, J. Acoust. Soc. Am. 114, 1575-1581.

Licklider, J. C. R. (1951). A duplex theory of pitch perception. Experientia 7(4), 128134. 
Moore, B.C.J. and Glasberg, B.R. (1990). Frequency discrimination of complex tones with overlapping and non-overlapping harmonics, J. Acoust. Soc. Am. 87: 21632177.

Moore B.C.J, Glasberg, B.R. and Proctor, G. M. (1992). Accuracy of pitch matching for pure tones and complex tones with overlapping and non-overlapping harmonics. $J$ Acoust Soc Am, 91, 3443-3450.

Murry, T. (1990). Pitch-matching accuracy in singers and non-singers. Journal of Voice, 4, 317-321.

Patterson, R. D. (1990). The tone height of multiharmonic sounds. Music Perception, 8, 203-214.

Pederson, D.M. \& Pederson, N.O. (1970). The relationship between pitch recognition and vocal pitch production in sixth-grade students. J Res Music Ed.18, 265-272.

Perry, D., Zatorre, R., Petrides, M., Alivisatos, B., Meyer, E., and Evans, A. (1999). Localization of cerebral activity during simple singing, NeuroReport 10(18), 3979-3984.

Petzold, R. G. (1966). Auditory perception of musical sounds by children in the first six grades. (Cooperative Research Project 1051). Madison, WI: University of Wisconsin-Madison.

Platt, J., \& Racine, R. (1985). Effect of frequency, timbre, experience and feedback on musical tuning skills. Perception and Psychophysics, 38 (6), 543-553.

Plomp, R. (1967). Pitch of complex tones. H Acoust Soc Am, 41, 1526-1533.

Ritsma, R. J. (1967). Frequencies dominant in the perception of the pitch of complex tones. $J$ Acoust Soc Am, 42, 191-198.

Russo, F. A., \& Thompson, W. F. (2005). An interval-size illusion: The influence of timbre on the perceived size of melodic intervals. Perception \& Psychophysics, $67,559-568$.

Schouten, J. F. (1938). The perception of subjective tones in Psychological Acoustics. Edited by E.D. Schubert, 1979.

Schouten, J. F. (1940). The residue, a new component in subject sound analysis. Proc Kon Acad Wetensch (Netherlands), 43, 356-356.

Schubert, E., Wolfe, J. and Tarnopolsky, A. (2004) Spectral centroid and timbre in complex, multiple instrumental textures, in Proceedings of the International Conference on Music Perception and Cognition, Evanston, Illinois. 
Shamma, S. \& Klein, D. (2000). The case of the missing pitch templates: How harmonic templates emerge in the early auditory system. J Acoust Soc Am, 107, 2631-2644.

Sims, W. L., Moore, R. A., \& Kuhn, T. L. (1982). Effects of male and female vocal stimuli, tonal pattern length, and age on vocal pitch-matching abilities of young children from England and the United States. Psychology of Music, Special Issue, 104-108.

Singh, P. G., \& Hirsh, I. J. (1992). Influence of spectral locus and F0 changes on the pitch and timbre of complex tones. Journal of the Acoustical Society of America, 92, 2650-2661.

Small, A. R. \& McCachern, F. L. (1983). The effect of male and female vocal modeling on pitch-matching accuracy of first-grade children. Journal of Research in Music Education, 31, 227-233.

Smith, E. E., \& Kosslyn, S. M. (2008) Cognitive Psychology: Mind and Brain. Saddle River, NJ: Pearson Prentice Hall.

Stauffer, S. L. (1985). An investigation of the effects of melodic and harmonic context on the development of singing ability in primary grade children. Dissertation Abstracts International, 46, 1862 A.

Stevens, S. S. (1935). The relation of pitch to intensity. J. acoust. Soc. Amer. 6, 150-154.

Stevens, S. S. (1975). Psychophysics: Introduction to its perceptual, neural, and social prospects (G. Stevens, Ed.). New York: Wiley.

Sundberg, J. (1972). Pitch of synthetic sung vowels. Department for Speech, Music, and Hearing Quarterly Progress and Status Report, 13, 34-44.

Sundberg, J. (1973). The source spectrum in professional singing. Folia Phoniatrica, 25, 71-90.

Sundberg, J. (1977). Effect of vibrato and singing formants on pitch, Journal of Research in Singing, 5, 5-17.

Sundberg, J. (1994). Perceptual aspects of singing. Journal of Voice, 8, 106-122.

Terhardt, E. (1974). Pitch, consonance, and harmony. Journal of the Acoustical Society of America 55(5), 1061-1069.

Terhardt, E. (1974). Pitch of pure tones: Its relation to intensity. In E. Zwicker \& E. Terhardt (Eds.), Facts and models in hearing (pp. 353- 360). Heidelberg: Springer-Verlag. 
Tenrhardt. E., Stoll. G., and Seewann. M (1982). Algorithms for extraction of pitch and pitch salience from complex tone signals. J Acoust Soc Am, 71, 679-688.

Titze, I. R. (1994). Principle of Voice Production. Englewood Cliffs, NJ: Prentice Hall.

Verschure, J., \& van Meeteren, A. A. (1975). The effect of intensity on pitch. Acoustica, $32,33-44$.

Ward, D., and Burns, E. (1978). Singing without auditory feedback, J.Res. Singing, 1, 24-44.

Watts, C., Barnes-Burroughs, K., Adrianopoulos, M., \& Carr, M. (2003). Potential factors related to untrained singing talent: A survey of singing pedagogues. Journal of Voice, 17, 298-307.

Watts, C., \& Hall, M. (2008). Timbral influences on vocal pitch-matching accuracy. Logopedics Phoniatrics Vocology, 33(2), 74-82.

Watts, C., Moore, R., \& McCaghren, K. (2005). The relationship between vocal pitchmatching skills and pitch discrimination kills in untrained accurate and inaccurate singers. Journal of Voice, 19, 534-543.

Weiner, 1. B., Lee, L., Cataland, J., \& Stemple, J. C. (1996). An assessment of pitchmatching abilities among speech language pathology graduate students, American Journal of Speech-Language Pathology, 5, 91-95.

Wightman, F. L. (1973). The pattern-transformation model of pitch. Journal of the Acoustical Society of America 54(2), 407-416.

Wilson, D. S. (1971). A study of the child voice from six to twelve. Dissertation Abstracts International, 31, $5453 \mathrm{~A}$.

Worthy, M. (2000). Effects of tone quality conditions on perception and performance of pitch among selected wind instrumentalists. Journal of Research in Music Education, 48 (3), 222-236.

Yarbrough, C., Bowers, J., \& Benson, W. (1992). The effect of vibrato on the pitch matching accuracy of certain and uncertain singers. Journal of Research in Music Education, 40, 30-38.

Yarbrough, C., Green G., Benson, W, \& Bowers, J. (1991). Inaccurate singers: An exploratory study of variables affecting pitch-matching. Bulletin of the Council for Research in Music Education, 107, 23-34. 
Yarbrough, C., Karrick, B., \& Morrison, S. J. (1995), Effect of knowledge of directional mistunings on the tuning accuracy of beginning and intermediate wind players. Journal of Research in Music Education, 43, 232-241.

Yarbrough, C, Morrison, S. J., \& Karrick, B. (1997). The effect of experience, private instruction, and knowledge of directional mistunings on the tuning performance and perception of high school wind players. Bulletin of the Council for Research in Music Education, 134, 31-42.

Zatorre, R. J., Evans, A. C., \& Meyer, E. (1994). Neural mechanisms underlying melodic perception and memory for pitch. The Journal of Neuroscience, 14, 1908-1919.

Zatorre, R. J., Evans, A. C., Meyer, E., \& Gjedde, A. (1992). Lateralization of phonetic and pitch processing in speech perception. Science, 256, 846-849. 


\section{VITA}

Sirisha Duvvuru was born in 1986 in India. She received her bachelor's degree

from All India Institute of Speech and Hearing in 2007. She was enrolled at The University of Tennessee into doctoral program in Speech and Hearing Science in August 2007. The author is a member of American Speech and Hearing Association. She received her doctorate in December 2012. 